\title{
Evolutionary Stages and Disk Properties of Young Stellar Objects in the Perseus Cloud
}

\author{
Hong-Xin Zhang ${ }^{\star \star 1,2,3,4,10}$, Yu Gao ${ }^{5,6}$, Min Fang ${ }^{5,7}$, Hai-Bo Yuan ${ }^{3}$, Yinghe Zhao ${ }^{5,6,8}$, \\ Ruixiang Chang ${ }^{9}$, Xuejian Jiang ${ }^{5,6}$, Xiao-Wei Liu ${ }^{3}$, A-Li Luo ${ }^{1}$, Hongjun Ma ${ }^{5,6}$, Zhengyi \\ Shao ${ }^{9}$ and Xiaolong Wang ${ }^{5,6}$ \\ 1 National Astronomical Observatories, Chinese Academy of Sciences, Beijing 100012, China \\ 2 Department of Astronomy, Peking University, Beijing 100871, China; hongxin@pku.edu.cn \\ ${ }^{3}$ Kevli Institute for Astronomy and Astrophysics, Peking University, Beijing 100871, China \\ ${ }^{4}$ Chinese Academy of Sciences South America Center for Astronomy, Camino EI Observatorio \\ \#1515, Las Condes, Santiago, Chile \\ 5 Purple Mountain Observatory, Chinese Academy of Sciences, Nanjing 210008, China; \\ yugao@pmo.ac.cn \\ 6 Key Laboratory of Radio Astronomy, Chinese Academy of Sciences, Nanjing 210008, China \\ 7 Departamento de Física Teórica Universidad Autónoma de Madrid, 28049 Cantoblanco, Madrid, \\ Spain; mfang.cn@gmail.com \\ 8 Infrared Processing and Analysis Center, California Institute of Technology, MS 100-22, Pasadena, \\ CA 91125 , USA \\ 9 Key Laboratory for Research in Galaxies and Cosmology, Shanghai Astronomical Observatory, \\ Chinese Academy of Sciences, Shanghai 200030, China \\ 10 Current address: Instituto de Astrofísica, Facultad de Física, Pontificia Universidad Católica de \\ Chile, Av. Vicuña Mackenna 4860, 7820436 Macul, Santiago, Chile
}

\begin{abstract}
We investigated the evolutionary stages and disk properties of 211 Young stellar objects (YSOs) across the Perseus cloud by modeling the broadband optical to midinfrared (IR) spectral energy distribution (SED). Our optical gri photometry data were obtained from the recently finished Purple Mountain Observatory (PMO) Xuyi Schmidt Telescope Photometric Survey of the Galactic Anti-center (XSTPS-GAC). About $81 \%$ of our sample fall into the Stage II phase which is characterized by having optically thick disks, and $14 \%$ into the Stage I phase characterized by having significant infalling envelopes, and the remaining 5\% into the Stage III phase characterized by having optically thin disks. The median stellar age and mass of the Perseus YSOs are 3.1 Myr and 0.3 $M_{\odot}$ respectively. By exploring the relationships among the turnoff wave bands $\lambda_{\text {turnoff }}$ (longward of which significant IR excesses above the stellar photosphere are observed), the excess spectral index $\alpha_{\text {excess }}$ as determined for $\lambda>\lambda_{\text {turnoff }}$, and the disk inner radius $R_{\text {in }}$ (determined from SED modeling) for YSOs of different evolutionary stages, we found that the median and standard deviation of $\alpha_{\text {excess }}$ of the YSOs with optically thick disks tend to increase with $\lambda_{\text {turnoff }}$, especially at $\lambda_{\text {turnoff }} \geq 5.8 \mu \mathrm{m}$, whereas the median fractional dust luminosities $L_{\text {dust }} / L_{\star}$ tend to decrease with increasing $\lambda_{\text {turnoff. }}$. This points to an inside-out disk clearing of small dust grains. Moreover, a positive correlation between $\alpha_{\text {excess }}$ and $R_{\text {in }}$ was found at $\alpha_{\text {excess }} \gtrsim 0$ and $R_{\text {in }} \gtrsim 10 \times$ the dust sublimation radius $R_{\text {sub }}$, irrespective of $\lambda_{\text {turnoff }}, L_{\text {dust }} / L_{\star}$ and disk flaring. This suggests that the outer disk flaring either does not evolve synchronously with the inside-out disk clearing of small dust
\end{abstract}

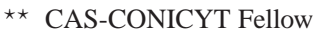


grains or has little appreciable influence on the spectral slopes at $\lambda \lesssim 24 \mu \mathrm{m}$. About $23 \%$ of our YSO disks are classified as transitional disks, which have $\lambda_{\text {turnoff }} \geq 5.8 \mu \mathrm{m}$ and $L_{\text {dust }} / L_{\star}>10^{-3}$. The transitional disks and full disks occupy distinctly different regions on the $L_{\text {dust }} / L_{\star}$ vs. $\alpha_{\text {excess }}$ diagram. Taking $L_{\text {dust }} / L_{\star}$ as an approximate discriminator of disks with $(>0.1)$ and without $(<0.1)$ considerable accretion activity, we found that $65 \%$ and $35 \%$ of the transitional disks may be consistent with being dominantly cleared by photoevaporation and dynamical interaction with giant planets respectively. None of our transitional disks have $\alpha_{\text {excess }}(<0.0)$ and $L_{\text {dust }} / L_{\star}(>0.1)$ values that would otherwise be suggestive of disk clearing dominantly by grain growth.

Key words: stars: formation - stars: low-mass - stars: pre-main sequence - individual: Perseus Cloud - circumstellar matter - protoplanetary

\section{INTRODUCTION}

The formation and early evolution of stars are among the central problems in Astrophysics. Young stellar objects (YSOs), which are primarily identified as pre-main-sequence (PMS) stars by the presence of infrared (IR) excess arising from circumstellar disks or surrounding envelopes (e.g. Allen et al. 2004; Greene et al. 1994; Lada 1987), have been extensively studied in nearby star-forming regions (e.g. Taurus: Luhman et al. 2010; NGC 1333: Winston et al. 2010; IC 348: Muench et al. 2007; $\sigma$ Ori: Hernández et al. 2007; Tr 37: Sicilia-Aguilar et al. 2006; NGC2362: Currie et al. 2009; Lynds 1630N, 1641: Fang et al. 2009, 2013). Studying the circumstellar environment, either disks or envelopes, around YSOs of different mass is essential to understanding the formation of both stars and their planetary systems.

YSOs are traditionally categorized into four classes or evolutionary stages based on the spectral index $\alpha(d \log (\lambda F(\lambda)) / d \log (\lambda))$ of their near- to mid-IR spectral energy distributions (SEDs; e.g. André et al. 1993; Greene et al. 1994; Lada 1987). The youngest Class 0 objects are only visible in far-IR to submm wavelengths, and they are thought to have envelope mass that exceeds the central stellar mass; Class I YSOs $(\alpha \geq 0.3)$ are characterized by rising mid-IR SEDs, and may be still in an envelope collapse stage but have the central stellar mass exceeding the envelope mass; Class II YSOs $(-1.6 \leq$ $\alpha<-0.3$ ) have SEDs that peak at near-IR wavelengths, decrease at longer wavelengths that is much more gradual than what expected for a stellar photosphere, and they agree well with PMS stars with circumstellar accretion disks; Class III YSOs $(\alpha<-1.6)$ have little or no IR excess, and are thought to be in the disk dissipation stage with very little or no circumstellar material. In addition, Greene et al. (1994) introduced an additional FLAT-spectrum class $(-0.3 \leq \alpha<0.3)$ which has spectral indices in between Classes I and II.

The star formation process is generally accompanied by the formation, evolution and dispersal of circumstellar protoplanetary disks which are believed to be the sites of planet formation. In particular, optically thick full disks are usually found in the Class II YSOs, whereas the evolved or anemic optically thin disks are usually identified with the Class III YSOs. A lot of important information about the evolutionary stages and disk properties of YSOs is encoded in the multi-wavelength SEDs (e.g. Robitaille et al. 2006, hereafter R06). For instance, the optical to near-IR bands offer important constraints on the properties of the central source, such as the temperature and bolometric luminosity; the near- to mid-IR bands provide a crucial constraint on the inner (from a few AU to tens of AU) disk properties; the far-IR to submm bands give strong constraints on the mass of disks and envelopes (e.g. Andrews \& Williams 2005).

As currently the most active site of low- to intermediate-mass star formation within $\sim 300$ pc of the Sun, the Perseus cloud ( $M \simeq 4.8 \times 10^{3} M_{\odot}$; Evans et al. 2009) region is an ideal laboratory for studying the formation and early evolution of low- to intermediate-mass stars (e.g. Bally et al. 2008) and the circumstellar disks. Recently, the Spitzer telescope observations, especially through the "Cores to Disks" legacy project (c2d; Evans et al. 2003), have led to the identification of over 400 YSOs (mostly 
Classes I and II) toward the Perseus cloud. In addition, systematic submm continuum surveys of the Perseus region with SCUBA (Hatchell et al. 2005) and Bolocam/CSO (Enoch et al. 2006) have led to the confirmation of over 100 protostellar or starless submm cores, and about one-third (one-fifth) of these cores were classified as Class 0 (Class I) YSOs. About two-thirds of the Perseus YSOs are associated with the two major young clusters NGC 1333 and IC 348, and the remaining YSOs are either associated with other much smaller clouds, such as Barnard 5, Barnard 1, L1455 and L1448, or sparsely distributed across the whole Perseus cloud region (e.g. Evans et al. 2009; J $\phi$ rgensen et al. 2007).

A systematic investigation of the evolutionary stages and disk properties of the Perseus YSOs with the optical-to-IR SEDs is still lacking. Moderately deep broadband gri imaging data were recently obtained through PMO's Xuyi Schmidt Telescope Photometric Survey of the Galactic Anti-center (XSTPS-GAC; Liu et al. 2014; Zhang et al. 2013, 2014; Yuan et al. 2015, in preparation). In this paper, we combined the gri data with the IR data from 2MASS, Spitzer, and WISE in order to study the physical properties of the central stellar sources, the evolutionary stages and inner disk properties of the Perseus YSOs. Future spectroscopic data from LAMOST on most YSOs will further detail and enhance the broad-band characterization offered in this paper. Section 2 introduces the data and YSO sample analyzed in this work. The color-magnitude diagrams are presented in Section 3 Section 4 presents the results from SED modeling, such as the central stellar masses, ages, and the evolutionary stages of the YSOs. An investigation of the excess dust emission and disk geometry parameters, such as the disk inner radii and outer disk flaring, and implications on the dominant disk clearing processes are given in Section 5 A brief summary of the main results in this work is given in Section 6

\section{SAMPLE AND DATA}

\subsection{Parent Sample of Perseus YSOs}

The most recent census of Perseus YSOs was done by Hsieh \& Lai (2013, HL13) using the photometric data from Spitzer c2d legacy project (Evans et al. 2009), which carried out a wide-field imaging survey of five nearby low-mass star-forming clouds (Serpens, Persues, Ophiuchus, Lupus, and Chamaeleon) with both IRAC and MIPS instruments onboard Spitzer. Instead of simply relying on a cut on one or two color-color and color-magnitude diagrams to separate YSOs from main-sequence stars and background galaxies, HL13 identified YSOs in a multi-dimensional magnitude space. In particular, HL13 used data from the Spitzer SWIRE survey of the ELAIS N1 extragalactic field (Surace et al. 2004) to acquire a control sample for background galaxies, and this control sample was used to define the regions occupied by galaxies in the multi-dimensional magnitude space. The readers are referred to HL13 for more details about the YSO identification procedure.

In total, HL13 identified 469 Perseus YSOs over $3.86 \mathrm{deg}^{2}$ covered by the c2d survey. Adopting a distance of $250 \mathrm{pc}$ for the Perseus cloud, $3.86 \mathrm{deg}^{2}$ corresponds to about $73.6 \mathrm{pc}^{2}$ (Evans et al. 2009). Among the 469 YSOs, $21 \%$ was classified as Class 0/I sources, $10 \%$ as Class FLAT sources, $58 \%$ as Class II sources, and $10 \%$ as Class III sources based on the 2MASS $K_{s}$ to MIPS $24 \mu \mathrm{m}$ spectral indices $\alpha$. We note that 429 of the 469 YSOs have detections in at least 3 IR bands, and thus the identification of these 429 YSOs in the multi-magnitude space should be more reliable than the rest 40 . In the following, the 429 YSOs will be regarded as the parent sample, and our subsample selection and analysis will be based on these 429 YSOs.

\subsection{Data}

\subsubsection{Broadband gri Photometry from XSTPS-GAC}

From the fall of 2009 to the spring of 2011, the XSTPS-GAC observing project carried out an imaging survey toward the Galactic anti-center in SDSS gri bands with the PMO's Xuyi 1.04/1.20m Schmidt Telescope. The survey covers the sky area from RA $\sim 45^{\circ}$ to $135^{\circ}$ and DEC $\sim-10^{\circ}$ to $60^{\circ}$, plus an extension of $\sim 900 \mathrm{deg}^{2}$ toward the M31/M33 direction. With an exposure time of 90 seconds, the survey reaches $r_{\lim } \sim 19$ in the $r$ band at $10 \sigma$ for point sources. The astrometry (accurate to $\sim$ 
$0.1^{\prime \prime}$ ) was calibrated against the PPMXL catalog (Roeser et al. 2010), and the PSF-fitting photometry was calibrated against the SDSS DR8 catalog using the overlapping sky area with an accuracy of $2 \%$. Given the importance of optical bands in constraining the properties of central stellar sources of YSOs, XSTPS-GAC point sources with $\mathrm{S} / \mathrm{N}>2\left(r_{\lim } \simeq 21 \mathrm{mag}\right)$ will be used in this work.

\subsubsection{Spitzer Data from the c2d Project}

As mentioned above, the Perseus cloud has been observed by the c2d project in the Spitzer IRAC 3.6 (IR1), 4.5 (IR2), 5.8 (IR3), and 7.9 (IR4) $\mu \mathrm{m}$ and MIPS 24 (M1), 70 (M2), and 160 (M3) $\mu \mathrm{m}$ bands. All data, including imagery and point-source photometry (through PSF fitting) catalogs for IRAC, M1 and M2 were processed and released by the c2d team. In this work, we used the HREL (high reliability) source catalog provided by the c2d project 1 .

\subsubsection{MASS and WISE Data}

The $J H K_{s}$ photometry was taken from the 2MASS Point Source Catalog which reaches a $K_{s}$-band limiting magnitude of $14.3 \mathrm{mag}$ at $10 \sigma$. The Wide-field Survey Explorer (WISE) survey mapped the whole sky in four IR broad bands, i.e. 3.4 (W1), 4.6(W2), 12(W3) and 22(W4) $\mu \mathrm{m}$, with a $5 \sigma$ limiting magnitude of 16.6, 15.6, 11.3 and 8.0 mag respectively for the four bands. In this work, we used the ALLWISE Source Catalog 2 which includes enhanced photometric sensitivity and accuracy, and improved astrometric precision compared to the 2012 WISE All-Sky Data Release.

\subsection{Our Working Sample}

In this work, we selected a subsample of 211 Perseus YSOs from the HL13 parent sample. The 211 YSOs were selected by cross-matching the HL13 catalog with all the above data sets, with a requirement that each source should have $J H K_{s}$, IRAC or WISE, M1 or W4, and at least one optical band available. Among the 211 YSOs, 102 have $g$-band detections with S/N > 2, 151 have $r$-band detections, and 198 have $i$-band detections. We point out that 78\% (99\%) of the $g$-band detections have $\mathrm{S} / \mathrm{N}>10$ (5), $85 \%$ (99\%) of the $r$-band detections have $\mathrm{S} / \mathrm{N}>10$ (5), and 94\% (100\%) of the $i$-band detections have $\mathrm{S} / \mathrm{N}$ $>10$ (5). In addition, 27 of our sample YSOs have M2 detections. Optical photometry of the 211 YSOs is given in Table 1

Spatial distribution of the Perseus YSOs is shown in Figure 1, the $110 \mathrm{GHz}{ }^{13} \mathrm{CO}$ integrated intensity map from the The Coordinated Molecular Probe Line Extinction Thermal Emission Survey of Star Forming Regions (COMPLETE, Goodman et al. 2005; Ridge et al. 2006) project is overlaid for comparison. As already known, most the Perseus YSOs are associated with the two major clusters IC 348 and NGC 1333. In particular, 83 of the 211 YSOs are within $15^{\prime}(\sim 1.7 \mathrm{pc}$ at a distance of $320 \mathrm{pc}$, e.g. Belikov et al. 2002; Evans et al. 2009; Strom et al. 1974; de Zeeuw et al. 1999) of IC 348, and 43 are within $15^{\prime}(\sim 1.3 \mathrm{pc}$ at a distance of $250 \mathrm{pc}$, e.g. Evans et al. 2009) of NGC 1333.

Figure2 2 shows histograms of the IR1 mag and the spectral indices $\alpha\left(K_{s}-\mathrm{M} 1\right)$ for the parent sample and our working subsample. As can be seen, our 211 YSOs are expected to be statistically unbiased at least at IR1 $\lesssim 10 \mathrm{mag}$, which would correspond to a stellar mass of $\sim 0.9 M_{\odot}$ at an age of $\sim 3 \mathrm{Myr}$ for a distance modulus $(m-M)_{0}=7.5$ (corresponds to a distance of $320 \mathrm{pc}$ for IC 348), according to the PMS evolutionary tracks of Baraffe et al. (1998). Moreover, since hot dust of the circumstellar disks may contribute significantly to the IR1 emission, our subsample of YSOs may be statistically unbiased down to $M_{\star}$ of slightly below $0.9 M_{\odot}$. In addition, most of our YSOs have $\alpha \lesssim 0.0$, implying that our subsample is dominated by Classes FLAT, II and III YSOs. The spectral index $\alpha\left(K_{s}-\mathrm{M} 1\right)$ (Evans et al. 2009), which quantifies the spectral slope from $K_{s}$ to Spitzer $24 \mu \mathrm{m}$, was obtained from a linear fit to logarithms of all available photometry between $K_{s}$ and M1. Note that for sources without M1 data we used the W4 data instead for determining the spectral indices.

\footnotetext{
1 http://irsa.ipac.caltech.edu/data/SPITZER/C2D/

2 http://wise2.ipac.caltech.edu/docs/release/allwise/
} 


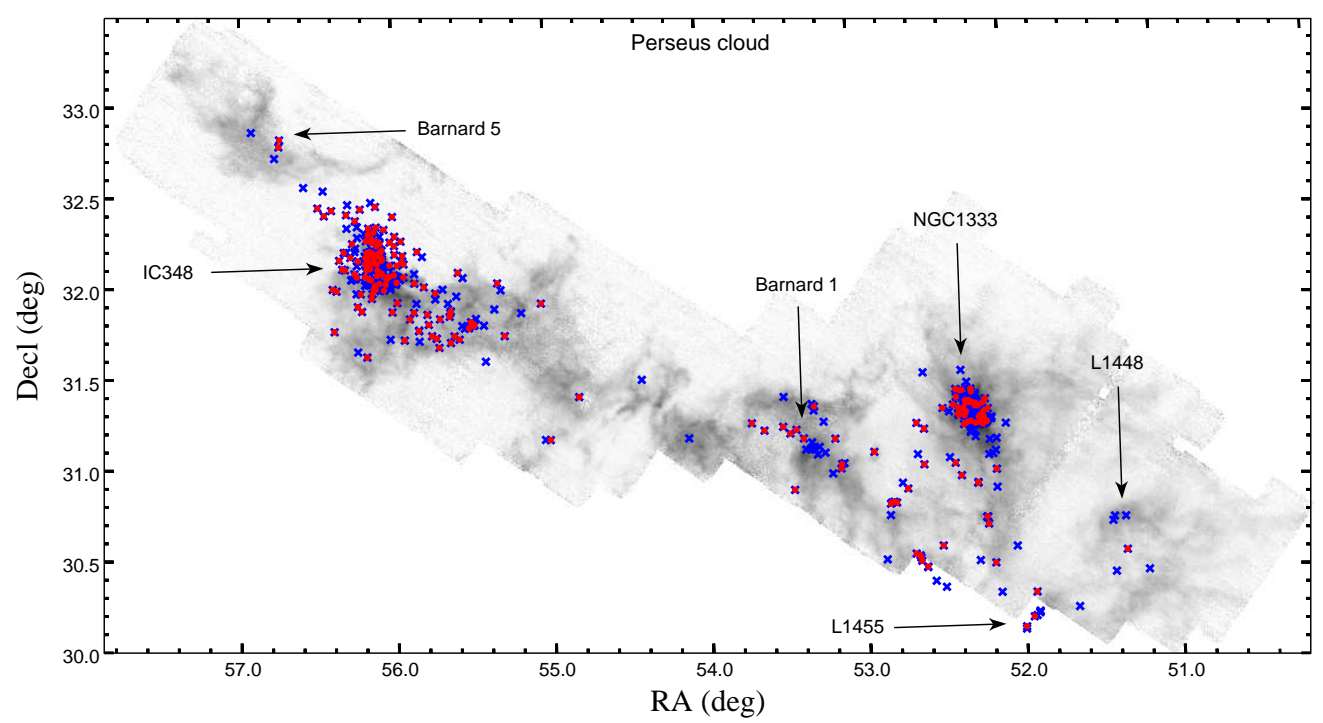

Fig. 1 Spatial distribution of the Perseus YSOs is over plotted on the FCRAO $110 \mathrm{GHz}{ }^{13} \mathrm{CO}$ integrated intensity map (greyscale, FWHM $\simeq 46$ ") from the COMPLETE project. The small red circles mark the 211 YSOs studied in this work, and the blue crosses mark the parent sample of 429 YSOs which have at least 3 band detections in the IR wavelength range from 2MASS $J$ to MIPS $24 \mu \mathrm{m}$. Several well-studied clusters or cores are also annotated in the figure.

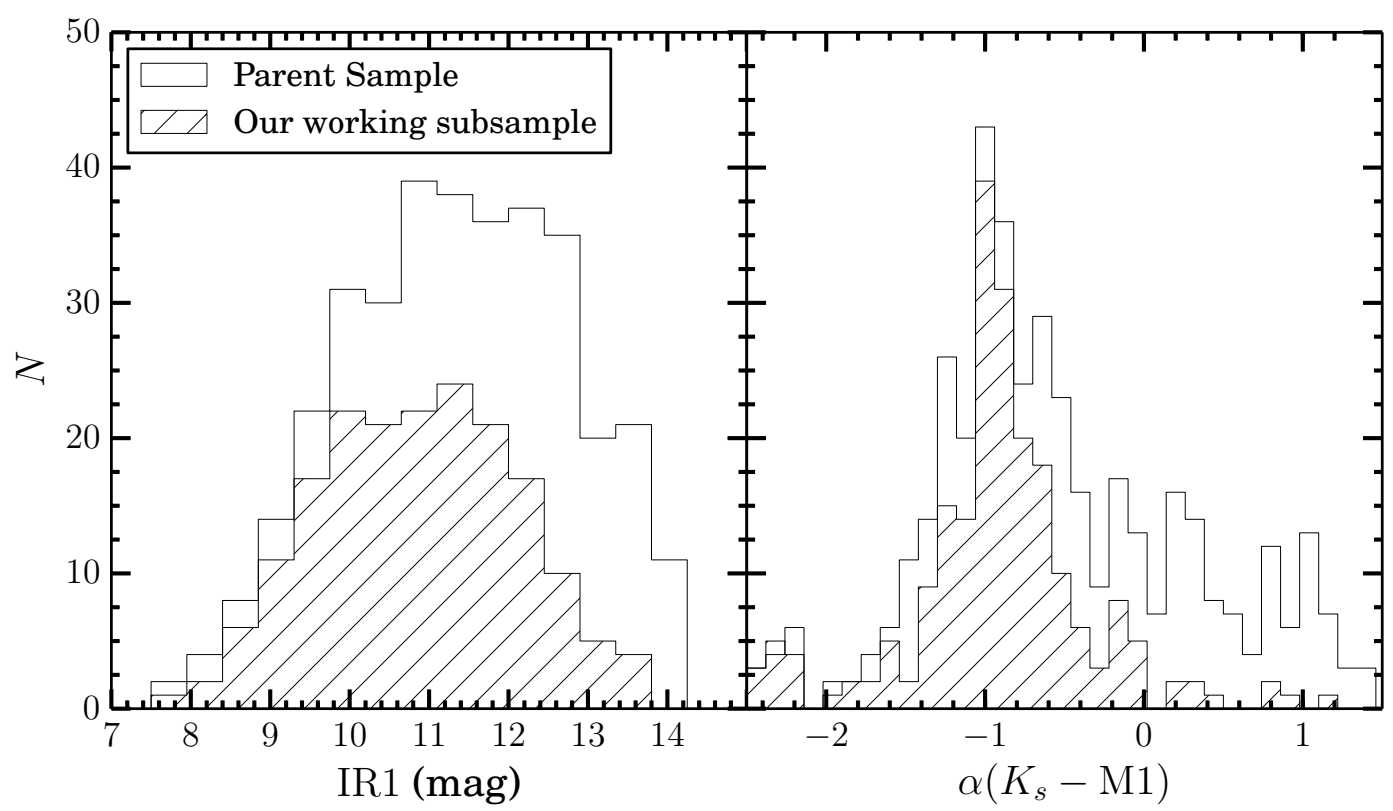

Fig. 2 Histograms of the IRAC 3.6 $\mu \mathrm{m}$ (IR1) magnitude (left) and spectral index $\alpha\left(K_{s}-\mathrm{M} 1\right)$ (right) of wavelength ranges from $K_{s}$ to MIPS $24 \mu \mathrm{m}$ for the parent sample (open) and our working subsample (hatched) of YSOs. 

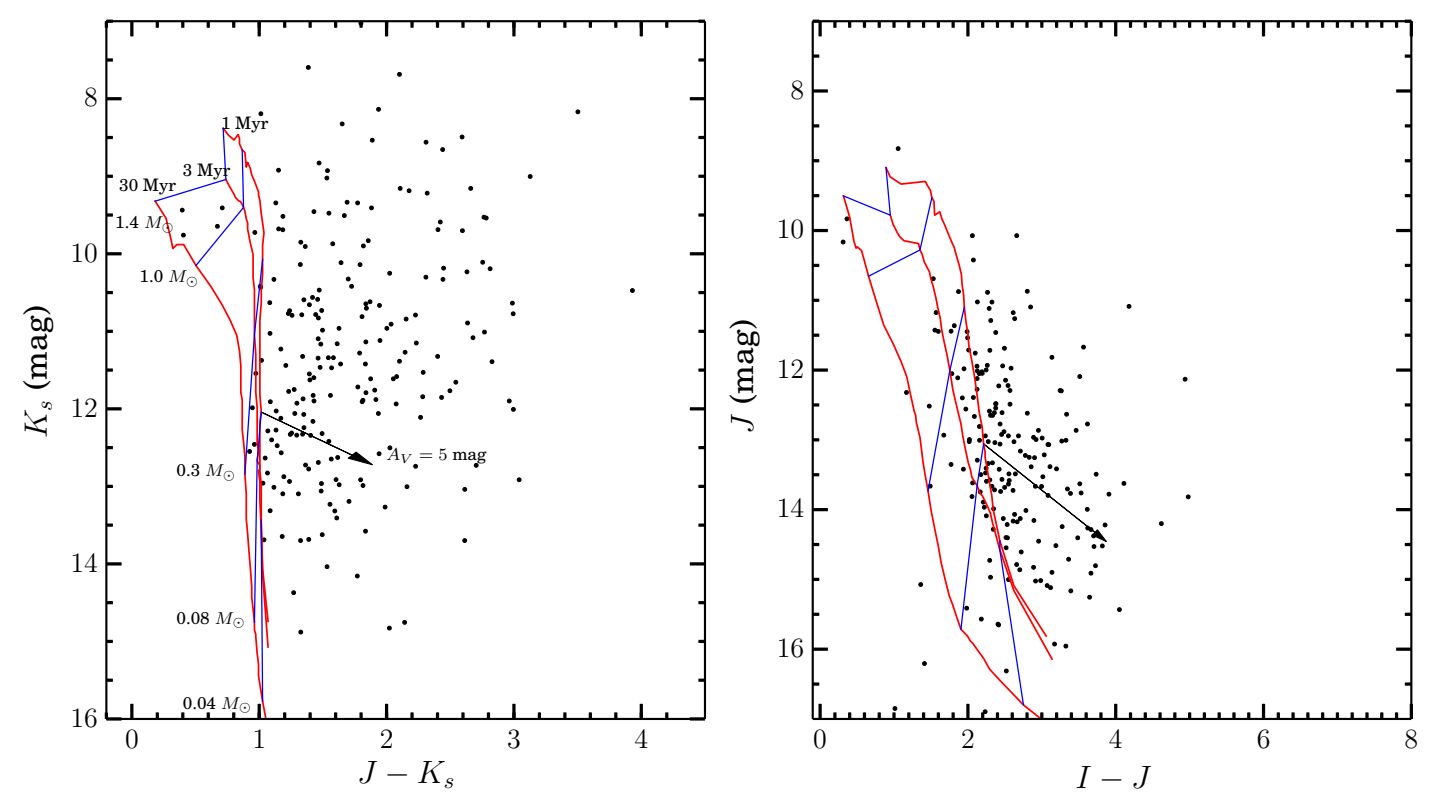

Fig. $3 J-K_{s}$ vs. $K_{s}$ (left) and $I-J$ vs. $J$ (right) color-magnitude diagrams. The left panel shows the distribution for all of the 211 YSOs (filled circles) studied in this work, and the right panel shows the distribution for 198 YSOs with $i$-band detection. Overplotted are stellar evolutionary tracks of Baraffe et al. (1998) for a stellar mass range of $0.02-1.4 M_{\odot}$ at three different ages $(1,3$, and $30 \mathrm{Myr})$. The black arrow in each panel marks the 5-mag visual extinction vector, assuming the Fitzpatrick (1999) extinction law with $R_{V}=3.1$. The evolutionary tracks and extinction vectors shown in the right panel are the same as that in the left panel. A distance modulus $(m-M)_{0}=7.0$ for the Perseus YSOs is adopted.

\section{COLOR-MAGNITUDE DIAGRAMS}

The color-magnitude diagrams for our sample are shown in Figure 3 The evolutionary models for lowmass stars and brown dwarfs from Baraffe, Chabrier, \& Allard (1998) are also plotted in Figure 3 to be compared with our data. When plotting the evolutional models in Figure 3 , the $J H K$ photometry on CIT system as provided by Baraffe et al. (1998) was transformed to the 2MASS photometric system, and our SDSS $i$ magnitude was transformed to the Cousins $I$ magnitude using the transformation equation determined by Lupton (2005). The transformation equation of Lupton (2005) involves Cousins $I$, SDSS $r$, and SDSS $i$. Among the 198 YSOs that have $i$-band detection, 60 do not have $r$-band detection. To put these 60 YSOs on the $I-J$ vs. $J$ diagram (right panel of Figure 3), we adopted a median $I-i=$ -0.82 , as determined from the YSOs with both $r$ and $i$ detections, to transform SDSS $i$ to Cousins $I$.

In Figure 3 most of our YSOs are redder than the pure stellar photosphere emission. A recent study by Chen et al. (2015) found a mean visual interstellar extinction of $\lesssim 1$ mag toward the Perseus region, which is insufficient to explain the red colors of most YSOs, especially for their distribution on the $J-K_{s}$ vs. $K_{s}$ diagram. Therefore, as expected, hot dust emission from the inner circumstellar disks of YSOs contributes significantly to the $K_{s}$ band. The comparison with theoretical evolutionary tracks implies that the masses of our YSOs are mostly above the substellar limit $\left(\sim 0.08 M_{\odot}\right)$. The fact that evolutionary tracks at different masses and ages are well separated on the color-magnitude diagram involving $I$ band data suggests the importance of optical bands in constraining the properties of the central stellar sources of YSOs. 


\section{SED MODELING}

\subsection{The Method}

With the broadband SEDs in hand, we used the online SED fitting tool developed by R06 and Robitaille et al. (2007) to extract the relevant physical properties of YSOs and their circumstellar disks. This online fitting tool offers the possibility of fitting YSO SEDs with a precomputed grid of 200,000 synthetic SEDs computed at 10 viewing angles. The model SEDs account for the contribution from central stellar photosphere emission, circumstellar disks, and infalling envelopes. In particular, the stellar photosphere emission is modeled with two parameters, i.e. stellar luminosity and temperature; The disk is treated as a standard flared accretion disk and the resultant emission is modeled with six parameters, i.e. the disk mass $\left(\sim 0.001-0.1 M_{\odot}\right)$, inner radius, outer radius $(1-10,000 \mathrm{AU})$, accretion rate, scale height factor and flaring angle; The envelope emission is modeled with four parameters, i.e. envelope accretion rate, outer radius, cavity density $\left(10^{-22}-8 \times 10^{-20} \mathrm{~g} \mathrm{~cm}^{-3}\right)$ and cavity opening angle. In addition, the central stellar masses $\left(0.1-50 M_{\odot}\right)$ and ages $(0.001-10 \mathrm{Myr})$ were constrained by comparing the stellar luminosity and temperature with the PMS evolutionary tracks of Bernasconi \& Maeder (1996) and Siess et al. (2000).

Before proceeding to the SED modeling for our data, we point out some limitations of the R06 SED models (Robitaille 2008) that may be relevant to our current work. Firstly, the models do not include the case for multiple central stellar sources, which can affect the size of the disk/envelope inner holes and thus influence the near- to mid-IR emission. Secondly, there exist several sets of different PMS evolutionary tracks in the literature, besides the Siess et al. tracks as adopted by the R06 SED models, other popular PMS tracks include Swenson et al. (1994), D'Antona \& Mazzitelli (1997), Baraffe et al. (1998), Palla \& Stahler (1999), Yi et al. (2003), and Dotter et al. (2008). Adopting different tracks can lead to systematic differences on the fitted stellar parameters (e.g. Fang et al. 2013; Hillenbrand et al. 2008), and the systematic effects are especially significant for sub-solar mass stars at young ages. In particular, uncertainties of age estimation from different tracks for sub-solar mass stars can be up to 0.75 dex at young ages ( $<10 \mathrm{Myr}$, Hillenbrand et al. 2008). Thirdly, the dust opacity law assumed in the models may not be accurate, which would affect the determination of disk/envelope accretion and mass.

When fitting the SEDs, a 5\% absolute flux calibration uncertainty was added in quadrature to the gri uncertainties, a $10 \%$ uncertainty was added to $J H K_{s}$ and IRAC data uncertainties, and a $20 \%$ uncertainty was added to the M2 data uncertainties (Evans et al. 2009). In addition, when both IR1, IR2 and W1, W2 data are available, IR1 and IR2 were used in the fitting due to the higher resolution of IRAC data. An aperture of $10^{\prime \prime}$ was used in the fitting. In addition, the distance to YSOs was allowed to vary from 0.2 to $0.35 \mathrm{kpc}$, and the foreground interstellar extinction $A_{V}$ was allowed to vary from 0.3 to $30 \mathrm{mag}$, with the lower limit of $A_{V}$ being chosen based on the Perseus extinction map as determined by Chen et al. (2014). Besides the best-fitting model parameters, all the subsequent well-fit models

with reduced $\chi_{\mathrm{r}}^{2}-\chi_{\mathrm{r}, \text { best }}^{2}<2$ were used to define the minimum and maximum acceptable physical parameters.

\subsection{The Results}

The range of wavelength coverage determines what physical parameters can be constrained from SED modeling. A thorough investigation about how the wavelength range of data affects the determination of different physical properties of YSOs was given by R06. Given our wavelength coverage from optical to MIPS $24 \mu \mathrm{m}$ (or WISE $22 \mu \mathrm{m}$ ), we expect to roughly constrain the central stellar source luminosity, extinction, and the circumstellar disk luminosity. Although subject to much larger uncertainties than constraints from spectroscopic data, the central stellar masses and ages can still be roughly constrained from broadband SED modeling to statistically investigate a large sample, like the one presented in this work. Moreover, while the masses of the circumstellar disks and envelopes cannot be reliably constrained unless one have far-IR to submm data, SED modeling for wavelength ranges short of far-IR 
can still be used to statistically constrain the evolutionary stages of YSOs. R06 found that at least 3 different evolutionary stages of YSOs can be statistically distinguished based on the fitted stellar masses normalized envelope accretion rates $\left(\dot{M}_{\text {env }} / M_{\star}\right)$ and disk masses $\left(M_{\text {disk }} / M_{\star}\right)$. In particular, the Stage I YSOs have significant infalling envelopes and are defined by having $\dot{M}_{\mathrm{env}} / M_{\star}>10^{-6} \mathrm{yr}^{-1}$; Stage II YSOs have optically thick disks and are defined by having $\dot{M}_{\text {env }} / M_{\star}<10^{-6} \mathrm{yr}^{-1}$ and $M_{\text {disk }} / M_{\star}>$ $10^{-6}$; Stage III YSOs have optically thin disks and are defined by having both $\dot{M}_{\mathrm{env}} / M_{\star}<10^{-6} \mathrm{yr}^{-1}$ and $M_{\text {disk }} / M_{\star}<10^{-6}$. Lastly, the near- to mid-IR SEDs are also sensitive to the disk properties, such as the disk inner radius and disk flaring.

Figure 4 shows the SEDs of the 27 YSOs which have M2-band detections and at the same time at least one optical band available. The black solid curve in each panel of Figure 4 is the best-fit model SED, and the grey solid curves represent all subsequent well-fit models with reduced $\chi_{\mathrm{r}}^{2}-\chi_{\mathrm{r}, \text { best }}^{2}<2$. In addition, SEDs of the best-fit stellar photosphere emission (corrected for both the interstellar and circumstellar extinction) are overplotted as dashed curves. By calculating likelihood estimator $\mathrm{e}^{-\chi_{\mathrm{r}}^{2} / 2}$ for each well-fit model with $\chi_{\mathrm{r}}^{2}-\chi_{\mathrm{r} \text {,best }}^{2}<2$ for a given YSO, we construct the probability density function (PDF) and the correspondent cumulative distribution function (CDF) for parameters such as stellar masses $M_{\star}$, ages and disk inner radius $R_{\text {in }}$. The most probable value for each parameter refers to the median of the corresponding PDF, and the confidence interval is defined as covering the central $95 \%$ of the CDF. In what follows in this section, we will present the results for $M_{\star}$, ages, and the evolutionary stages as identified based on the stellar masses normalized disk masses and envelope accretion rates. Discussion about the disk geometry parameters from SED modeling and fractional dust luminosity $L_{\text {dust }} / L_{\star}$, where $L_{\text {dust }}$ (in units of $L_{\odot}$ ) is equal to integral of the best-fit stellar photosphere subtracted SEDs, will be presented in the next section. SED modeling results for some relevant parameters, such as $M_{\star}$, ages, and $R_{\text {in }}$, are listed in Table 2 .

\subsubsection{Stellar Mass Distribution of the Central Stellar Sources}

Stellar mass histogram of our sample is shown in the left panel of Figure 5. As pointed out above, our sample is expected to be statistically unbiased at $M_{\star} \gtrsim 0.9 M_{\odot}$. We overplot the Salpeter stellar initial mass function (IMF; Salpeter 1955) which was scaled to have the same number of stars at $M_{\star}>0.9 M_{\odot}$ with our YSO sample. The error bars in the histogram represent the Poisson noise from number counts. It can be seen that the mass distribution of our YSOs at $M_{\star} \gtrsim 1 M_{\odot}$ is consistent with the Salpeter IMF within the uncertainties. Note that an extended star formation history for the Perseus region might make it not straightforward to compare the accumulated present-day mass function with the simple Salpeter IMF. Although our sample may be subjected to significant incompleteness bias below $1 M_{\odot}$, we note that a flat and broad mass distribution from sub-solar to sub-stellar mass limit, as found in our sample, is in general agreement with previous studies of low-mass clusters such as IC 348 (e.g. Luhman et al. 2003a; Muench et al. 2003), NGC 1333 (e.g. Wilking et al. 2004; Greissl et al. 2007), Trapezium (e.g. Muench et al. 2002) and other nearby clusters (e.g. Andersen et al. 2008; Hillenbrand \& Carpenter 2000; Luhman et al. 2000; Lucas et al. 2005; Luhman 2007; Levine et al. 2006; Moraux et al. 2003; Slesnick et al. 2004; Scholz et al. 2009; Weights et al. 2009). The median $M_{\star}$ of our YSOs is $\simeq 0.3 M_{\odot}$.

Stellar mass distributions of YSOs within $15^{\prime}$ radius of each of the two major clusters IC 348 and NGC 1333 are shown in the left panel of Figure 6. The median stellar masses of YSOs in IC 348 and NGC 1333 are $\simeq 0.3 M_{\odot}$.

\subsubsection{Age Distribution of the Central Stellar Sources}

The age histogram for the whole sample is shown in the right panel of Figure 5, and the age histograms for each of the two major clusters (again defined with a 15' radius) are shown in the right panel of Figure 6 The median stellar age of the whole sample is $\simeq 3.1 \mathrm{Myr}$, and the median age for YSOs in IC 348 and NGC 1333 is $\simeq 2.8$ and 2.5 Myr respectively. A relatively younger age of NGC 1333 than IC 348 is 

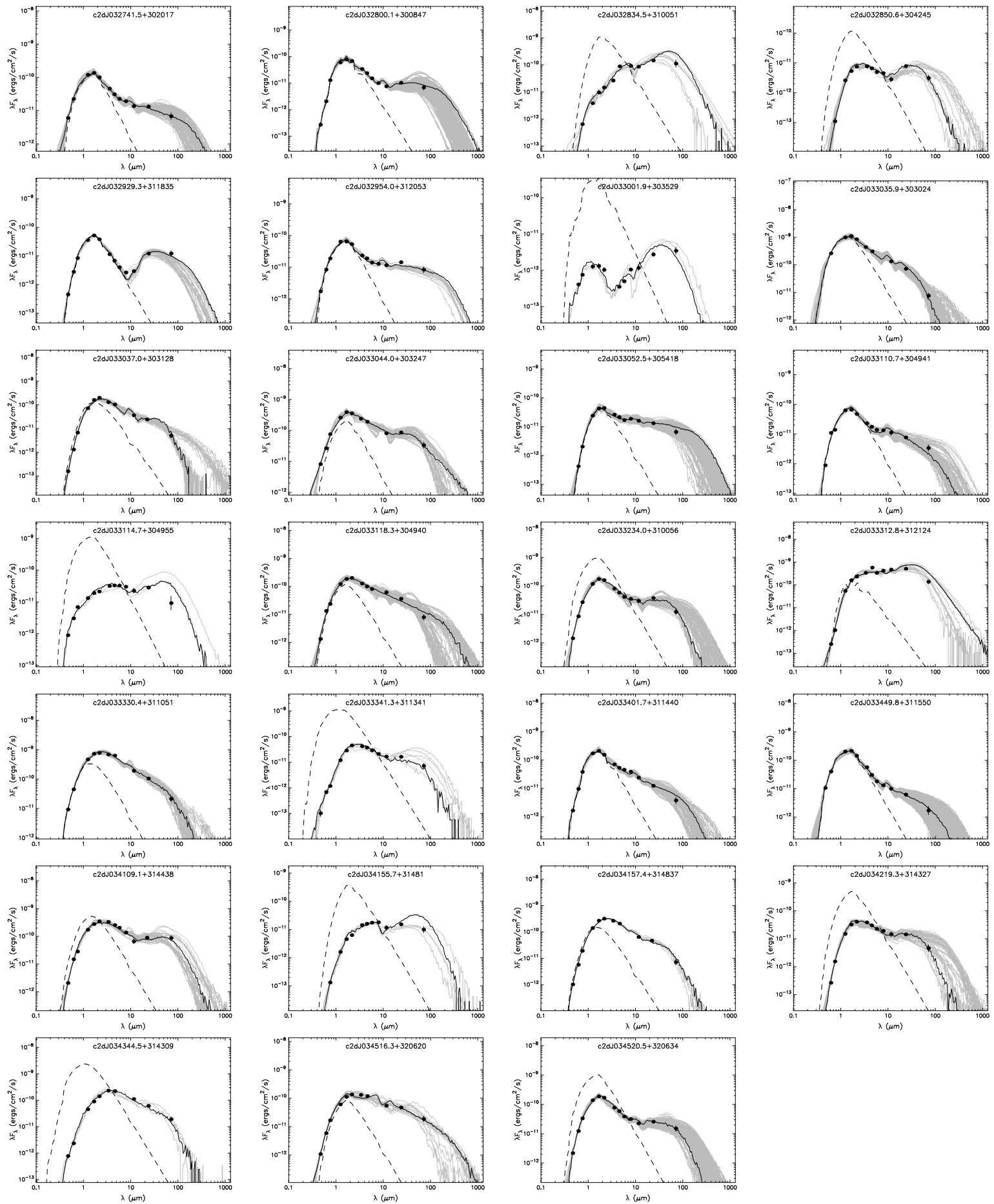

Fig. 4 SEDs of 27 Perseus YSOs. Among our whole sample, these YSOs have at least one optical band, $J H K_{s}$, IRAC or WISE, MIPS $24 \mu \mathrm{m}$ or WISE $22 \mu \mathrm{m}$, and MIPS $70 \mu \mathrm{m}$ available (black points). The black solid curve in each panel represents the best-fit model SED of Robitaille et al. (2007), and the grey curves represent all subsequent well-fit models with $\chi_{\mathrm{r}}^{2}-\chi_{\mathrm{r}, \mathrm{best}}^{2}<2$. The dashed lines illustrate the SEDs of stellar photosphere in the best-fit model, as it would appear to be in the absence of circumstellar dust. 

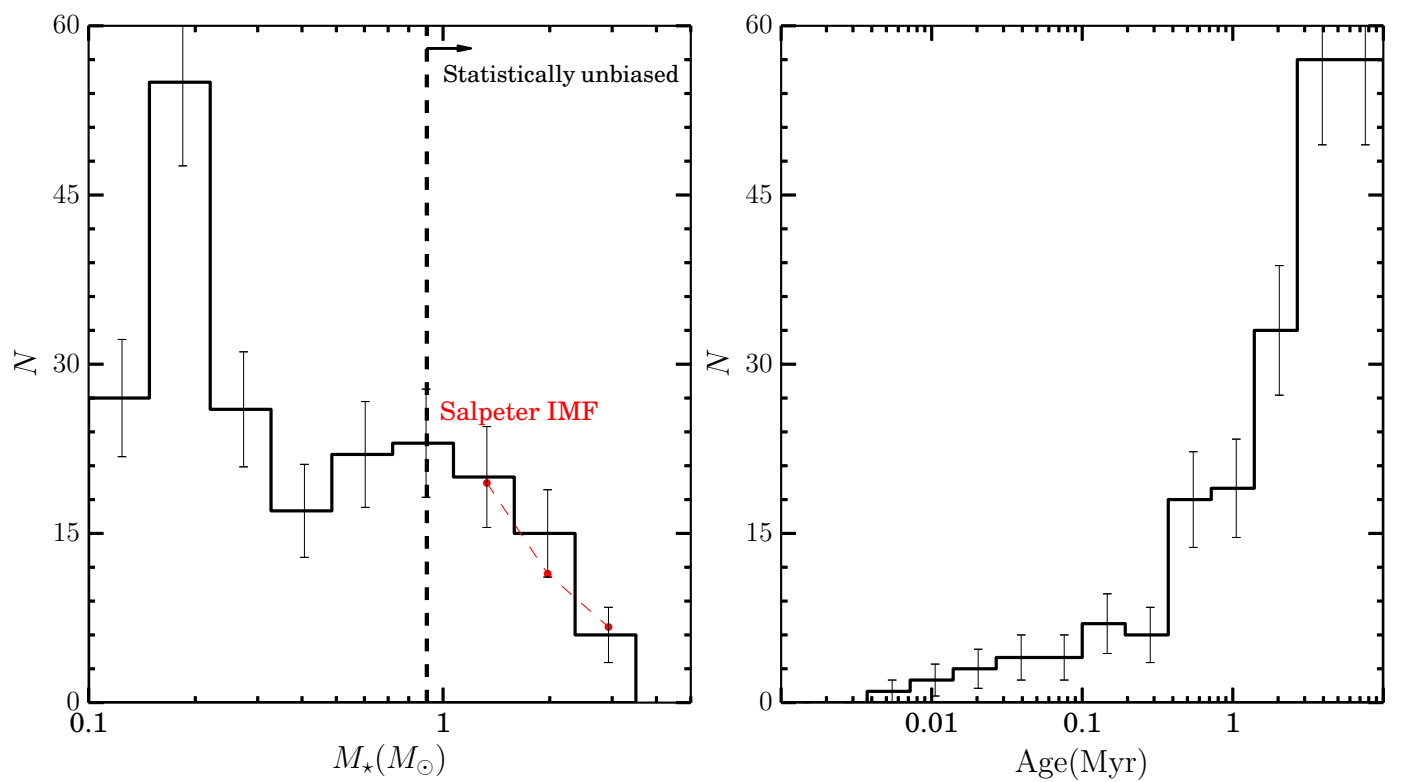

Fig. 5 Histograms of the stellar masses (le ft) and ages (right) for the whole sample of YSOs. The filled red circles in the left panel represents the Salpeter IMF that is scaled to have the same number of observed stars more massive than $0.9 M_{\odot}$.
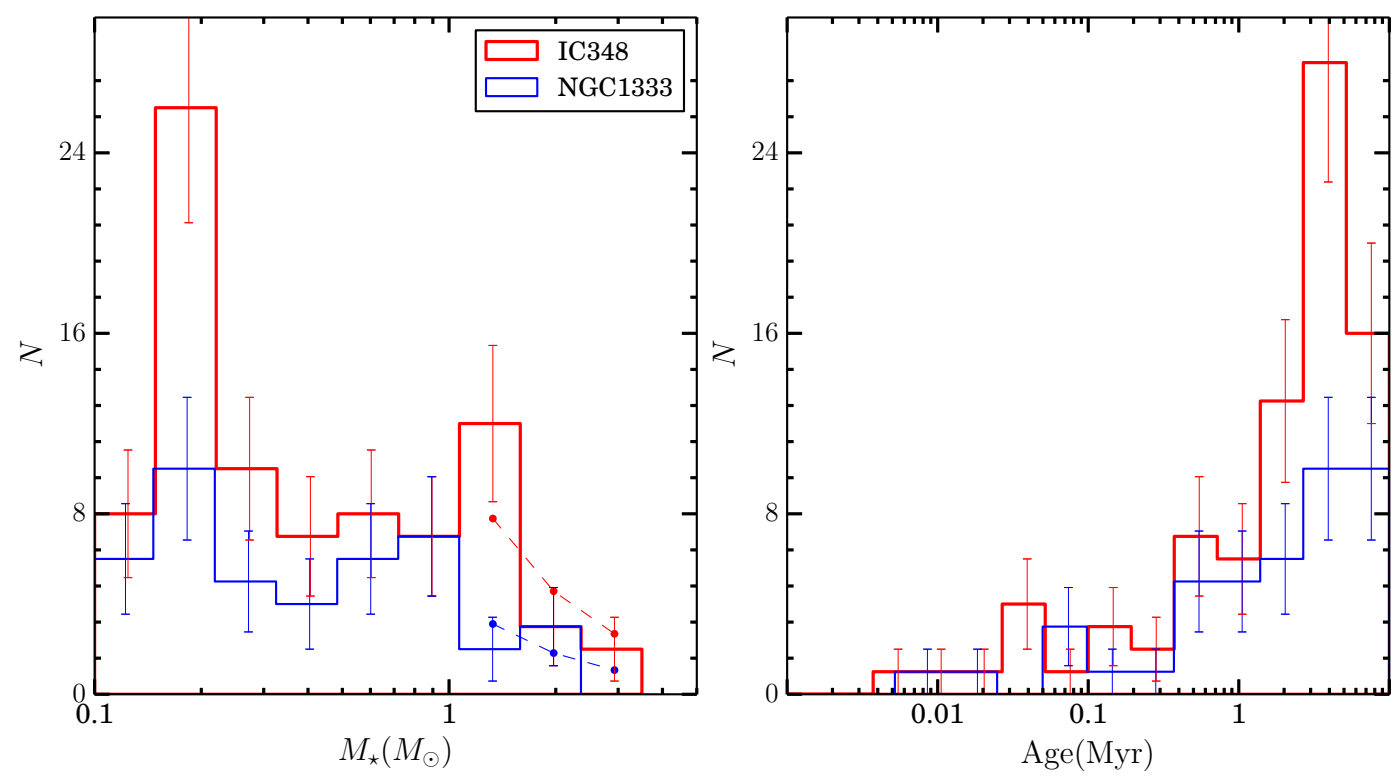

Fig. 6 Histograms of the stellar masses (left) and ages (right) for the two major clusters IC 348 (thick red) and NGC 1333 (thin blue). YSOs within $15^{\prime}$ radius of each of the two clusters are regarded to be associated with the cluster. The filled red (blue) circles in the left panel represent the Salpeter IMF for IC 348 (NGC 1333) that is scaled to have the same number of observed stars more massive than $0.9 M_{\odot}$. 


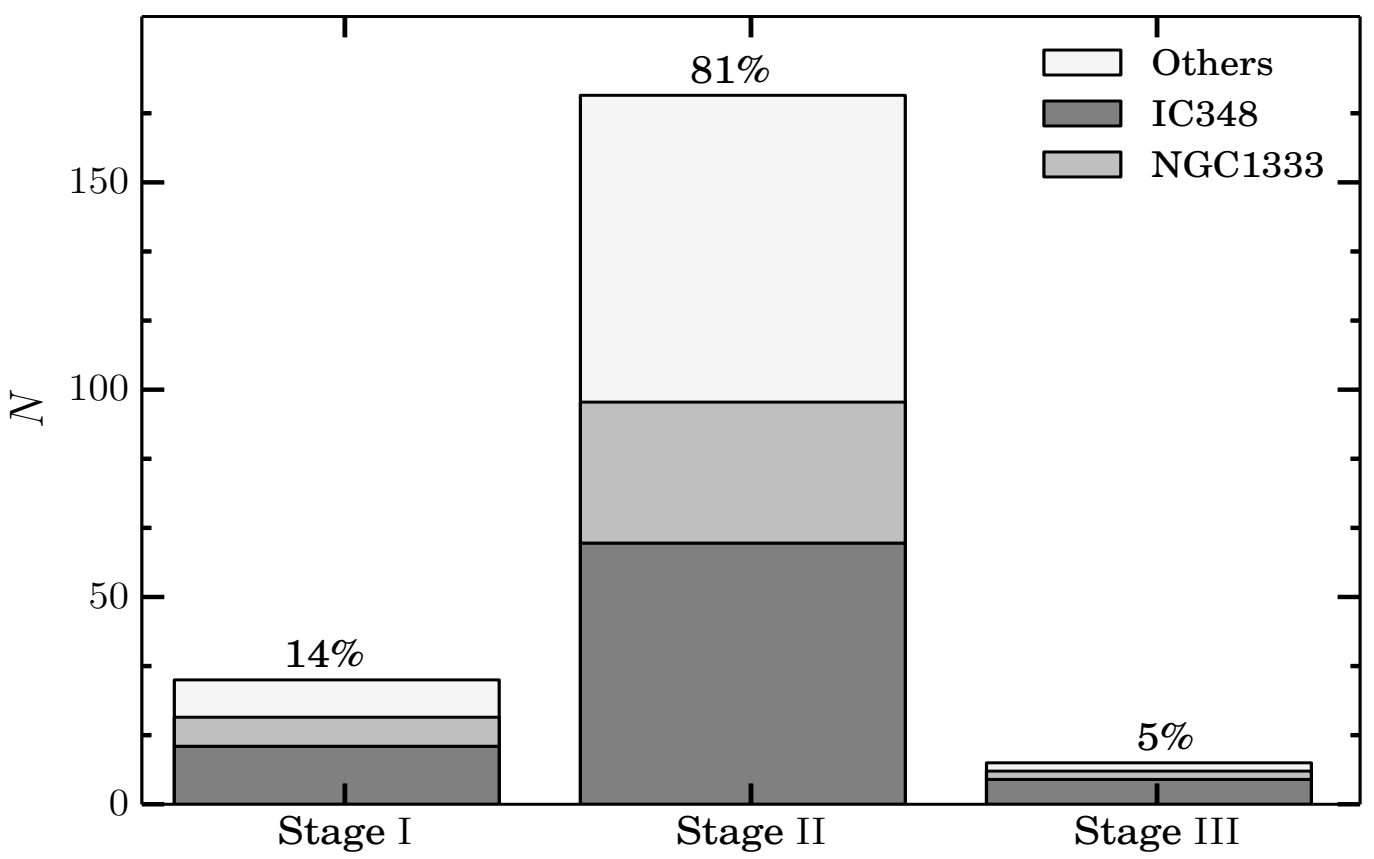

Fig. 7 Classification of evolutionary stages of our sample based on the fractional envelope accretion rates and disk masses.

in line with previous studies, and our age estimate is consistent with previous studies of YSOs in these two clusters (e.g. Herbig 1998; Luhman et al. 2003b; Lada et al. 2006; Winston et al. 2009).

\subsubsection{Uncertainties on Stellar Parameters from SED Modeling}

Determination of the masses and ages of central stellar sources relies on a reasonably accurate constraint on the effective temperature $T_{\text {eff }}$. While it is reasonable to statistically explore the distribution of masses and ages determined from broadband SED modeling for a large sample, results for individual sources may be subject to large uncertainties. In principle, $T_{\text {eff }}$ can be accurately constrained by photospheric absorption lines from optical or near-IR spectroscopy. By comparing our SED-based and the spectroscopy-based $T_{\text {eff }}$ for 75 IC 348 YSOs that have spectroscopic observations in the literature (e.g. Luhman et al. 2003b; Lada et al. 2006; Muzerolle et al. 2006; Muench et al. 2007), we found a median and standard deviation of $T_{\text {eff,SED }}-T_{\text {eff,Spec }}$ of $71(\sim 2 \%)$ and $257 \mathrm{~K}(\sim 7 \%)$ respectively for the 35 objects with $A_{V}<4$ mag and $T_{\text {eff,Spec }}<5000 \mathrm{~K}$, and a median and standard deviation of $T_{\text {eff,SED }}-T_{\text {eff,Spec }}$ of $68(\sim 2 \%)$ and $434 \mathrm{~K}(\sim 12 \%)$ respectively for the 31 objects with $A_{V}>4$ mag and $T_{\text {eff,Spec }}<5000 \mathrm{~K}$. In addition, the remaining 9 objects with $T_{\text {eff,Spec }}>5000 \mathrm{~K}$ have a median and standard deviation of $T_{\text {eff,SED }}-T_{\text {eff, Spec }}$ of $-1333(\sim 24 \%)$ and $1229 \mathrm{~K}(\sim 16 \%)$ respectively. According to the theoretical evolutionary tracks of Baraffe et al. (1998), for a PMS star with $T_{\text {eff }}$ of $3336 \mathrm{~K}$ and an age of $3 \mathrm{Myr}$, which corresponds to a stellar mass of $0.3 \mathrm{M}_{\odot}$, an overestimation of $T_{\text {eff }}$ by $\sim 350 \mathrm{~K}(\sim 2-3$ subclasses in spectral type $)$ at a given luminosity can result in an overestimation of age and mass by factors of 3 and 2 respectively.

\subsubsection{Evolutionary Stages of YSOs}

Similar to Povich et al. (2013), for every YSO, we calculated the accumulated probability $\left(P_{\text {stage }} \propto \sum_{\text {model i }} \mathrm{e}^{-\chi_{\mathrm{i}, \mathrm{r}}^{2} / 2}\right)$ of it being in each of the three Stages (i.e. $\left.P_{\text {StageI }}, P_{\text {StageII }}, P_{\text {StageIII }}\right)$ based 


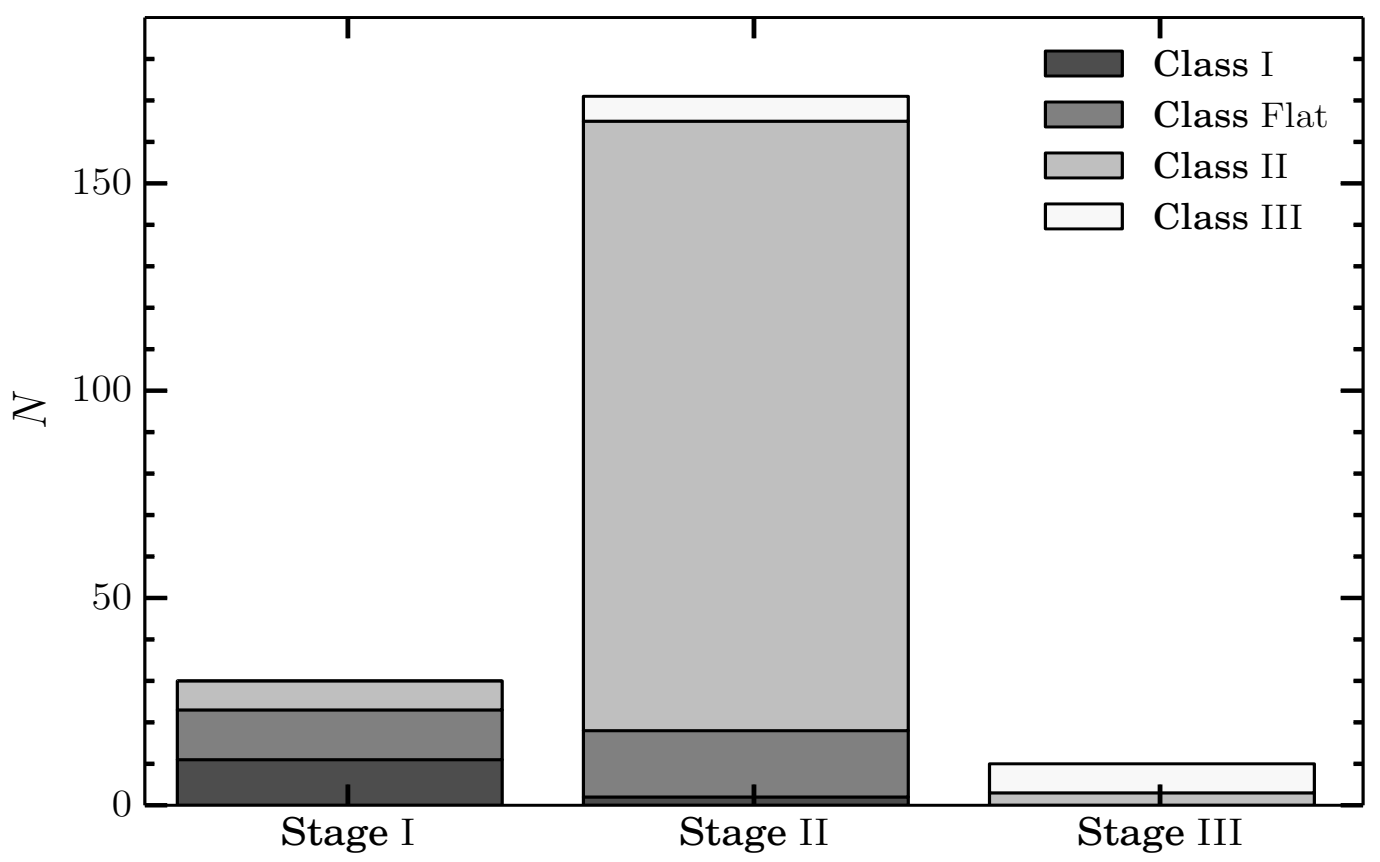

Fig. 8 Breakdown of different evolutionary stages into different $\alpha\left(K_{s}-\mathrm{M} 1\right)$-based Classes.

on all the well-fit models with $\chi_{\mathrm{r}}^{2}-\chi_{\mathrm{r}, \text { best }}^{2}<2$. A YSO is uniquely classified as a Stage I, II, or III object if the normalized $P_{\text {Stage }}>0.67$. The result of our classification is presented in Figure 7 , There are $5 \%$ YSOs that can not be classified as either Stage I, II, or III if adopting the 0.67 probability threshold. These 5\% objects were classified as evolutionary stages that have the highest accumulated probability. The classification of some YSOs into Stage I phase may be subject to relatively large uncertainties. This is because the wavelength coverage of our SEDs is mostly limited to $\lesssim 24 \mu \mathrm{m}$, shortward of which the contribution of excess emission from disks dominates over that from the cool infalling envelopes. Moreover, we note that some of our Stage I YSOs with low IR excess luminosities may be genuine Stage II YSOs with edge-on optically thick disks.

As can be seen from Figure 7 our sample is dominated by Stage II YSOs. Moreover, the fractions of YSOs in different stages are similar for IC 348, NGC 1333, and the other regions. As mentioned in the Introduction section, YSOs have been historically grouped into three or four classes based on the spectral index $\alpha$ determined over the wavelength range from $\sim 2$ to $20 \mu \mathrm{m}$. YSOs of different classes are thought to be in different evolutionary stages (see above for references). R06 showed that there is a general correspondence between the modeling-based "Stages" and $\alpha$-based "Classes", in the sense that Stage I is expected to include the Class 0/I, Stage II is analogous to Class II, and Stage III to Class III. However, as a set of purely empirical criteria, the Class scheme can be sometimes misleading.

Figure 8 presents the breakdown of each Stage into different Classes. As is shown, a vast majority (94\%) of Class II objects are grouped into the Stage II, and the majority (85\%) of Class I objects are grouped into the Stage I. It is noteworthy that the dominant physical Stages for Class FLAT YSOs are uncertain, with about $43 \%$ being in Stage I and the remaining 57\% in Stage II. Likewise, the dominant physical Stages for Class III YSOs are also uncertain, with about $46 \%$ of them being in Stage II and the remaining 54\% in Stage III. 


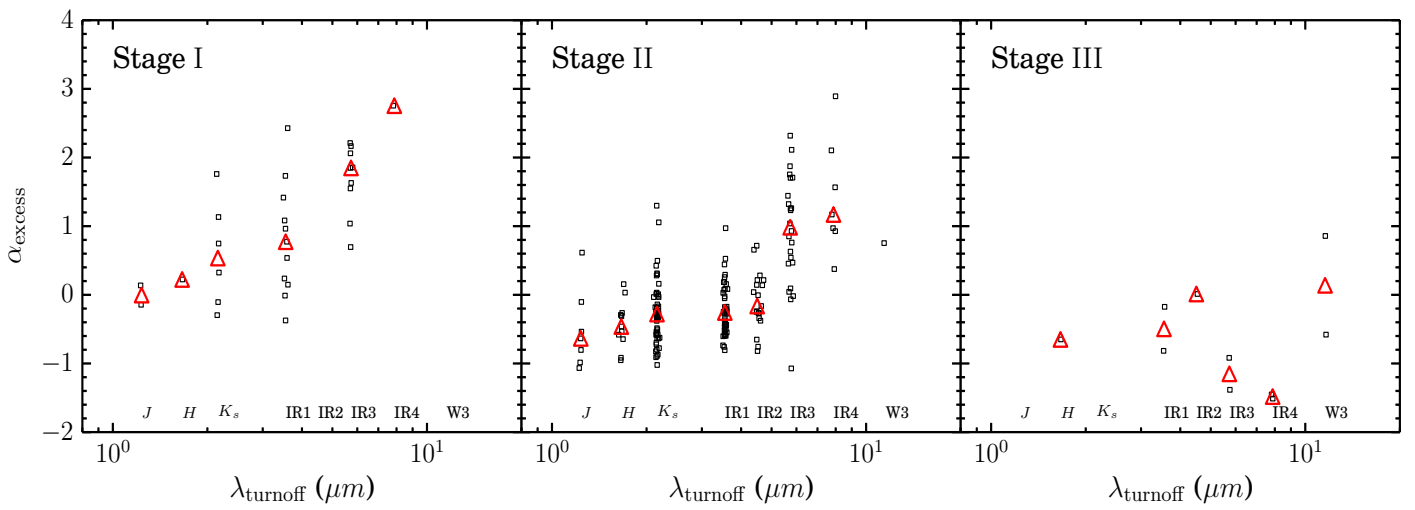

Fig. 9 Distribution of $\alpha_{\text {excess }}$ vs. the wavelength bands $\lambda_{\text {turnoff }}$ longward of which IR excesses are observed. Stages I, II and III YSOs are shown separately in the left, middle and right panels. Median of $\alpha_{\text {excess }}$ at each individual $\lambda_{\text {turnoff }}$ of different Stages was shown as red triangles. Note that data points at a given wavelength band were slightly shifted randomly in the horizontal direction for clarity. See the text for details.

\section{PROPERTIES OF THE CIRCUMSTELLAR DISKS}

The near- to mid-IR excesses above the stellar photosphere level probe the disk properties, such as the disk luminosity (basically an integral of the IR excesses) and disk geometry (e.g. Dullemond et al. 2007; Espaillat et al. 2013; Hughes et al. 2010; Kim et al. 2009; Merín et al. 2010). In particular, disk flaring (e.g. Kenyon \& Hartmann 1987) and radius of the inner disk edge are the two primary disk geometry parameters that shape the SED of IR excesses. As the disk evolves, dust grains in the inner circumstellar disks may gradually settle down (e.g. Dullemond \& Dominik 2005) or be cleared out dynamically (Lubow \& D'Angelo 2006) or through photoevaporation (Alexander et al. 2006a), which leads to a suppression of emission excesses progressively from near- to mid-IR wavelengths. Features of the IR SEDs that are closely related to the disk clearing and flaring include the longest measured wavelength $\lambda_{\text {turnoff }}$ shortward of which the emission is consistent with being purely from stellar photosphere, and the spectral index $\alpha_{\text {excess }}$ at $\lambda>\lambda_{\text {turnoff }}$ (e.g. Cieza et al. 2007; Harvey et al. 2007; Merín et al. 2008).

\section{$5.1 \alpha_{\text {excess }}$ vs. $\lambda_{\text {turnoff }}$}

$\lambda_{\text {turnoff }}$ is closely related to the physical scales of the inward disk truncation or clearing radius (e.g. Calvet et al. 2002; Rice et al. 2003), and $\alpha_{\text {excess }}$ is related to both the inward disk clearing and disk flaring which in turn affect the disk temperature gradients. In particular, for an optically thick disk, a larger spectral index corresponds to a shallower temperature gradient (e.g. Beckwith et al. 1990). By comparing the observed SED of each YSO with the best-fit emergent stellar fluxes (be corrected for interstellar extinction), we determined the turnoff wavelength band $\lambda_{\text {turnoff }}$, longward of which $\geq 3 \sigma$ excesses above the stellar photosphere level were observed, and calculated $\alpha_{\text {excess }}$ for wavelength ranges

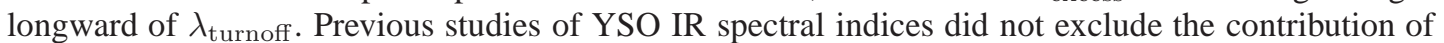
direct stellar photosphere emission. In this work, we focus on $\alpha_{\text {excess }}$ determined for the photospheresubtracted IR SEDs in order to investigate the disk properties. In Table 2 we list spectral indices determined for both the photosphere-included SEDs $\left(\alpha_{\text {turnoff }}\right)$ and photosphere-subtracted SEDs $\left(\alpha_{\text {excess }}\right)$ at $\lambda \geq \lambda_{\text {turnoff }}$. The distribution on $\lambda_{\text {turnoff }}$ vs. $\alpha_{\text {excess }}$ diagram for the subsamples of Stages I, II and III YSOs are shown separately in Figure 9

The median $\alpha_{\text {excess }}$ at each $\lambda_{\text {turnoff }}$ is also indicated as red triangles in Figure 9 . The majority of Stage I YSOs have $\alpha_{\text {excess }} \gtrsim 0.0$, whereas the majority of Stage III YSOs have $\alpha_{\text {excess }} \lesssim 0.0$. Compared to the Stages I and III YSOs, the Stage II YSOs have a larger range of $\alpha_{\text {excess }}$ from $\sim-1$ to 3 . The 


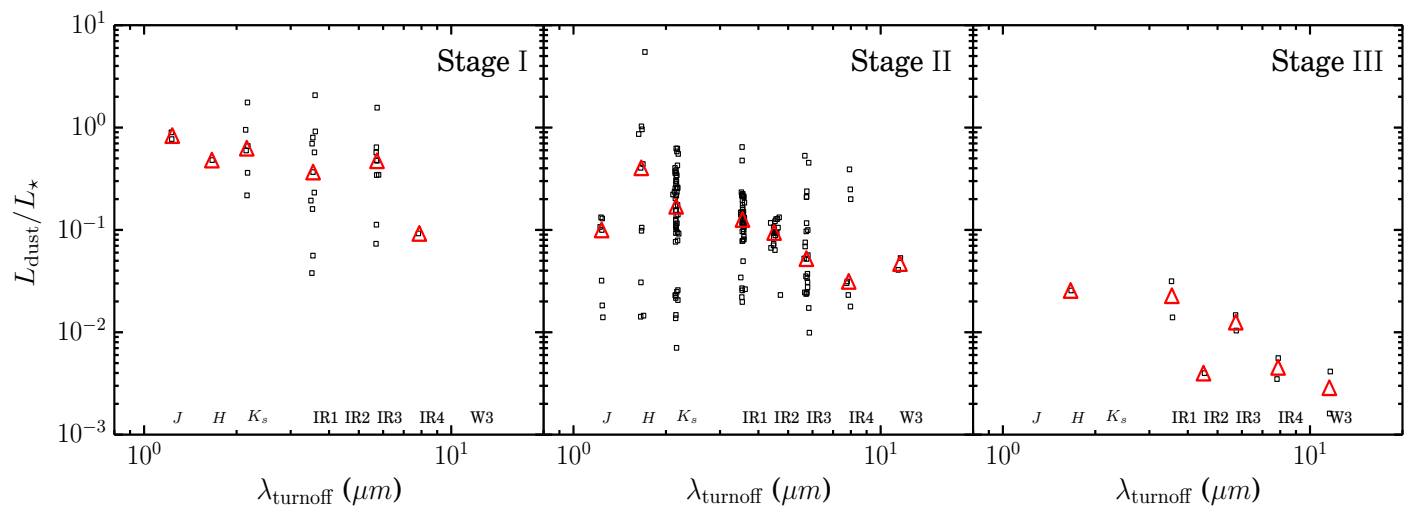

Fig. 10 Distributions of the fractional dust luminosity $L_{\text {dust }} / L_{\star}$ vs. $\lambda_{\text {turnoff }}$ YSOs of different evolutionary stages are plotted separately in different panels. The median $L_{\mathrm{dust}} / L_{\star}$ at different $\lambda_{\text {turnoff }}$ is represented as red triangles.

median $\alpha_{\text {excess }}$ gradually increases with increasing $\lambda_{\text {turnoff }}$ for both Stages I and II YSOs. No obvious trend of the median $\alpha_{\text {excess }}$ with $\lambda_{\text {turnoff }}$ is found for the Stage III YSOs. In addition, there is a hint that the standard deviation of $\alpha_{\text {excess }}$ increases with increasing $\lambda_{\text {turnoff }}$ for the Stage II YSOs which have the largest sample size. In particular, the standard deviations of $\alpha_{\text {excess }}$ for the Stage II YSOs of different turnoff wavelengths at $\lambda_{\text {turnoff }} \leq$ IR2 and $\geq$ IR3 are $\sim 0.4$ and 0.8 respectively. A smaller spread of $\alpha_{\text {turnoff }}$ at shorter $\lambda_{\text {turnoff }}$ has been observed before (e.g. Cieza et al. 2007, Merín et al. 2008). Cieza et al. (2007) found that all the known Classical T Tauri stars (CTTs), which are defined by having relatively strong nebular emission lines and thus being actively accreting, cluster around $\alpha_{\text {turnoff }} \sim-1.0$ and $\lambda_{\text {turnoff }} \lesssim K_{s}$, whereas the Weak-line T Tauri stars (WTTs) exhibit a much larger spread of $\alpha_{\text {turnoff }}$ and $\lambda_{\text {turnoff }}$.

\subsection{Fractional Dust Luminosity vs. $\lambda_{\text {turnoff }}$}

The ratio of the circumstellar dust luminosity $L_{\text {dust }}$ to stellar luminosity $L_{\star}$, which is also known as the fractional dust luminosity, was found to be correlated with the disk accretion activity (e.g. Kenyon \& Hartmann 1995; Muzerolle et al. 2003). In particular, for mildly flared dusty disks, $L_{\mathrm{dust}} / L_{\star} \gtrsim 0.1-$ 0.2 cannot be simply explained by dust reprocessing of stellar radiation alone (Kenyon \& Hartmann 1995) but indicates that a significant amount of IR excesses may be contributed by self-radiation of an actively accreting disk, whereas YSOs with $0.001 \lesssim L_{\text {dust }} / L_{\star} \lesssim 0.1$ are expected to be mostly evolved objects with weaker or no observable accretion activity (e.g. Cieza et al. 2007). Moreover, most gaspoor debris disks (systems which are dominated by second-generation dust produced by the collision of planetesimals) were found to have $L_{\text {dust }} / L_{\star}$ well below 0.001 (e.g. Currie \& Kenyon 2009; Eiroa et al. 2013; Matthews et al. 2014; Su et al. 2006; Trilling et al. 2008).

We determined $L_{\text {dust }}$ as an integral of the R06 model SED of the circumstellar dust (disk+envelope) that best fits the emergent IR excess emission, and $L_{\star}$ as $\left(R_{\star} / R_{\odot}\right)^{2}\left(T_{\star} / T_{\odot}\right)^{4}$, where $R_{\star}$ and $T_{\star}$ are the stellar radius and effective temperature. The distribution of YSOs of different Stages on the $L_{\text {dust }} / L_{\star}$ vs. $\lambda_{\text {turnoff }}$ diagram is shown in Figure 10, The Stages I and III YSOs are well separated at $L_{\text {dust }} / L_{\star} \sim$ 0.1 , whereas the Stage II YSOs have a range of $L_{\mathrm{dust}} / L_{\star}$ from $\sim 0.01$ to 1 . Moreover, there is a general trend that the median $L_{\text {dust }} / L_{\star}$ decreases with increasing $\lambda_{\text {turnoff }}$ for YSOs of different evolutionary stages, pointing to an inside-out disk clearing of at least the small dust grains. 


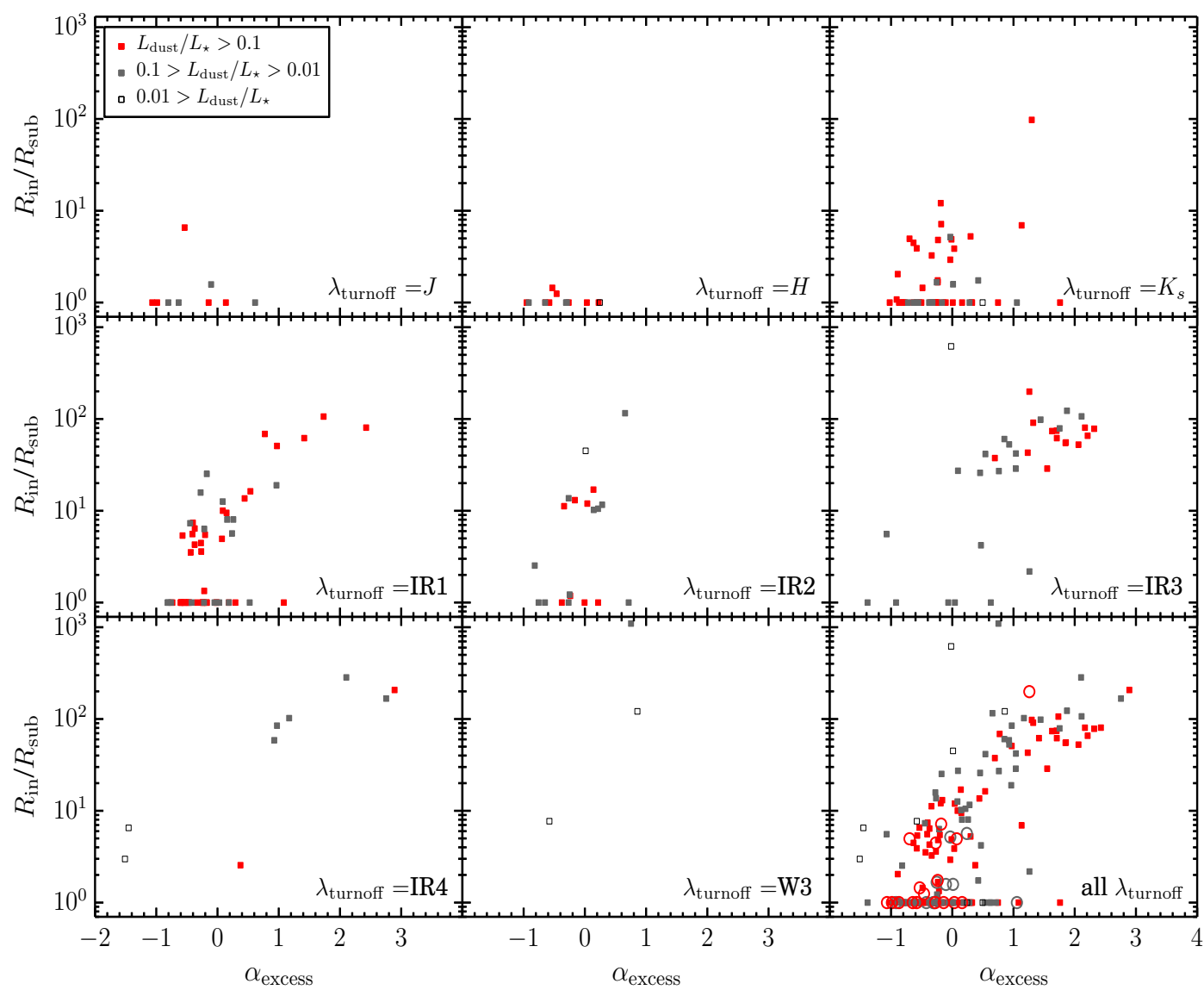

Fig. 11 Distribution of disk inner radius $R_{\text {in }}$ vs. $\alpha_{\text {excess }}$ for different $\lambda_{\text {turnoff }} . R_{\text {in }}$ is normalized to the dust sublimation radius $R_{\text {sub }}$. YSOs with different ranges of $L_{\text {dust }} / L_{\star}$ are plotted with different symbols, as indicated in the top left panel. Distribution for the full sample is shown in the bottom right panel, where objects with $70 \mu \mathrm{m}$ detections are shown as red open circles.

\subsection{Disk Inner Radius vs. $\alpha_{\text {excess }}$}

The inner radius of the dusty disk determines the highest temperature of dust grains orbiting around the central stellar source (e.g. Backman \& Paresce 1993), and thus can affect $\alpha_{\text {excess }}$. Figure 11 shows the relation between $\alpha_{\text {excess }}$ and $R_{\text {in }} / R_{\text {sub }}$ with different $\lambda_{\text {turnoff }}$, where $R_{\text {in }}$ is the disk inner radius, and $R_{\text {sub }}$ is the dust sublimation radius by assuming a sublimation temperature of $1600 \mathrm{~K}$ (R06). The bottom right panel of Figure 11 shows the corresponding distribution for the full sample.

There is a positive correlation between $R_{\text {in }} / R_{\text {sub }}$ and $\alpha_{\text {excess }}$ at $R_{\text {in }} / R_{\text {sub }} \gtrsim 10$ and $\alpha_{\text {excess }} \gtrsim 0.0$,

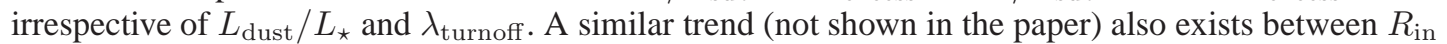
and $\alpha_{\text {excess }}$ at $R_{\text {in }}>0.5 \mathrm{AU}$ and $\alpha_{\text {excess }} \gtrsim 0.0$. We note that a positive correlation was also found between disk inner radii (or hole radii) and disk masses for 35 c2d YSOs by Merín et al. (2010).

\subsection{Disk Flaring vs. $\alpha_{\text {excess }}$}

Compared to a completely flat disk geometry, a flaring geometry increases the disk area that confronts the stellar radiation at large radii, and thus enhances the mid- to far-IR emission (e.g. Kenyon 


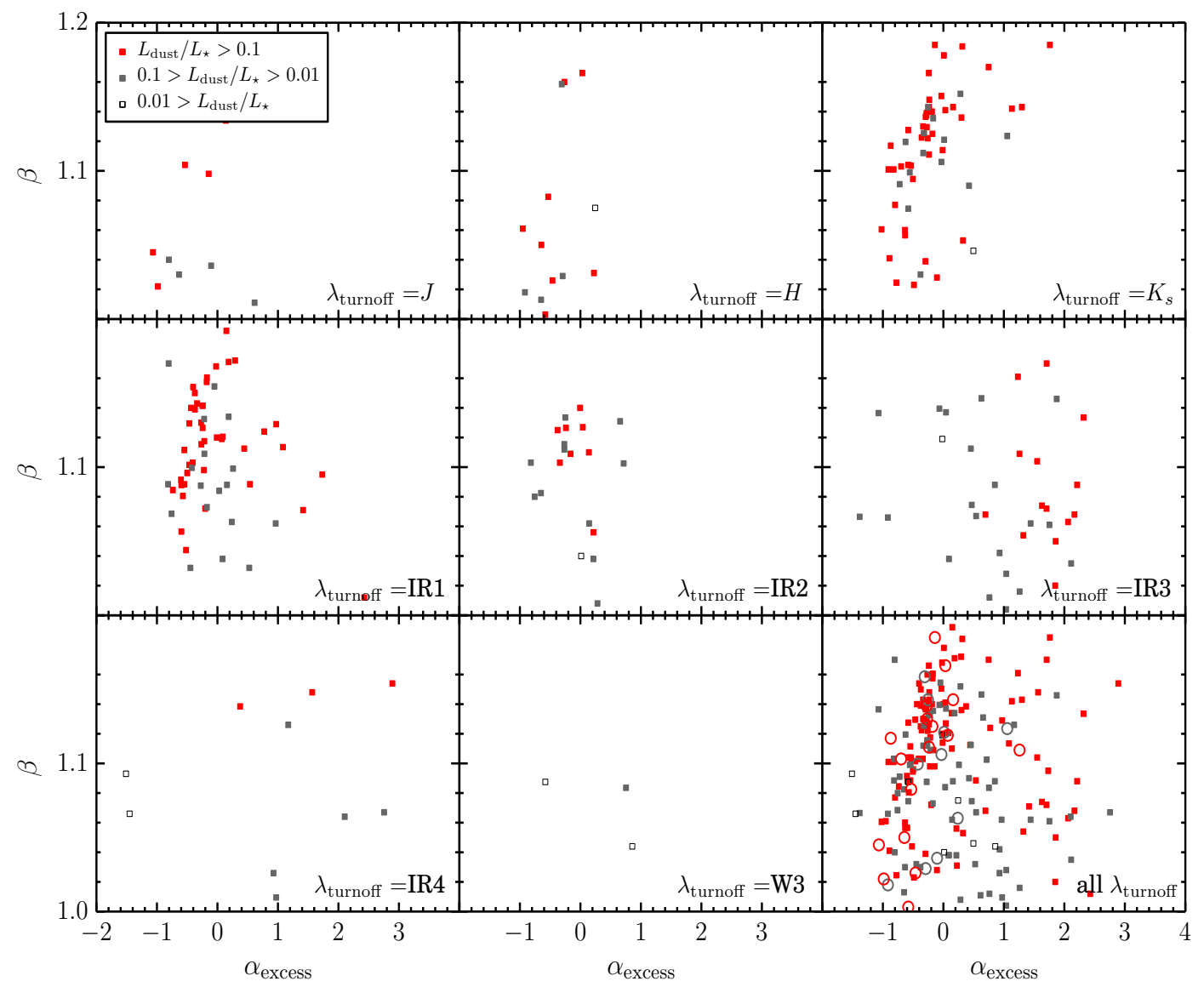

Fig. 12 Distribution of disk flaring power $\beta$ vs. $\alpha_{\text {excess }}$ for different $\lambda_{\text {turnoff }}$. As in Figure 11. YSOs with different ranges of $L_{\text {dust }} / L_{\star}$ are plotted with different symbols, as indicated in the top left panel. Distribution of the full sample is shown in the bottom right panel, where objects with $70 \mu \mathrm{m}$ detections are shown as red open circles.

\& Hartmann 1987; Chiang \& Goldreich 1997). The disk flaring power $\beta$ describes the radial gradient of the disk scale height $h$, i.e. $h(r) \propto r^{\beta}$, where $r$ is the cylindrical radius along the disk. Relationship between $\beta$ and $\alpha_{\text {excess }}$ for our YSOs is shown in Figure 12 While no significant correlation between $\beta$ and $\alpha_{\text {excess }}$ was found for the overall sample, a majority of the disks with $\alpha_{\text {excess }}<0.0$ follow a trend that $\alpha_{\text {excess }}$ increases with $\beta$, suggesting that the lack of a correlation between $R_{\text {in }} / R_{\text {sub }}$ and $\alpha_{\text {excess }}$ for small disk inner radii can be in part attributed to the disk flaring.

\subsection{Discussion}

A variety of physical mechanisms have been invoked to explain the circumstellar disk evolution and clearing processes (e.g. Henning \& Meeus 2011; Williams \& Cieza 2011). The few commonlyconsidered mechanisms include viscous disk accretion (e.g. Hartmann et al. 1998; Lynden-Bell \& Pringle 1974; Shakura \& Sunyaev 1973), grain growth and dust settling (e.g. Dullemond \& Dominik 2005; Tanaka et al. 2005), photoevaporative dispersal (e.g. Alexander et al. 2006a,b; Gorti \& Hollenbach 2009; Hollenbach et al. 1994; Shu et al. 1993; ) and dynamical clearing by companion stars or planets (e.g. Artymowicz \& Lubow 1994; Kley \& Nelson 2012; Lubow \& D’Angelo 2006; Zhu et al. 2012). 
While all of these proposed processes may operate simultaneously, it is important to probe the dominant process(es) at different disk evolution stages.

\subsubsection{From $\alpha_{\text {excess }}$ to Disk Geometry}

The near- to mid-IR $\alpha_{\text {excess }}$ is primarily affected by the inner disk clearing and outer disk flaring. In particular, the edge region of the optically thick inner disk, which is determined by either dust sublimation or some clearing processes, is illuminated directly by the stellar irradiation and thus contributes most of the hot dust emission excess, with the irradiation peak of this inner edge being shifted from near- to mid-IR as the disk is progressively cleared inside-out. In addition, as the disk evolves, dust settling or other clearing processes may result in a gradual reduction of disk flaring, which would in turn reduce the disk area that intercepts the stellar radiation and thus suppress the reprocessed cooler dust emission. Therefore, a progressively increasing disk inner edge is expected to increase $\alpha_{\text {excess }}$, whereas a smaller flaring power in the outer disk can result in a smaller $\alpha_{\text {excess }}$.

Our results suggest that variation of $\alpha_{\text {excess }}$ above $\sim 0.0$ primarily reflects the variation of disk clearing radii, whereas variation of $\alpha_{\text {excess }}$ below $\sim 0.0$ is largely related to a variation of the disk flaring power. Disk flaring is only important in shaping the near-to mid-IR SEDs when $R_{\text {in }} \lesssim 10 \times R_{\text {sub }}(>0.5$ AU for our sample). The lack of correlation between $\alpha_{\text {excess }}$ and disk flaring power at $R_{\text {in }} \gtrsim 10 \times R_{\text {sub }}$ implies that either the outer disk geometry does not vary synchronously with the inside-out disk clearing processes or spectral slopes at $\lambda \lesssim 24 \mu \mathrm{m}$ are not sensitive to the outer disk flaring. The small sample size of our disks (especially those with $\alpha_{\text {excess }}>0.0$ ) with detection at $70 \mu \mathrm{m}$ which is more sensitive to the outer disk flaring than shorter wavelengths (e.g. Sicilia-Aguilar et al. 2015) makes it hard to ascertain whether or not the outer disk flaring decreases or increases as the disk is cleared from the inside out. Recent studies of transitional disks in several nearby star-forming regions by Howard et al. (2013) and Keane et al. (2014) found that the continuum normalized [O I] $63.18 \mu \mathrm{m}$ line luminosities, which traces the cool, outer disks, are suppressed by a factor of $\sim 2$ on average with respect to the classical full disks, and this suppression was attributed to reduction of either the outer disk flaring or gas-to-dust ratio.

\subsubsection{Probing Disk Dispersal Processes with Transitional Disks}

There may be a variety of evolutionary paths from the optically thick full disks to optically thin to debris disks. Distinguishing different disk dispersal processes is crucial for understanding how the planetary systems formed from protoplanetary disks. The partially-cleared transitional disks, which have little or no excess emission in the near-IR $(\lesssim 5 \mu \mathrm{m})$ and thus optically thin inner opacity holes but a significant excess at longer wavelengths (e.g. Brown et al. 2007; Calvet et al. 2005; Strom et al. 1989; Skrutskie et al. 1990), provide a unique opportunity to probe different disk clearing mechanisms because different mechanisms are expected to result in very different IR spectral slopes, disk luminosities, and accretion activities in the short transitional stages (e.g. Alexander et al. 2014; Cieza et al. 2010; Najita, Strom \& Muzerolle 2007).

To open an inner opacity hole through photoevaporation, the disk viscous accretion rate has to fall below the photoevaporation rate (e.g. Alexander, Clarke \& Pringle 2006a; Owen et al. 2010), and once this happens, the full disks of gas and small dust grains can be quickly dissipated from the inside out in $\lesssim 0.1$ Myr which is an order of magnitude shorter than the typical disk lifetime. Besides a low fractional disk luminosity and steep IR spectral slope (e.g. $\alpha_{\text {excess }}<0.0$ ), another important consequence from photoevaporative clearing is that little or no accretion is expected once an inner hole is opened. In contrast, dynamical clearing by giant planets may sustain a small but still considerable amount of disk accretion across the inner opacity hole and a relatively high outer disk masses and luminosities and thus rising mid- to far-IR SEDs (e.g. Alexander 2008; Najita et al. 2007). In contrary to both photoevaporation and dynamical clearing, the pure grain growth and dust settling processes can result in an efficient depletion of small grains (and thus suppression of near- to mid-IR emission) from the inside out over time scales much smaller than 0.1 Myr (e.g. Dullemond \& Dominik 2005), with little direct influence on the accretion activity. 


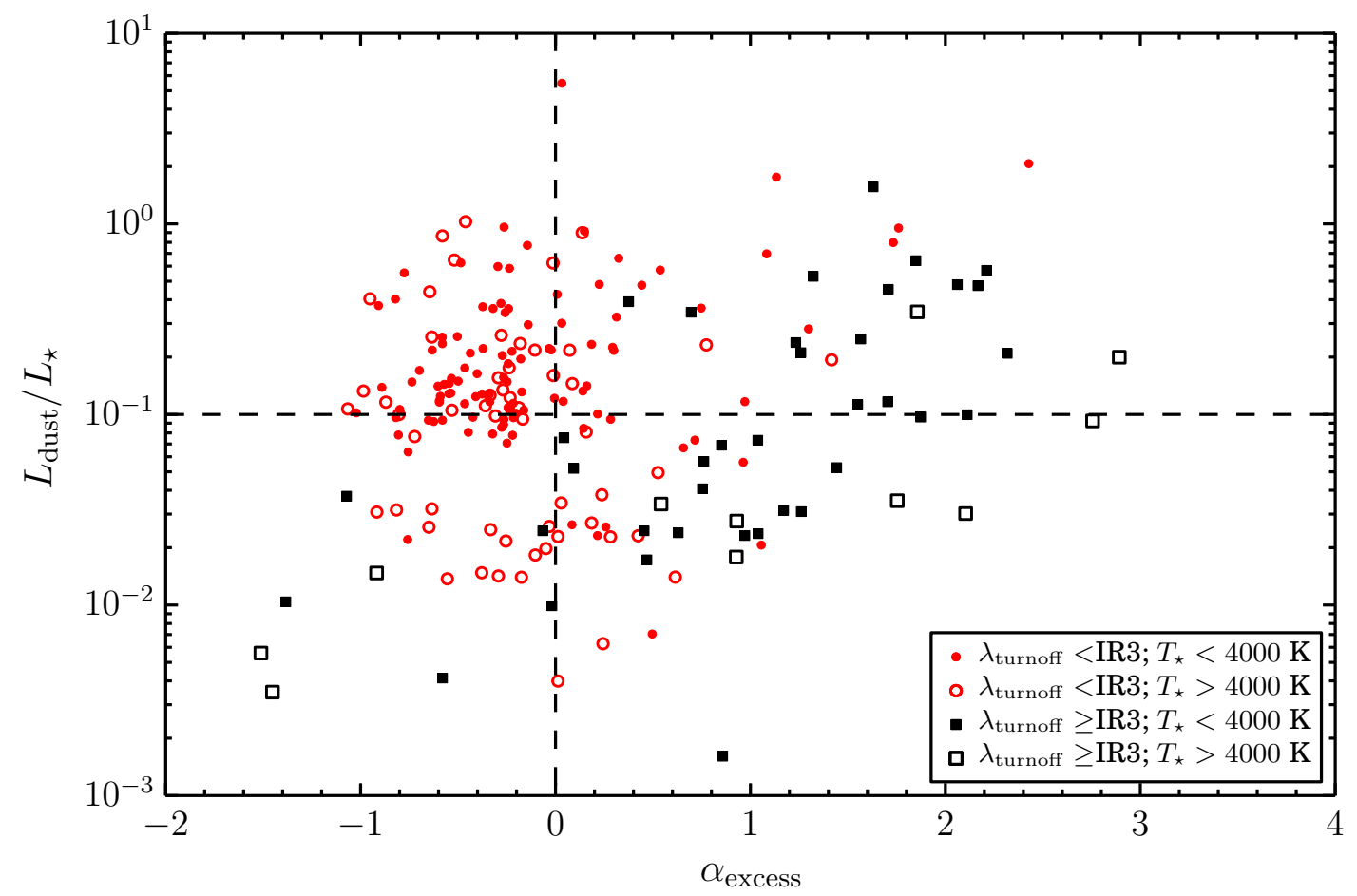

Fig. $13 \alpha_{\text {excess }}$ is plotted against the fractional disk luminosities $L_{\text {dust }} / L_{\star}$. Disks with $\lambda_{\text {turnoff }}<$ and $\geq$ IR3 (transitional disks) are plotted as red open circles and black filled squares respectively. The horizontal dashed line separates the sample into disks with $L_{\text {dust }} / L_{\star}>$ and $<0.1$, and the vertical dashed line separates the sample into disks with $\alpha_{\text {excess }}>$ and $<0.0$. Most accreting disks were found to have $L_{\text {dust }} / L_{\star} \geq 0.1$. Transitional disks in the lower left part may be primarily cleared by photoevaporation, while those in the upper right part may be dynamically cleared by giant planets.

All of our YSOs have $L_{\text {dust }} / L_{\star}>10^{-3}$, and $49(23 \%)$ have $\lambda_{\text {turnoff }} \geq$ IR3 and thus can be classified as transitional disks. Recall that for our sample disks with $\lambda_{\text {turnoff }} \geq$ IR3 exhibit a remarkably higher median and larger scatter of $\alpha_{\text {excess }}$ than those with $\lambda_{\text {turnoff }}<$ IR3 (Figure 9). The fraction of transitional disks in our sample is slightly higher yet still comparable to previous studies of nearby star clusters or star-forming regions (e.g. Currie et al. 2009; Dahm \& Carpenter 2009; Fang et al. 2009; Hernández et al. 2007; Kim et al. 2009; Lada et al. 2006). The distribution of our sample on the $\alpha_{\text {excess }}$ vs. $L_{\text {dust }} / L_{\star}$ plane is shown in Figure 13 where the transitional disks are plotted as black squares (filled for those with $T_{\star}<4000 \mathrm{~K}$, and open for those with $T_{\star}>4000 \mathrm{~K}$ ). Note that previous studies did not subtract the stellar photosphere emission for calculating the excess spectral index, which tends to underestimate the "genuine" $\alpha_{\text {excess }}$.

As is shown in Figure 13, the majority of the disks with $\lambda_{\text {turnoff }}<$ IR3 clustered toward the upper left corner, with $L_{\text {dust }} / L_{\star} \gtrsim 10^{-1}$ and $\alpha_{\text {excess }} \lesssim 0.0$, whereas the disks with $\lambda_{\text {turnoff }} \geq$ IR3 seem to follow a sequence from the upper right to the lower left, with none of them having $L_{\mathrm{dust}} / L_{\star}>10^{-1}$ and $\alpha_{\text {excess }}<0.0$. Most of the objects around the upper left corner are expected to have accreting full disks, and they are clearly separated from the population of transitional disks in Figure 13 A similar separation of transitional disks and full disks was also recently found by Sicilia-Aguilar et al. (2015) based on the relation between spectral indices and accretion rates. Among the objects with $\lambda_{\text {turnoff }}<$ IR3, $14(7 \%)$ have $\alpha_{\text {excess }}<0.0$ and $L_{\text {dust }} / L_{\star} \leq 0.003$. These $7 \%$ objects are consistent with being the 
so-called "anemic" (e.g. Lada et al. 2006) or "homologously depleted" (e.g. Currie \& Sicilia-Aguilar 2011) disks, which have detectable excess emission that decreases steadily at all wavelengths.

Transitional disks toward the lower left corner of Figure 13 may be more evolved than those toward the upper right. Among the 49 transitional disks, 41 (84\%) have $\alpha_{\text {excess }}>0.0$ and $8(16 \%)$ have $\alpha_{\text {excess }}<0.0$. Observations of UV continuum or recombination emission lines for all of our sample will be necessary for obtaining ongoing disk accretion rate. The accretion activities are known to be closely connected to the disk global properties, such as disk luminosities, masses and dust settling. If we instead use $L_{\text {dust }} / L_{\star}$ to approximately discriminate disks with and without accretion activity at a dividing value $=0.1,17(35 \%)$ of the 49 transitional disks have $\alpha_{\text {excess }}>0.0$ and $L_{\text {dust }} / L_{\star}>$ 0.1 , which may indicate the possibility of dynamical clearing by giant planets; Among the $32(65 \%)$ disks with $L_{\text {dust }} / L_{\star}<0.1,8$ have $\alpha_{\text {excess }}<0.0$ and 24 have $\alpha_{\text {excess }}>0.0$, the low $L_{\text {dust }} / L_{\star}$ probably indicates that these 32 disks are primarily cleared by photoevaporation. None of our transitional disks have $\alpha_{\text {excess }}<0.0$ and $L_{\text {dust }} / L_{\star}>0.1$, so grain growth and dust settling alone are probably not important hole-opening mechanisms (Ceiza et al. 2010). Furthermore, our finding that the median $\alpha_{\text {excess }}$ of Stages I and II YSOs tend to increases with $\lambda_{\text {turnoff }}$ also suggests that disk clearing is not primarily driven by grain growth which would otherwise results in a negative correlation between $\alpha_{\text {excess }}$ and $\lambda_{\text {turnoff }}$ (e.g. Dullemond \& Dominik 2005).

\section{SUMMARY}

We have statistically explored the properties of the central stellar sources, the evolutionary stages, and the circumstellar disks for a sample of 211 Perseus YSOs by modeling the optical to mid-IR broadband SEDs with the R06 YSO evolution models. The median central stellar mass and age for the Perseus YSOs are $\sim 0.3 M_{\odot}$ and $\sim 3.1$ Myr respectively based on the Siess et al. (2000) PMS evolutionary models. About $81 \%$ of our sample are classified as Stage II objects which are characterized by having optically thick disks, $\sim 14 \%$ are classified as Stage I objects which are characterized by having significant infalling envelopes, and the remaining 5\% are classified as Stage III objects with optically thin disks. Our primary results are summarized as follows.

- The evolutionary Stages as determined from the SED modeling have a general correspondence with the traditional spectral-indices-based Classes. In particular, $\sim 90 \%$ of the Class II YSOs fall into the Stage II phase which is characterized by optically thick disks, and 75\% of the Class I YSOs fall into the Stage I phase which is characterized by significant infalling envelopes. Nevertheless, relating the Classes III and FLAT YSOs to specific evolutionary stages is uncertain. In particular, half of the Class III YSOs fall into the Stage II and the other half fall into the optically thin Stage III phase, and half of the Class FLAT YSOs fall into the Stage I and the other half fall into the Stage II phase.

- We determined the turnoff wave band ( $\left.\lambda_{\text {turnoff }}\right)$ longward of which significant IR excesses with respect to the stellar photosphere level start to be observed and the excess spectral indices $\alpha_{\text {excess }}$

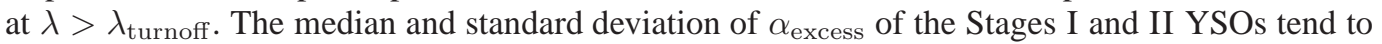
increase with $\lambda_{\text {turnoff }}$, especially at $\lambda_{\text {turnoff }} \geq$ IRAC $5.8 \mu \mathrm{m}$. There is a general trend that the median fractional dust luminosity $L_{\text {dust }} / L_{\star}$ decrease with increasing $\lambda_{\text {turnoff }}$, pointing to an inside-out disk clearing of small dust grains. We found a positive correlation between $\alpha_{\text {excess }}$ and disk inner radius $R_{\text {in }}$, and a lack of correlation between $\alpha_{\text {excess }}$ and disk flaring at $\alpha_{\text {excess }} \gtrsim 0.0$ and $R_{\text {in }} \gtrsim 10 \times R_{\text {sub }}$, which indicates that, firstly, the near- to mid-IR spectral slopes primarily reflect the progressive disk clearing from the inside out once $R_{\text {in }} \gtrsim 10 \times R_{\text {sub }}$, secondly, the outer disk flaring either does not vary synchronously with the inner disk clearing processes or has little appreciable influence on the spectral slopes at wavelengths $\lesssim 24 \mu \mathrm{m}$.

- About $23 \%$ (49) of our YSOs are classified as transitional disks, which have $\lambda_{\text {turnoff }} \geq$ IRAC $5.8 \mu \mathrm{m}$ and $L_{\text {dust }} / L_{\star}>10^{-3}$. By using the $L_{\text {dust }} / L_{\star}$ to approximately discriminate disks with and without accretion activity at a dividing value of $0.1,35 \%$ of the transitional disks have $\alpha_{\text {excess }}>0.0$ and $L_{\text {dust }} / L_{\star}>0.1$, implying the possibility of dynamical clearing by giant planets; $65 \%$ have 
$L_{\text {dust }} / L_{\star}<0.1$, which is consistent with the expectation of photoevaporative clearing; None of our disks has $\alpha_{\text {excess }}<0.0$ and $L_{\text {dust }} / L_{\star}>0.1$, so grain growth and dust settling are probably not the driven mechanisms in disk clearing, in line with the trend that the median $\alpha_{\text {excess }}$ increases, rather than decreases, with $\lambda_{\text {turnoff }}$.

An indispensable diagnostic of the evolutionary stages of YSOs and their circumstellar disks is the current accretion rate, which is usually determined either from recombination lines or ultraviolet continuum excesses. Different disk clearing processes can lead to different disk accretion properties, the effect of which is especially prominent in transitional stages. Moreover, similar to many previous studies, our current work is heavily biased against the Stage III YSOs with optically thin or anemic disks. To understand the disk evolution and dispersal processes, a systematic census of Stage III YSOs and their disk accretion activity is imperative. Therefore, our future direction will include 1) a systematic spectroscopic followup of our YSOs with the Large Sky Area Multi-Object Fiber Spectroscopic Telescope (LAMOST; Cui et al. 2012) to place stringent constraints on the on-going accretion activity; 2) a wide-field time-series optical photometry across the whole Perseus region for an unbiased census of Stage III disks with the PMO Xuyi 1.2-m Schmidt Telescope, in order to further probe the dominant disk dispersal mechanisms.

Table 1: Optical Photometry of Perseus YSOs

\begin{tabular}{|c|c|c|c|c|c|c|c|c|}
\hline$\overline{\mathrm{ID}_{\mathrm{c} 2 \mathrm{~d}}}$ & R.A.(J2000) & Decl.(J2000) & $g$ & $\sigma_{g}$ & $r$ & $\sigma_{r}$ & $i$ & $\sigma_{i}$ \\
\hline & $(\mathrm{deg})$ & $(\operatorname{deg})$ & (mag) & (mag) & (mag) & (mag) & (mag) & $(\mathrm{mag})$ \\
\hline \multicolumn{9}{|c|}{$J$ band turnoff ${ }^{3}$} \\
\hline $\mathrm{c} 2 \mathrm{dJ} 032852.2+304506$ & 52.21736 & 30.75154 & 13.96 & 0.06 & 12.9 & 0.06 & & \\
\hline c2dJ032854.6+311651 & 52.22763 & 31.28086 & & & 18.73 & 0.08 & 16.7 & 0.06 \\
\hline c2dJ032917.7+312245 & 52.32366 & 31.37917 & 15.55 & 0.06 & 13.82 & 0.05 & & \\
\hline c2dJ033035.5+311559 & 52.64781 & 31.26627 & 18.78 & 0.07 & 18.0 & 0.07 & 17.05 & 0.06 \\
\hline c2dJ033330.4+311051 & 53.37669 & 31.18071 & 15.99 & 0.05 & 13.98 & 0.06 & & \\
\hline c2dJ034157.4+314837 & 55.48934 & 31.81021 & 18.38 & 0.06 & 16.22 & 0.06 & 14.7 & 0.06 \\
\hline c2dJ034344.5+314309 & 55.93535 & 31.71926 & 18.69 & 0.06 & 17.19 & 0.06 & 14.52 & 0.06 \\
\hline c2dJ034413.0+320135 & 56.05407 & 32.02652 & & & & & 18.99 & 0.13 \\
\hline c2dJ034441.2+321010 & 56.17156 & 32.16944 & & & & & 17.4 & 0.07 \\
\hline \multicolumn{9}{|c|}{$H$ band turnoff } \\
\hline $\mathrm{c} 2 \mathrm{dJ} 032519.5+303424$ & 51.33134 & 30.57338 & 19.24 & 0.07 & 18.75 & 0.09 & 18.34 & 0.06 \\
\hline c2dJ033037.0+303128 & 52.65402 & 30.52437 & 20.41 & 0.11 & 17.82 & 0.06 & 15.82 & 0.06 \\
\hline c2dJ033044.0+303247 & 52.68326 & 30.54639 & 16.13 & 0.07 & 14.55 & 0.07 & 13.21 & 0.08 \\
\hline c2dJ033118.3+304940 & 52.82625 & 30.82765 & 18.11 & 0.06 & 15.31 & 0.06 & 14.48 & 0.06 \\
\hline c2dJ033312.8+312124 & 53.30349 & 31.35673 & & & 19.61 & 0.13 & 17.88 & 0.06 \\
\hline c2dJ033341.3+311341 & 53.42204 & 31.22806 & 20.9 & 0.32 & 18.52 & 0.07 & 17.73 & 0.06 \\
\hline c2dJ034109.1+314438 & 55.28804 & 31.74386 & 17.63 & 0.06 & 15.18 & 0.06 & 14.28 & 0.06 \\
\hline c2dJ034255.9+315842 & 55.73312 & 31.97834 & 14.69 & 0.06 & 13.45 & 0.06 & & \\
\hline c2dJ034426.7+320820 & 56.11124 & 32.13898 & 19.45 & 0.12 & 18.34 & 0.08 & 16.7 & 0.06 \\
\hline c2dJ034431.1+321848 & 56.12973 & 32.31347 & & & 19.86 & 0.37 & 18.09 & 0.08 \\
\hline c2dJ034437.9+320804 & 56.15785 & 32.13448 & 18.1 & 0.06 & 16.13 & 0.06 & 14.87 & 0.06 \\
\hline c2dJ034516.3+320620 & 56.31809 & 32.10559 & 18.33 & 0.06 & 16.19 & 0.06 & 14.85 & 0.06 \\
\hline c2dJ034520.5+320634 & 56.33525 & 32.10958 & 17.57 & 0.06 & 15.39 & 0.06 & 14.1 & 0.06 \\
\hline \multicolumn{9}{|c|}{$K_{s}$ band turnoff } \\
\hline $\mathrm{c} 2 \mathrm{dJ} 032741.5+302017$ & 51.92281 & 30.33799 & 16.46 & 0.06 & 14.74 & 0.06 & & \\
\hline c2dJ032800.1+300847 & 52.00038 & 30.1464 & 19.82 & 0.08 & 17.32 & 0.06 & 15.13 & 0.07 \\
\hline c2dJ032847.6+312406 & 52.19853 & 31.40168 & & & & & 19.64 & 0.11 \\
\hline c2dJ032850.6+304245 & 52.2109 & 30.7124 & & & & & 20.31 & 0.28 \\
\hline c2dJ032851.0+311818 & 52.21262 & 31.30513 & 18.49 & 0.07 & 15.81 & 0.07 & 14.0 & 0.07 \\
\hline c2dJ032851.2+311955 & 52.21335 & 31.3319 & 18.19 & 0.06 & 15.94 & 0.06 & 14.7 & 0.06 \\
\hline c2dJ032859.6+312147 & 52.24817 & 31.36296 & 18.42 & 0.06 & 16.13 & 0.06 & 15.59 & 0.06 \\
\hline c2dJ032903.8+311604 & 52.26574 & 31.26773 & 20.12 & 0.13 & 17.61 & 0.07 & 16.01 & 0.06 \\
\hline c2dJ032903.9+305630 & 52.26613 & 30.9416 & & & & & 18.87 & 0.07 \\
\hline c2dJ032903.9+312149 & 52.26614 & 31.3635 & 17.15 & 0.07 & 15.28 & 0.08 & 14.18 & 0.07 \\
\hline c2dJ032909.0+312624 & 52.28738 & 31.43997 & & & & & 19.95 & 0.17 \\
\hline
\end{tabular}

\footnotetext{
3 The YSOs start exhibiting significant ( $3 \sigma$ ) IR excesses above the photosphere level longward of the turnoff wavebands.
} 


\begin{tabular}{|c|c|c|c|c|c|c|c|c|}
\hline $\mathrm{ID}_{\mathrm{C} 2 \mathrm{~d}}$ & R.A.(J2000) & Decl.(J2000) & $g$ & $\sigma_{g}$ & $r$ & $\sigma_{r}$ & $i$ & $\sigma_{i}$ \\
\hline & $(\operatorname{deg})$ & (deg) & (mag) & (mag) & (mag) & (mag) & (mag) & (mag) \\
\hline c2dJ032910.8+311643 & 52.29515 & 31.27849 & & & 19.79 & 0.16 & 18.71 & 0.07 \\
\hline c2dJ032913.1+312253 & 52.30474 & 31.38134 & & & 19.71 & 0.19 & 17.3 & 0.07 \\
\hline c2dJ032921.9+311536 & 52.34115 & 31.26005 & 15.29 & 0.05 & 14.96 & 0.06 & 13.49 & 0.06 \\
\hline c2dJ032923.2+312030 & 52.34653 & 31.34173 & 18.59 & 0.06 & 16.9 & 0.06 & 15.14 & 0.06 \\
\hline c2dJ032932.6+312437 & 52.38573 & 31.41025 & & & 17.79 & 0.06 & 16.65 & 0.06 \\
\hline c2dJ033001.9+303529 & 52.5078 & 30.59145 & & & 19.09 & 0.1 & 18.21 & 0.08 \\
\hline c2dJ033035.9+303024 & 52.64968 & 30.50678 & & & 12.07 & 0.07 & & \\
\hline c2dJ033038.2+303212 & 52.65919 & 30.53665 & & & 19.7 & 0.2 & 18.44 & 0.07 \\
\hline c2dJ033052.5+305418 & 52.71878 & 30.90494 & & & 19.05 & 0.12 & 17.14 & 0.06 \\
\hline c2dJ033114.7+304955 & 52.81127 & 30.83206 & 18.53 & 0.06 & 16.91 & 0.06 & 15.8 & 0.06 \\
\hline c2dJ033142.4+310625 & 52.92668 & 31.10691 & 19.8 & 0.1 & 17.66 & 0.06 & 15.8 & 0.06 \\
\hline c2dJ033233.0+310222 & 53.13745 & 31.03935 & 20.13 & 0.11 & 17.84 & 0.06 & 15.38 & 0.06 \\
\hline c2dJ033234.0+310056 & 53.14185 & 31.01549 & 18.02 & 0.06 & 15.79 & 0.06 & 14.34 & 0.06 \\
\hline c2dJ033241.7+311046 & 53.17377 & 31.17953 & 20.63 & 0.2 & 18.35 & 0.07 & 16.65 & 0.06 \\
\hline c2dJ033401.7+311440 & 53.50692 & 31.24438 & 17.86 & 0.06 & 15.65 & 0.06 & 13.95 & 0.06 \\
\hline c2dJ033915.8+312431 & 54.81587 & 31.40854 & & & & & 18.77 & 0.07 \\
\hline c2dJ034119.2+320204 & 55.32994 & 32.03438 & 20.07 & 0.13 & 17.76 & 0.07 & 16.15 & 0.06 \\
\hline c2dJ034155.7+314811 & 55.48214 & 31.80318 & & & & & 20.11 & 0.12 \\
\hline c2dJ034157.8+314801 & 55.49064 & 31.80023 & & & 19.16 & 0.08 & 16.9 & 0.06 \\
\hline c2dJ034219.3+314327 & 55.5803 & 31.72415 & & & 19.56 & 0.1 & 17.44 & 0.06 \\
\hline c2dJ034232.9+314221 & 55.63711 & 31.70572 & & & 17.63 & 0.06 & 16.16 & 0.06 \\
\hline c2dJ034322.2+314614 & 55.84257 & 31.77045 & & & 19.32 & 0.11 & 17.45 & 0.06 \\
\hline c2dJ034328.2+320159 & 55.86753 & 32.0331 & 16.88 & 0.06 & 15.68 & 0.06 & 14.52 & 0.06 \\
\hline c2dJ034355.2+315532 & 55.98018 & 31.92559 & & & & & 18.9 & 0.08 \\
\hline c2dJ034356.0+320213 & 55.98346 & 32.03702 & 20.51 & 0.17 & 18.09 & 0.07 & 16.35 & 0.06 \\
\hline c2dJ034358.6+321728 & 55.99406 & 32.29097 & & & 16.32 & 0.1 & 14.93 & 0.07 \\
\hline c2dJ034358.9+321127 & 55.99549 & 32.19088 & 18.06 & 0.06 & 16.36 & 0.06 & 15.3 & 0.05 \\
\hline c2dJ034359.9+320441 & 55.9995 & 32.07817 & & & & & 17.67 & 0.06 \\
\hline c2dJ034406.0+321532 & 56.02504 & 32.25892 & & & & & 17.74 & 0.07 \\
\hline c2dJ034406.8+320754 & 56.02833 & 32.13167 & 19.46 & 0.1 & 17.81 & 0.07 & 16.1 & 0.06 \\
\hline c2dJ034407.5+320409 & 56.03132 & 32.0691 & 20.37 & 0.17 & 18.28 & 0.08 & 16.48 & 0.06 \\
\hline c2dJ034411.6+320313 & 56.04844 & 32.05364 & & & 19.03 & 0.17 & 17.22 & 0.06 \\
\hline c2dJ034418.6+321253 & 56.07747 & 32.21475 & & & 18.66 & 0.1 & 19.08 & 0.13 \\
\hline c2dJ034421.6+321038 & 56.0901 & 32.17713 & 18.99 & 0.08 & 16.85 & 0.06 & 15.72 & 0.06 \\
\hline c2dJ034422.3+321201 & 56.09307 & 32.20019 & 18.31 & 0.06 & 16.67 & 0.06 & 15.26 & 0.06 \\
\hline c2dJ034425.5+321131 & 56.10633 & 32.192 & 19.28 & 0.08 & 17.22 & 0.06 & 15.63 & 0.06 \\
\hline c2dJ034427.3+321421 & 56.11359 & 32.23915 & 19.42 & 0.08 & & & 16.0 & 0.06 \\
\hline c2dJ034431.4+320014 & 56.13069 & 32.00394 & & & & & 18.56 & 0.08 \\
\hline c2dJ034435.7+320304 & 56.1487 & 32.05097 & 20.47 & 0.2 & 18.88 & 0.13 & 17.55 & 0.06 \\
\hline c2dJ034438.5+320736 & 56.16024 & 32.12659 & 16.84 & 0.06 & 15.25 & 0.06 & 14.38 & 0.06 \\
\hline c2dJ034438.5+320801 & 56.1606 & 32.13351 & 18.92 & 0.07 & 16.9 & 0.06 & 15.21 & 0.06 \\
\hline c2dJ034444.7+320402 & 56.18633 & 32.06736 & 17.54 & 0.06 & 15.42 & 0.06 & 14.06 & 0.06 \\
\hline c2dJ034452.0+322625 & 56.21668 & 32.4404 & & & 18.83 & 0.1 & 16.92 & 0.06 \\
\hline c2dJ034452.1+315825 & 56.21689 & 31.97367 & & & & & 17.71 & 0.06 \\
\hline c2dJ034525.1+320930 & 56.35479 & 32.15842 & 19.0 & 0.07 & 16.85 & 0.06 & 15.06 & 0.06 \\
\hline c2dJ034536.8+322557 & 56.40347 & 32.43251 & 15.96 & 0.06 & 13.79 & 0.05 & & \\
\hline c2dJ034548.3+322412 & 56.45111 & 32.40334 & & & & & 10.71 & 0.07 \\
\hline c2dJ034558.2+322647 & 56.49269 & 32.44653 & 20.7 & 0.29 & 18.72 & 0.1 & 16.73 & 0.06 \\
\hline \multicolumn{9}{|c|}{ IR1 band turnoff } \\
\hline c2dJ032747.7+301205 & 51.94864 & 30.20126 & & & 18.17 & 0.07 & 16.14 & 0.07 \\
\hline c2dJ032834.5+310051 & 52.1437 & 31.01419 & & & & & 18.38 & 0.11 \\
\hline c2dJ032842.4+302953 & 52.17673 & 30.4981 & 17.97 & 0.06 & 16.28 & 0.06 & 14.53 & 0.06 \\
\hline c2dJ032844.1+312053 & 52.18372 & 31.34799 & & & & & 18.34 & 0.06 \\
\hline c2dJ032846.2+311638 & 52.19252 & 31.27734 & 16.63 & 0.06 & 14.93 & 0.06 & 13.48 & 0.06 \\
\hline c2dJ032847.8+311655 & 52.19933 & 31.28196 & 20.02 & 0.12 & 19.03 & 0.08 & 16.6 & 0.06 \\
\hline c2dJ032852.2+312245 & 52.2174 & 31.37924 & 17.9 & 0.06 & 15.99 & 0.06 & 14.64 & 0.06 \\
\hline c2dJ032856.6+311836 & 52.23602 & 31.30987 & & & 17.65 & 0.07 & 16.28 & 0.06 \\
\hline c2dJ032857.0+311622 & 52.23736 & 31.27285 & & & & & 18.12 & 0.07 \\
\hline c2dJ032903.1+312238 & 52.26311 & 31.37723 & 20.11 & 0.14 & 18.47 & 0.07 & 17.06 & 0.06 \\
\hline c2dJ032904.1+305613 & 52.26716 & 30.9369 & 20.58 & 0.16 & 19.41 & 0.12 & 17.06 & 0.06 \\
\hline c2dJ032918.7+312325 & 52.32808 & 31.39038 & 16.0 & 0.05 & 14.15 & 0.06 & 13.57 & 0.06 \\
\hline c2dJ032920.4+311834 & 52.33515 & 31.3095 & & & & & 18.72 & 0.13 \\
\hline
\end{tabular}




\begin{tabular}{|c|c|c|c|c|c|c|c|c|}
\hline $\mathrm{ID}_{\mathrm{C} 2 \mathrm{~d}}$ & R.A.(J2000) & Decl.(J2000) & $g$ & $\sigma_{g}$ & $r$ & $\sigma_{r}$ & $i$ & $\sigma_{i}$ \\
\hline & $(\operatorname{deg})$ & $(\operatorname{deg})$ & (mag) & (mag) & $(\mathrm{mag})$ & (mag) & (mag) & $(\mathrm{mag})$ \\
\hline $\mathrm{c} 2 \mathrm{dJ} 032930.4+311903$ & 52.37668 & 31.31759 & 17.57 & 0.06 & 16.05 & 0.06 & 14.69 & 0.06 \\
\hline c2dJ032932.9+312713 & 52.387 & 31.45349 & 19.35 & 0.07 & 17.67 & 0.06 & 15.93 & 0.06 \\
\hline c2dJ032937.7+312202 & 52.40723 & 31.36735 & & & 19.71 & 0.12 & 17.3 & 0.06 \\
\hline c2dJ032954.0+312053 & 52.47518 & 31.34803 & 17.81 & 0.06 & 15.8 & 0.06 & 14.72 & 0.06 \\
\hline c2dJ033024.1+311404 & 52.60034 & 31.23454 & 19.3 & 0.07 & 18.0 & 0.06 & 16.18 & 0.06 \\
\hline c2dJ033110.7+304941 & 52.79451 & 30.82795 & 18.55 & 0.06 & 15.52 & 0.06 & 15.05 & 0.06 \\
\hline c2dJ033430.8+311324 & 53.62826 & 31.22343 & & & 18.45 & 0.07 & 16.38 & 0.06 \\
\hline c2dJ033449.8+311550 & 53.70768 & 31.26396 & 15.85 & 0.06 & 14.11 & 0.06 & & \\
\hline c2dJ034001.5+311017 & 55.00621 & 31.17147 & & & 19.56 & 0.15 & 17.65 & 0.06 \\
\hline c2dJ034201.0+314913 & 55.50422 & 31.82038 & & & & & 19.16 & 0.07 \\
\hline c2dJ034204.3+314712 & 55.51807 & 31.78655 & & & & & 18.05 & 0.06 \\
\hline c2dJ034220.3+320531 & 55.58467 & 32.09195 & 20.03 & 0.12 & 17.57 & 0.06 & 16.14 & 0.06 \\
\hline c2dJ034232.1+315250 & 55.63377 & 31.88043 & & & & & 19.92 & 0.14 \\
\hline c2dJ034249.2+315011 & 55.70492 & 31.83643 & & & 19.47 & 0.1 & 17.11 & 0.06 \\
\hline c2dJ034313.7+320045 & 55.80708 & 32.01254 & & & & & 18.71 & 0.08 \\
\hline c2dJ034323.6+321226 & 55.84821 & 32.20718 & & & 19.32 & 0.12 & 17.23 & 0.06 \\
\hline c2dJ034329.4+315219 & 55.87265 & 31.87207 & & & & & 19.38 & 0.1 \\
\hline c2dJ034345.2+320359 & 55.9382 & 32.06628 & & & & & 18.51 & 0.1 \\
\hline c2dJ034348.8+321552 & 55.95345 & 32.26431 & 19.57 & 0.13 & 17.63 & 0.07 & 15.95 & 0.06 \\
\hline c2dJ034355.3+320753 & 55.98033 & 32.13147 & & & 19.01 & 0.13 & 17.04 & 0.06 \\
\hline c2dJ034359.1+321421 & 55.99624 & 32.23923 & 20.15 & 0.13 & 17.61 & 0.07 & 16.73 & 0.06 \\
\hline c2dJ034401.6+322359 & 56.00656 & 32.39968 & & & 18.68 & 0.11 & 16.73 & 0.06 \\
\hline c2dJ034402.9+315228 & 56.01215 & 31.87437 & & & & & 18.9 & 0.09 \\
\hline c2dJ034418.2+320457 & 56.0757 & 32.08249 & & & 18.09 & 0.07 & 16.4 & 0.06 \\
\hline c2dJ034425.5+320617 & 56.10645 & 32.10476 & & & 18.47 & 0.09 & 16.77 & 0.06 \\
\hline c2dJ034426.0+320430 & 56.10848 & 32.07512 & 16.19 & 0.06 & 14.19 & 0.06 & 13.33 & 0.06 \\
\hline c2dJ034427.9+322719 & 56.11625 & 32.45525 & 19.42 & 0.08 & 17.99 & 0.07 & 16.06 & 0.06 \\
\hline c2dJ034428.5+315954 & 56.1188 & 31.99833 & 18.65 & 0.06 & 16.69 & 0.06 & 15.03 & 0.06 \\
\hline c2dJ034429.8+320055 & 56.12418 & 32.01516 & & & & & 17.49 & 0.06 \\
\hline c2dJ034432.0+321144 & 56.1335 & 32.19548 & 15.47 & 0.06 & 13.8 & 0.06 & 12.77 & 0.06 \\
\hline c2dJ034433.8+315830 & 56.1408 & 31.97506 & & & 18.75 & 0.12 & 16.88 & 0.06 \\
\hline c2dJ034435.0+321531 & 56.1458 & 32.25865 & & & 18.36 & 0.08 & 16.5 & 0.06 \\
\hline c2dJ034435.5+320856 & 56.14779 & 32.14897 & & & & & 16.9 & 0.06 \\
\hline c2dJ034437.0+320645 & 56.15399 & 32.11256 & & & 12.16 & 0.06 & & \\
\hline c2dJ034437.4+320901 & 56.1559 & 32.15024 & & & 16.75 & 0.09 & 15.54 & 0.06 \\
\hline c2dJ034438.0+320330 & 56.15825 & 32.05825 & 17.72 & 0.06 & 15.43 & 0.06 & 14.14 & 0.06 \\
\hline c2dJ034439.8+321804 & 56.16583 & 32.30112 & 19.6 & 0.09 & 17.12 & 0.06 & 15.44 & 0.06 \\
\hline c2dJ034440.2+320933 & 56.16771 & 32.15917 & & & & & 17.01 & 0.08 \\
\hline c2dJ034442.6+321002 & 56.17741 & 32.16735 & & & & & 17.97 & 0.07 \\
\hline c2dJ034443.1+313734 & 56.17942 & 31.62603 & & & & & 19.06 & 0.08 \\
\hline c2dJ034443.8+321030 & 56.18241 & 32.1751 & 20.86 & 0.24 & 18.55 & 0.1 & 15.89 & 0.06 \\
\hline c2dJ034450.4+315236 & 56.20979 & 31.87667 & & & & & 18.27 & 0.09 \\
\hline c2dJ034456.1+320915 & 56.23394 & 32.15422 & 17.08 & 0.06 & 14.75 & 0.06 & 13.77 & 0.05 \\
\hline c2dJ034517.8+321206 & 56.32426 & 32.20162 & & & 18.78 & 0.09 & 16.78 & 0.06 \\
\hline c2dJ034529.7+315920 & 56.37382 & 31.98881 & & & & & 18.53 & 0.08 \\
\hline c2dJ034533.5+314555 & 56.38945 & 31.76536 & & & & & 19.73 & 0.13 \\
\hline c2dJ034535.6+315954 & 56.39849 & 31.99845 & & & & & 17.88 & 0.07 \\
\hline c2dJ034657.4+324917 & 56.7391 & 32.8215 & & & 18.7 & 0.09 & 16.86 & 0.06 \\
\hline \multicolumn{9}{|c|}{ IR2 band turnoff } \\
\hline $\mathrm{c} 2 \mathrm{dJ} 032851.1+311632$ & 52.21281 & 31.27566 & 19.99 & 0.08 & 18.22 & 0.07 & 16.27 & 0.06 \\
\hline c2dJ032852.2+311547 & 52.2173 & 31.26307 & & & 19.19 & 0.1 & 16.83 & 0.06 \\
\hline c2dJ032852.9+311626 & 52.22052 & 31.274 & 20.38 & 0.15 & 18.47 & 0.07 & 16.53 & 0.06 \\
\hline c2dJ032909.5+312721 & 52.28954 & 31.45581 & & & 20.12 & 0.23 & 17.96 & 0.06 \\
\hline c2dJ032917.8+311948 & 52.32406 & 31.33001 & & & & & 19.35 & 0.08 \\
\hline c2dJ032921.6+312110 & 52.33988 & 31.35287 & 18.91 & 0.07 & 16.85 & 0.06 & 15.24 & 0.06 \\
\hline c2dJ032923.2+312653 & 52.34687 & 31.44808 & 20.04 & 0.11 & 18.57 & 0.07 & 16.69 & 0.06 \\
\hline c2dJ032928.9+305842 & 52.37034 & 30.9783 & 18.75 & 0.06 & 17.2 & 0.06 & 15.42 & 0.06 \\
\hline c2dJ032929.8+312103 & 52.37415 & 31.35072 & 19.65 & 0.1 & 17.72 & 0.06 & 15.83 & 0.06 \\
\hline c2dJ032937.6+310249 & 52.40678 & 31.04699 & & & & & 17.85 & 0.06 \\
\hline c2dJ033120.1+304918 & 52.83379 & 30.82157 & & & 19.16 & 0.09 & 17.07 & 0.06 \\
\hline c2dJ033346.9+305350 & 53.44552 & 30.89726 & 20.43 & 0.17 & 18.4 & 0.07 & 16.55 & 0.06 \\
\hline c2dJ034336.0+315009 & 55.90007 & 31.83583 & & & & & 19.66 & 0.09 \\
\hline
\end{tabular}




\begin{tabular}{|c|c|c|c|c|c|c|c|c|}
\hline $\mathrm{ID}_{\mathrm{C} 2 \mathrm{~d}}$ & R.A.(J2000) & Decl.(J2000) & $g$ & $\sigma_{g}$ & $r$ & $\sigma_{r}$ & $i$ & $\sigma_{i}$ \\
\hline & $(\mathrm{deg})$ & $(\mathrm{deg})$ & (mag) & (mag) & (mag) & (mag) & (mag) & $(\mathrm{mag})$ \\
\hline c2dJ034346.5+321106 & 55.94357 & 32.18498 & & & & & 18.76 & 0.09 \\
\hline c2dJ034347.6+320903 & 55.94853 & 32.1507 & & & & & 18.54 & 0.09 \\
\hline c2dJ034415.8+315937 & 56.06598 & 31.99354 & & & & & 17.52 & 0.06 \\
\hline c2dJ034421.3+321156 & 56.08878 & 32.19897 & 19.09 & 0.07 & 16.94 & 0.06 & 15.49 & 0.06 \\
\hline c2dJ034427.2+322029 & 56.11342 & 32.34133 & & & & & 17.01 & 0.06 \\
\hline c2dJ034430.8+320956 & 56.12848 & 32.16547 & 11.93 & 0.08 & & & 11.02 & 0.15 \\
\hline c2dJ034658.5+324659 & 56.74379 & 32.78303 & & & 19.22 & 0.13 & 17.05 & 0.06 \\
\hline \multicolumn{9}{|c|}{ IR3 band turnoff } \\
\hline c2dJ032858.1+311804 & 52.24213 & 31.30102 & 19.06 & 0.07 & 16.92 & 0.06 & 15.65 & 0.06 \\
\hline c2dJ032908.0+312251 & 52.28315 & 31.38095 & & & 19.26 & 0.11 & 17.22 & 0.08 \\
\hline c2dJ032912.9+312329 & 52.30381 & 31.39147 & & & 18.36 & 0.09 & 16.65 & 0.06 \\
\hline c2dJ032916.8+312325 & 52.32013 & 31.39031 & & & & & 18.22 & 0.06 \\
\hline c2dJ032926.8+312648 & 52.36172 & 31.44654 & 15.81 & 0.05 & 14.11 & 0.06 & & \\
\hline c2dJ032929.3+311835 & 52.37198 & 31.30963 & 19.27 & 0.07 & 17.01 & 0.06 & 15.6 & 0.06 \\
\hline c2dJ034233.1+315215 & 55.63803 & 31.87075 & & & & & 18.86 & 0.08 \\
\hline c2dJ034234.2+315101 & 55.64244 & 31.85028 & & & & & 18.23 & 0.07 \\
\hline c2dJ034250.9+314045 & 55.71208 & 31.67921 & & & & & 18.44 & 0.06 \\
\hline c2dJ034301.9+314436 & 55.75807 & 31.74322 & & & & & 19.4 & 0.08 \\
\hline c2dJ034308.7+315139 & 55.78628 & 31.86072 & & & & & 19.06 & 0.08 \\
\hline c2dJ034344.6+320818 & 55.93594 & 32.13827 & 17.3 & 0.06 & 15.46 & 0.06 & 14.46 & 0.05 \\
\hline c2dJ034410.1+320405 & 56.0422 & 32.06792 & & & 19.2 & 0.16 & 16.93 & 0.06 \\
\hline c2dJ034415.2+321942 & 56.06348 & 32.32838 & & & & & 17.6 & 0.08 \\
\hline c2dJ034418.2+320959 & 56.07588 & 32.16648 & & & 19.23 & 0.16 & 17.14 & 0.06 \\
\hline c2dJ034422.3+320543 & 56.09287 & 32.09521 & 18.94 & 0.07 & 16.87 & 0.06 & 15.61 & 0.06 \\
\hline c2dJ034422.6+320154 & 56.09409 & 32.03157 & 18.87 & 0.07 & 16.75 & 0.06 & 15.05 & 0.06 \\
\hline c2dJ034425.7+321549 & 56.10713 & 32.26367 & & & & & 18.83 & 0.14 \\
\hline c2dJ034429.2+320116 & 56.1218 & 32.02103 & & & & & 18.9 & 0.1 \\
\hline c2dJ034429.7+321040 & 56.12391 & 32.17772 & 17.39 & 0.11 & 15.87 & 0.07 & 14.83 & 0.06 \\
\hline c2dJ034434.1+321636 & 56.14225 & 32.2766 & & & & & 17.57 & 0.06 \\
\hline c2dJ034434.8+315655 & 56.14503 & 31.94866 & 19.52 & 0.09 & 17.47 & 0.07 & 15.83 & 0.06 \\
\hline c2dJ034437.4+321224 & 56.15584 & 32.20671 & & & 18.47 & 0.09 & 16.92 & 0.06 \\
\hline c2dJ034438.0+321137 & 56.15838 & 32.19361 & & & 18.3 & 0.08 & 16.45 & 0.06 \\
\hline c2dJ034439.0+320320 & 56.16238 & 32.05547 & & & 19.05 & 0.18 & 17.39 & 0.06 \\
\hline c2dJ034439.2+322009 & 56.16331 & 32.3358 & 19.89 & 0.11 & 17.87 & 0.06 & 15.83 & 0.06 \\
\hline c2dJ034441.7+321202 & 56.17392 & 32.20062 & & & & & 16.98 & 0.06 \\
\hline c2dJ034442.6+320619 & 56.17758 & 32.10541 & 17.01 & 0.06 & 15.4 & 0.06 & 14.58 & 0.06 \\
\hline c2dJ034443.0+321560 & 56.17929 & 32.26656 & & & & & 18.15 & 0.07 \\
\hline c2dJ034444.6+320813 & 56.18579 & 32.13681 & 19.53 & 0.13 & 17.8 & 0.06 & 16.16 & 0.06 \\
\hline c2dJ034457.9+320402 & 56.24106 & 32.0671 & & & 18.63 & 0.1 & 16.56 & 0.06 \\
\hline c2dJ034460.0+322233 & 56.24997 & 32.37576 & & & & & 17.76 & 0.06 \\
\hline c2dJ034501.4+320502 & 56.25595 & 32.08382 & 13.76 & 0.06 & 12.79 & 0.05 & & \\
\hline c2dJ034504.7+321501 & 56.2694 & 32.2503 & & & 19.27 & 0.15 & 17.14 & 0.06 \\
\hline c2dJ034513.5+322435 & 56.30627 & 32.40966 & & & & & 17.89 & 0.06 \\
\hline \multicolumn{9}{|c|}{ IR4 band turnoff } \\
\hline c2dJ032854.1+311654 & 52.22537 & 31.28172 & & & 18.89 & 0.08 & 16.59 & 0.06 \\
\hline c2dJ033027.1+302830 & 52.61309 & 30.47493 & & & & & 18.39 & 0.1 \\
\hline c2dJ034227.1+314433 & 55.613 & 31.74247 & & & 19.17 & 0.11 & 17.33 & 0.06 \\
\hline c2dJ034254.7+314345 & 55.72778 & 31.72924 & 16.22 & 0.05 & 14.51 & 0.06 & 13.59 & 0.06 \\
\hline c2dJ034306.8+314821 & 55.77822 & 31.80569 & 20.05 & 0.12 & 17.63 & 0.06 & 15.63 & 0.06 \\
\hline c2dJ034419.1+320931 & 56.07973 & 32.15869 & & & & & 11.3 & 0.08 \\
\hline c2dJ034421.6+321510 & 56.08987 & 32.25271 & 18.57 & 0.1 & 17.62 & 0.07 & 15.83 & 0.06 \\
\hline c2dJ034431.5+320845 & 56.13145 & 32.14581 & 14.71 & 0.06 & 13.4 & 0.06 & 12.76 & 0.06 \\
\hline c2dJ034456.8+315411 & 56.23684 & 31.90317 & & & & & 17.45 & 0.06 \\
\hline c2dJ034507.6+321028 & 56.28182 & 32.17441 & & & 12.11 & 0.05 & & \\
\hline \multicolumn{9}{|c|}{ W3 band turnoff } \\
\hline c2dJ032916.7+311618 & 52.31955 & 31.27171 & 17.17 & 0.06 & 15.52 & 0.06 & 13.97 & 0.06 \\
\hline c2dJ033026.0+310218 & 52.60821 & 31.03831 & 15.27 & 0.05 & 13.72 & 0.05 & & \\
\hline c2dJ033351.1+311228 & 53.46281 & 31.20772 & 19.35 & 0.07 & 17.03 & 0.06 & 14.77 & 0.06 \\
\hline c2dJ034011.8+315523 & 55.04929 & 31.92315 & 19.42 & 0.07 & 17.39 & 0.06 & 16.17 & 0.06 \\
\hline
\end{tabular}

Notes: All the photometry was calibrated against the SDSS DR8 catalog. 
Table 2: SED Fitting Results of Perseus YSOs

\begin{tabular}{|c|c|c|c|c|c|c|c|c|c|c|c|c|}
\hline $\mathrm{ID}_{\mathrm{c} 2 \mathrm{~d}}$ & ID $_{\text {model }}$ & $\chi_{r}^{2}$ & $A_{V}$ & $D$ & $M_{\star}$ & $\mathrm{Age}_{\star}$ & $L_{\star}$ & $L_{\text {dust }}$ & $R_{\text {in }} / R_{\text {sub }}$ & Stage & $\alpha_{\text {turnoff }}$ & $\alpha_{\text {excess }}$ \\
\hline (1) & (2) & (3) & $\begin{array}{l}\text { (mag) } \\
\text { (4) }\end{array}$ & $\begin{array}{l}(\mathrm{kpc}) \\
(5)\end{array}$ & $\begin{array}{l}\left(M_{\odot}\right) \\
(6)\end{array}$ & $\begin{array}{l}\left(10^{5} \mathrm{yr}\right) \\
(7)\end{array}$ & $\begin{array}{l}\left(10^{-2} L_{\odot}\right) \\
(8)\end{array}$ & $\begin{array}{l}\left(10^{-2} L_{\odot}\right) \\
(9)\end{array}$ & (10) & (11) & (12) & (13) \\
\hline \multicolumn{13}{|c|}{$J$ band turnoff $4^{4}$} \\
\hline $\mathrm{c} 2 \mathrm{dJ} 032852.2+304506$ & 3000104 & 2.0 & 3.76 & 0.28 & $2.36_{-0.22}^{+0.45}$ & $76.39_{-23.22}^{+20.74}$ & 5439.09 & 173.77 & $1.7_{-0.7}^{+0.7}$ & II & -0.75 & -0.63 \\
\hline c2dJ032854.6+311651 & 3013176 & 0.4 & 3.04 & 0.23 & $0.14_{-0.04}^{+0.23}$ & $25.55_{-21.9}^{+27.4}$ & 8.72 & 1.13 & $4.2_{-3.2}^{+1.4}$ & II & -0.57 & -0.54 \\
\hline c2dJ032917.7+312245 & 3005510 & 0.5 & 5.7 & 0.26 & $2.22_{-1.11}^{+0.93}$ & $76.42_{-66.3}^{+22.48}$ & 2637.26 & 263.42 & $1.1_{-0.1}^{+0.5}$ & II & -0.95 & -0.8 \\
\hline c2dJ033035.5+311559 & 3018343 & 6.7 & 0.79 & 0.24 & $1.02_{-0.71}^{+1.83}$ & $8.92_{-8.84}^{+31.75}$ & 2475.96 & 1910.17 & $83.9_{-82.9}^{+237.7}$ & I & -0.46 & -0.14 \\
\hline c2dJ033330.4+311051 & 3003655 & 0.3 & 6.6 & 0.21 & $2.24_{-0.26}^{+0.51}$ & $62.4_{-26.81}^{+3.04}$ & 2561.06 & 339.83 & $1.0_{-0.0}^{+-0.0}$ & II & -0.8 & -0.99 \\
\hline c2dJ034157.4+314837 & 3015603 & 1.3 & 7.69 & 0.28 & $2.08_{-0.1}^{+0.13}$ & $93.61_{-4.8}^{+5.3}$ & 1964.93 & 210.13 & $1.0_{-0.0}^{+0.0}$ & II & -0.86 & -1.07 \\
\hline c2dJ034344.5+314309 & 3017083 & 2.5 & 3.27 & 0.28 & $3.11_{-0.15}^{+0.3}$ & $16.84_{-3.08}^{+59.29}$ & 6740.19 & 123.32 & $1.5_{-0.5}^{+0.5}$ & II & -0.13 & -0.1 \\
\hline c2dJ034413.0+320135 & 3017363 & 4.0 & 5.47 & 0.23 & $3.44_{-0.52}^{+0.156}$ & $42.96_{-17.21}^{+21.51}$ & 17530.01 & 245.48 & $1.5_{-0.5}^{+1.5}$ & II & 0.71 & 0.61 \\
\hline c2dJ034441.2+321010 & 3007882 & 1.2 & 4.09 & 0.3 & $0.9_{-0.63}^{+2.67}$ & $0.43_{-0.39}^{+2.08}$ & 3965.79 & 3561.31 & $1.3_{-0.3}^{+397.9}$ & I & -0.13 & 0.14 \\
\hline \multicolumn{13}{|c|}{$H$ band turnoff } \\
\hline c2dJ032519.5+303424 & 3005058 & 5.5 & 0.74 & 0.27 & $1.72_{-0.06}^{+0.06}$ & $40.9_{-14.26}^{+13.71}$ & 311.13 & 4.52 & $1.0_{-0.0}^{+0.0}$ & II & 0.04 & 0.16 \\
\hline c2dJ033037.0+303128 & 3000055 & 1.7 & 8.57 & 0.26 & $1.49_{-0.75}^{+0.9}$ & $26.92_{-13.42}^{+63.88}$ & 3108.59 & 95.56 & $1.1_{-0.1}^{+0.0}$ & III & -0.76 & -0.92 \\
\hline c2dJ033044.0+303247 & 3015197 & 1.4 & 3.81 & 0.24 & $0.8_{-0.47}^{+2.34}$ & $6.16_{-3.64}^{+84.64}$ & 142.18 & 145.99 & $1.2_{-0.2}^{+5.6}$ & II & -0.58 & -0.46 \\
\hline c2dJ033118.3+304940 & 3002814 & 2.0 & 5.21 & 0.25 & $\begin{array}{l}1.49_{-0.74}^{+1.1 .15} \\
\end{array}$ & $31.46_{-24.58}^{+64.44}$ & 202.82 & 89.27 & $1.5_{-0.5}^{+3.6}$ & II & -0.63 & -0.65 \\
\hline c2dJ033312.8+312124 & 3011659 & 3.8 & 8.95 & 0.28 & $1.38_{-1.23}^{+1.42}$ & $31.73_{-31.54}^{+40.29}$ & 92.66 & 507.28 & $1.0_{-0.0}^{+0.0}$ & $\mathrm{I}$ & 0.28 & 0.03 \\
\hline c2dJ033341.3+311341 & 3003019 & 2.6 & 2.59 & 0.23 & $1.88_{-1.33}^{+1.06}$ & $13.84_{-8.62}^{+76.16}$ & 888.1 & 12.61 & $1.7_{-0.7}^{+5.0}$ & II & -0.36 & -0.29 \\
\hline c2dJ034109.1+314438 & 3010092 & 3.5 & 5.51 & 0.26 & $2.15_{-1.35}^{+0.36}$ & $49.38_{-45.73}^{+47.77}$ & 1686.36 & 177.4 & $1.4_{-0.4}^{+2.1}$ & II & -0.51 & -0.53 \\
\hline c2dJ034255.9+315842 & 3019328 & 4.1 & 5.23 & 0.28 & $2.29_{-0.1}^{+0.02}$ & $65.84_{-2.06}^{+10.55}$ & 2437.85 & 62.43 & $5.3_{-4.3}^{+0.4}$ & III & -0.85 & -0.65 \\
\hline c2dJ034426.7+320820 & 3011061 & 0.6 & 0.84 & 0.3 & $0.21_{-0.06}^{+0.14}$ & $1.62_{-0.14}^{+2.62}$ & 37.06 & 17.83 & $1.1_{-0.1}^{+0.3}$ & I & -0.44 & 0.22 \\
\hline c2dJ034431.1+321848 & 3005792 & 1.2 & 3.05 & 0.26 & $0.13_{-0.03}^{+1.22}$ & $59.9_{-57.78}^{+38.47}$ & 3.09 & 2.97 & $3.7_{-2.7}^{+11.7}$ & II & -0.61 & -0.26 \\
\hline c2dJ034437.9+320804 & 3002764 & 0.9 & 3.92 & 0.26 & $0.8_{-0.53}^{+1.99}$ & $19.66_{-15.99}^{+54.92}$ & 93.56 & 37.84 & $1.3_{-0.3}^{+3.3}$ & II & -1.12 & -0.95 \\
\hline c2dJ034516.3+320620 & 3011667 & 2.6 & 5.22 & 0.23 & $1.08_{-0.28}^{+1.25}$ & $71.65_{-61.14}^{+19.15}$ & 56.7 & 49.01 & $1.4_{-0.4}^{+3.6}$ & II & -0.44 & -0.58 \\
\hline c2dJ034520.5+320634 & 3011715 & 0.4 & 4.48 & 0.28 & $1.53_{-1.11}^{+1.0}$ & $27.32_{-21.74}^{+66.14}$ & 266.2 & 26.14 & $1.2_{-0.2}^{+2.4}$ & II & -0.72 & -0.31 \\
\hline \multicolumn{13}{|c|}{$K_{s}$ band turnoff } \\
\hline c2dJ032741.5+302017 & 3001576 & 0.2 & 2.27 & 0.24 & $0.86_{-0.43}^{+0.97}$ & $31.32_{-21.27}^{+62.1}$ & 49.74 & 8.74 & $1.7_{-0.7}^{+3.6}$ & II & -0.67 & -0.24 \\
\hline c2dJ032800.1+300847 & 3000691 & 2.0 & 2.53 & 0.22 & $0.19_{-0.08}^{+0.26}$ & $5.3_{-3.45}^{+1 \overline{8} .02}$ & 25.08 & 7.42 & $1.2_{-0.2}^{+1.7}$ & II & -0.64 & -0.14 \\
\hline c2dJ032847.6+312406 & 3000851 & 0.2 & 8.46 & 0.23 & $0.18_{-0.07}^{+1.05}$ & $\begin{array}{l}12.99_{-10.93}^{+63.88} \\
-63\end{array}$ & 19.85 & 1.85 & $1.3_{-0.3}^{+2.6}$ & II & -1.07 & -0.58 \\
\hline c2dJ032850.6+304245 & 3014369 & 1.5 & 5.59 & 0.25 & $0.57_{-0.46}^{+1.21}$ & $11.45_{-9.57}^{+84.08}$ & 36.24 & 5.12 & $3.4_{-2.4}^{+31.8}$ & II & 0.04 & 0.16 \\
\hline c2dJ032851.0+311818 & 3017528 & 3.1 & 5.9 & 0.24 & $1.73_{-0.96}^{+0.37}$ & $45.46_{-38.29}^{+50.44}$ & 526.49 & 66.46 & $2.0_{-1.0}^{+5.4}$ & II & -0.65 & -0.34 \\
\hline c2dJ032851.2+311955 & 3013451 & 1.5 & 4.15 & 0.23 & $\begin{array}{l}1.11_{-0.61}^{+1.06} \\
\text { +..06 }\end{array}$ & $32.15_{-25.55}^{+61.02}$ & 169.12 & 18.34 & $10.8_{-9.8}^{+7.4}$ & III & -0.61 & -0.19 \\
\hline c2dJ032859.6+312147 & 3015522 & 3.6 & 2.25 & 0.2 & $2.24_{-0.88}^{+0.32}$ & $13.53_{-3.7}^{+10.05}$ & 904.71 & 12.41 & $1.6_{-0.6}^{+1.6}$ & II & -0.77 & -0.55 \\
\hline c2dJ032903.8+311604 & 3005745 & 2.3 & 4.9 & 0.25 & $0.82_{-0.65}^{+1.21}$ & $0.07_{-0.06}^{+0.78}$ & 1419.57 & 2497.43 & $3.7_{-2.7}^{+5.2}$ & I & 0.99 & 1.13 \\
\hline c2dJ032903.9+305630 & 3005573 & 1.7 & 0.65 & 0.25 & $0.14_{-0.04}^{+0.08}$ & $1.19_{-1.02}^{+5.31}$ & 14.67 & 5.3 & $13.6_{-12.6}^{+24.6}$ & I & 0.01 & 0.75 \\
\hline c2dJ032903.9+312149 & 3001003 & 1.4 & 3.29 & 0.23 & $0.99_{-0.55}^{+1.91}$ & $7.14_{-5.74}^{+69.25}$ & 206.45 & 25.3 & $2.0_{-1.0}^{+3.4}$ & II & -0.63 & -0.23 \\
\hline c2dJ032909.0+312624 & 3013017 & 2.0 & 2.11 & 0.22 & $0.59_{-0.48}^{+1.16}$ & $5.67_{-5.66}^{+79.27}$ & 102.75 & 0.72 & $2.2_{-1.2}^{+5.4}$ & II & 0.1 & 0.5 \\
\hline c2dJ032910.8+311643 & 3005025 & 2.7 & 1.25 & 0.24 & $0.3_{-0.19}^{+0.21}$ & $6.78_{-2.22}^{+3.66}$ & 11.28 & 2.45 & $5.2_{-4.2}^{+4.2}$ & $\mathrm{I}$ & -0.05 & 0.3 \\
\hline c2dJ032913.1+312253 & 3012963 & 0.2 & 2.57 & 0.22 & $0.27_{-0.15}^{+1.51}$ & $0.9_{-0.61}^{+3.96}$ & 90.31 & 53.94 & $1.2_{-0.2}^{+1.7}$ & I & -0.8 & -0.3 \\
\hline c2dJ032921.9+311536 & 3018372 & 6.0 & 0.77 & 0.22 & $0.2_{-0.07}^{+0.07}$ & $4.09_{-3.01}^{+11.73}$ & 34.96 & 14.09 & $1.2_{-0.2}^{+0.4}$ & II & -1.11 & -0.82 \\
\hline c2dJ032923.2+312030 & 3009928 & 0.3 & 0.89 & 0.24 & $0.13_{-0.03}^{+0.2}$ & $14.37_{-1}^{+}$ & 7.74 & 2.33 & $1.8_{-0.8}^{+8.9}$ & II & -0.69 & 0.03 \\
\hline c2dJ032932.6+312437 & 3003831 & 0.8 & 1.99 & 0.23 & $0.69_{-0.53}^{+1.36}$ & $13.76_{-7}^{+\frac{1}{5}}$ & 139.55 & 2.06 & $1.3_{-0.3}^{+0.2}$ & II & -0.91 & -0.38 \\
\hline c2dJ033001.9+303529 & 3010577 & 4.2 & 0.78 & 0.24 & $0.39_{-0.06}^{+0.13}$ & $8.17_{-0}^{+0 .}$ & 79.42 & 1.64 & $1.8_{-0.8}^{+1.3}$ & II & 0.82 & 1.06 \\
\hline c2dJ033035.9+303024 & 3009292 & 0.4 & 3.7 & 0.22 & $2.23_{-0.44}^{+0.67}$ & $39.41_{-29.54}^{+4.75}$ & 1083.2 & 125.66 & $1.4_{-0.4}^{+3.8}$ & II & -0.94 & -0.87 \\
\hline c2dJ033038.2+303212 & 3002146 & 1.6 & 0.9 & 0.24 & $0.35_{-0.13}^{+0.13}$ & $0.26_{-0.16}^{+1.62}$ & 179.08 & 170.26 & $55.6_{-54.6}^{+423.3}$ & I & 0.67 & 1.76 \\
\hline c2dJ033052.5+305418 & 3010241 & 0.6 & 6.53 & 0.23 & $0.57_{-0.46}^{+1.07}$ & $31.83_{-27.74}^{+0.16 .34}$ & 44.73 & 10.54 & $1.3_{-0.3}^{+13.2^{-5}}$ & II & -0.24 & -0.18 \\
\hline c2dJ033114.7+304955 & 3002775 & 2.4 & 2.89 & 0.25 & $2.41_{-1.06}^{+0.5}$ & $8.33_{-2.65}^{+3.41}$ & 1011.41 & 23.12 & $4.0_{-2.5}^{+3.6}$ & II & 0.04 & 0.01 \\
\hline c2dJ033142.4+310625 & 3016509 & 0.7 & 3.17 & 0.23 & $0.31_{-0.19}^{+0.28}$ & $\begin{array}{l}19.2_{-16.06}^{+37.9} \\
\end{array}$ & 37.18 & 3.41 & $1.3_{-0.3}^{+3.0}$ & II & -1.16 & -0.63 \\
\hline c2dJ033233.0+310222 & 3014845 & 3.6 & 3.21 & 0.22 & $0.2_{-0.09}^{+0.19}$ & $5.76_{-4.88}^{+13.31}$ & 68.83 & 16.19 & $\begin{array}{l}5.0_{-4.0}^{+1.9} \\
\end{array}$ & II & -0.89 & -0.58 \\
\hline c2dJ033234.0+310056 & 3000422 & 1.2 & 4.43 & 0.25 & $1.38_{-0.94}^{+1.08}$ & $22.39_{-14.91}^{+76.7}$ & 193.4 & 50.34 & $1.2_{-0.2}^{+2.0}$ & II & -0.46 & -0.28 \\
\hline
\end{tabular}

4 The YSOs start exhibiting significant ( $3 \sigma$ ) IR excesses above the photosphere level longward of the turnoff wavebands. 


\begin{tabular}{|c|c|c|c|c|c|c|c|c|c|c|c|c|}
\hline $\mathrm{ID}_{\mathrm{c} 2 \mathrm{~d}}$ & $\mathrm{ID}_{\text {model }}$ & $\overline{\chi_{r}^{2}}$ & $\overline{A_{V}}$ & $D$ & $M_{\star}$ & $\mathrm{Age}_{\star}$ & $L_{\star}$ & $L_{\text {dust }}$ & $R_{\text {in }} / R_{\text {sub }}$ & Stage & $\alpha_{\text {turnoff }}$ & $\alpha_{\text {excess }}$ \\
\hline (1) & (2) & (3) & $\begin{array}{l}(\mathrm{mag}) \\
(4)\end{array}$ & $\begin{array}{l}\text { (kpc) } \\
(5)\end{array}$ & $\begin{array}{l}\left(M_{\odot}\right) \\
(6)\end{array}$ & $\begin{array}{l}\left(10^{5} \mathrm{yr}\right) \\
(7)\end{array}$ & $\begin{array}{l}\left(10^{-2} L_{\odot}\right) \\
(8)\end{array}$ & $\begin{array}{l}\left(10^{-2} L_{\odot}\right) \\
(9)\end{array}$ & (10) & (11) & (12) & (13) \\
\hline c2dJ033241.7+311046 & 3010765 & 0.5 & 5.88 & 0.24 & $0.57_{-0.46}^{+0.18}$ & $64.2_{-61.8}^{+30.92}$ & 32.03 & 19.97 & $2.0_{-1.0}^{+13.6}$ & II & -0.29 & -0.01 \\
\hline c2dJ033401.7+311440 & 3009679 & 0.8 & 2.88 & 0.23 & $0.42_{-0.1}^{+0.43}$ & $9.33_{-4.2}^{+4.96}$ & 52.2 & 8.88 & $3.7_{-2.7}^{+4.0}$ & II & -0.92 & -0.7 \\
\hline c2dJ033915.8+312431 & 3007253 & 0.4 & 6.85 & 0.24 & $0.33_{-0.22}^{+1.03}$ & $35.06_{-32.25}^{+54.21}$ & 11.25 & 4.04 & $1.9_{-0.9}^{+6.3}$ & II & -0.71 & -0.24 \\
\hline c2dJ034119.2+320204 & 3002434 & 0.6 & 3.78 & 0.23 & $0.47_{-0.36}^{+0.92}$ & $43.27_{-37.0}^{+47.35}$ & 17.44 & 2.42 & $1.5_{-0.5}^{+3.4}$ & II & -1.19 & -0.89 \\
\hline c2dJ034155.7+314811 & 3008064 & 3.0 & 5.71 & 0.22 & $1.28_{-0.96}^{+1.13}$ & $6.5_{-0.81}^{+21.64}$ & 499.65 & 12.91 & $5.2_{-1.6}^{+1.1}$ & II & 0.27 & -0.03 \\
\hline c2dJ034157.8+314801 & 3007280 & 0.4 & 8.27 & 0.25 & $1.02_{-0.87}^{+0.75}$ & $22.39_{-21.01}^{+01}$ & 178.8 & 19.86 & $2.2_{-1.2}^{+1.8}$ & II & -0.71 & -0.36 \\
\hline c2dJ034219.3+314327 & 3017857 & 1.3 & 7.04 & 0.24 & $0.69_{-0.3}^{+1.64}$ & $37.15_{-30.81}^{+57.97}$ & 416.8 & 9.03 & $1.8_{-0.8}^{+4.1}$ & II & -0.4 & -0.25 \\
\hline c2dJ034232.9+314221 & 3016488 & 0.4 & 5.11 & 0.24 & $0.78_{-0.47}^{+0.93}$ & $51.63_{-41.79}^{+44.93}$ & 86.93 & 8.22 & $1.8_{-0.8}^{+6.0}$ & II & -0.66 & -0.17 \\
\hline c2dJ034322.2+314614 & 3005663 & 0.7 & 5.71 & 0.24 & $0.5_{-0.39}^{+1.33}$ & $24.68_{-22.92}^{+64.75}$ & 26.44 & 4.09 & $1.3_{-0.3}^{+3.0}$ & II & -0.95 & -0.53 \\
\hline c2dJ034328.2+320159 & 3018372 & 0.6 & 1.42 & 0.26 & $0.21_{-0.07}^{+0.33}$ & $7.2_{-5.58}^{+36.93}$ & 34.96 & 13.02 & $1.2_{-0.2}^{+1.5}$ & II & -1.21 & -0.91 \\
\hline c2dJ034355.2+315532 & 3002726 & 0.5 & 3.03 & 0.25 & $0.19_{-0.08}^{+1.29}$ & $2.52_{-1.52}^{+21.0}$ & 41.19 & 27.18 & $1.2_{-0.2}^{+1.4}$ & I & -0.56 & 0.32 \\
\hline c2dJ034356.0+320213 & 3005130 & 0.8 & 5.05 & 0.25 & $\begin{array}{l}0.71_{-0.48}^{+2.09} \\
\text { +2.09 }\end{array}$ & $4.63_{-3.46}^{+86.17}$ & 300.35 & 187.54 & $1.7_{-0.7}^{+3.2}$ & I & -0.82 & -0.49 \\
\hline c2dJ034358.6+321728 & 3010994 & 0.2 & 3.26 & 0.28 & $0.64_{-0.51}^{+0.82}$ & $33.03_{-29.73}^{+59.16}$ & 33.09 & 6.74 & $2.2_{-1.2}^{+7.8}$ & II & -0.83 & -0.27 \\
\hline c2dJ034358.9+321127 & 3004698 & 1.7 & 2.92 & 0.28 & $0.57_{-0.43}^{+1.71}$ & $21.17_{-19.34}^{+42.97}$ & 599.4 & 13.65 & $2.9_{-1.9}^{+4.0}$ & II & -0.43 & 0.28 \\
\hline c2dJ034359.9+320441 & 3012322 & 0.4 & 2.99 & 0.29 & $0.16_{-0.06}^{+0.18}$ & $67.5_{-50.52}^{+29.94}$ & 4.42 & 0.98 & $9.9_{-8.9}^{+24.0}$ & II & -0.95 & -0.03 \\
\hline c2dJ034406.0+321532 & 3013554 & 0.4 & 2.51 & 0.3 & $0.14_{-0.04}^{+0.15}$ & $75.1_{-53.34}^{+22.5}$ & 2.21 & 0.57 & $2.7_{-1.7}^{+10.8}$ & II & -1.06 & -0.5 \\
\hline c2dJ034406.8+320754 & 3014099 & 0.2 & 1.58 & 0.28 & $0.15_{-0.04}^{+0.24}$ & $27.98_{-24.21}^{+48.22}$ & 10.85 & 3.9 & $2.2_{-1.2}^{+8.5}$ & II & -0.97 & -0.32 \\
\hline c2dJ034407.5+320409 & 3006323 & 1.2 & 1.81 & 0.29 & $0.19_{-0.08}^{+0.26}$ & $5.75_{-5.45}^{+63.18}$ & 87.35 & 24.56 & $89.6_{-88.6}^{+33.3}$ & II & 0.1 & 1.3 \\
\hline c2dJ034411.6+320313 & 3017532 & 0.5 & 8.63 & 0.29 & $1.29_{-0.86}^{+0.86}$ & $23.94_{-17.17}^{+70.98}$ & 111.11 & 28.32 & $\begin{array}{l}1.5_{-0.5}^{+2.9} \\
\text { - }\end{array}$ & II & -0.95 & -0.63 \\
\hline c2dJ034418.6+321253 & 3005247 & 0.5 & 7.88 & 0.24 & $0.19_{-0.08}^{+1.65}$ & $8.93_{-8.07}^{+77.73}$ & 19.68 & 10.88 & $1.6_{-0.6}^{+4.6}$ & II & -0.84 & -0.78 \\
\hline c2dJ034421.6+321038 & 3015022 & 0.6 & 3.27 & 0.25 & $1.34_{-0.92}^{+1.55}$ & $9.54_{-6.48}^{+6.04}$ & 239.74 & 18.36 & $1.3_{-0.3}^{+2.0}$ & II & -1.0 & -0.72 \\
\hline c2dJ034422.3+321201 & 3016057 & 0.4 & 2.41 & 0.25 & $0.36_{-0.26}^{+0.26}$ & $37.51_{-31.02}^{+5.47}$ & 12.59 & 2.74 & $2.0_{-1.0}^{+5.0}$ & II & -1.01 & -0.63 \\
\hline c2dJ034425.5+321131 & 3014369 & 1.2 & 4.33 & 0.26 & $0.56_{-0.41}^{+1.6}$ & $31.08_{-27.33}^{+36.08}$ & 36.24 & 21.18 & $2.9_{-1.9}^{+7.6}$ & II & -0.53 & -0.24 \\
\hline c2dJ034427.3+321421 & 3010010 & 0.5 & 2.5 & 0.28 & $0.3_{-0.2}^{+0.67^{7}}$ & $36.19_{-32.14}^{+53.09}$ & 11.86 & 4.54 & $2.6_{-1.6}^{+10.4}$ & II & -0.96 & -0.28 \\
\hline c2dJ034431.4+320014 & 3007448 & 0.3 & 10.18 & 0.28 & $1.01_{-0.78}^{+0.81}$ & $22.73_{-19.04}^{+71.69}$ & 47.14 & 12.0 & $\begin{array}{l}1.9_{-0.9}^{+5.8} \\
\end{array}$ & II & -0.88 & -0.58 \\
\hline c2dJ034435.7+320304 & 3018693 & 0.6 & 2.74 & 0.26 & $0.31_{-0.21}^{+1.46}$ & $15.19_{-11.83}^{+72.83}$ & 194.13 & 4.48 & $1.7_{-0.7}^{+6.5}$ & II & -0.27 & 0.42 \\
\hline c2dJ034438.5+320736 & 3005663 & 3.5 & 2.35 & 0.23 & $0.45_{-0.29}^{+0.57}$ & $21.09_{-19.47}^{+45.4}$ & 26.44 & 9.05 & $1.8_{-0.8}^{+5.4}$ & II & -0.82 & -0.26 \\
\hline c2dJ034438.5+320801 & 3000691 & 0.7 & 2.01 & 0.26 & $0.21_{-0.1}^{+0.24}$ & $11.49_{-10.47}^{+16.18}$ & 25.08 & 8.14 & $8.0_{-7.0}^{+29.6}$ & II & -0.7 & 0.31 \\
\hline c2dJ034444.7+320402 & 3014306 & 0.4 & 4.54 & 0.29 & $1.73_{-1.31}^{+1.18}$ & $21.04_{-17.14}^{+72.38}$ & 845.66 & 21.02 & $1.3_{-0.3}^{+3.9}$ & II & -0.92 & -0.33 \\
\hline c2dJ034452.0+322625 & 3002305 & 0.6 & 3.48 & 0.23 & $0.26_{-0.15}^{+0.42}$ & $53.98_{-47}^{+42}$ & 8.36 & 0.85 & $1.3_{-0.3}^{+2.6}$ & II & -1.55 & -1.02 \\
\hline c2dJ034452.1+315825 & 3002882 & 0.1 & 4.97 & 0.28 & $0.27_{-0.17}^{+0.71}$ & $34.99_{-31}^{+57}$ & 35.96 & 2.84 & $1.4_{-0.4}^{+4.1}$ & II & -1.05 & -0.32 \\
\hline c2dJ034525.1+320930 & 3011159 & 1.4 & 2.34 & 0.24 & $0.23_{-0.12}^{+0.31}$ & $11.69_{-10.86}^{+19.06}$ & 37.64 & 16.06 & $5.1_{-4.1}^{+15.1}$ & II & -0.5 & 0.01 \\
\hline c2dJ034536.8+322557 & 3017240 & 0.4 & 3.33 & 0.24 & $1.49_{-0.86}^{+0.51}$ & $22.97_{-16.05}^{+53.94}$ & 160.5 & 25.0 & $1.3_{-0.3}^{+2.1}$ & II & -0.99 & -0.29 \\
\hline c2dJ034548.3+322412 & 3002927 & 1.9 & 0.38 & 0.24 & $2.69_{-1.05}^{+1.26}$ & $2.24_{-1.38}^{+1.33}$ & 2822.59 & 614.5 & $1.0_{-0.0}^{+0.0}$ & I & -0.72 & -0.11 \\
\hline c2dJ034558.2+322647 & 3000861 & 0.6 & 2.34 & 0.24 & $0.17_{-0.06}^{+0.18}$ & $53.97_{-44.72}^{+39.59}$ & 9.78 & 1.04 & $1.5_{-0.5}^{+3.0}$ & II & -1.33 & -0.8 \\
\hline \multicolumn{13}{|c|}{ IR1 band turnoff } \\
\hline c2dJ032747.7+301205 & 3015087 & 1.1 & 11.48 & 0.23 & $2.65_{-1.11}^{+0.73}$ & $33.44_{-23.92}^{+33.19}$ & 418.98 & 270.51 & $3.2_{-2.2}^{+4.6}$ & II & -0.21 & -0.52 \\
\hline c2dJ032834.5+310051 & 3000103 & 4.1 & 5.73 & 0.25 & $2.08_{-1.95}^{+1.66}$ & $3.88_{-3.84}^{+4.02}$ & 2102.0 & 79.64 & $5.7_{-4.7}^{+3.8}$ & II & 0.75 & 0.24 \\
\hline c2dJ032842.4+302953 & 3010255 & 0.3 & 0.85 & 0.23 & $0.16_{-0.05}^{+0.11}$ & $\begin{array}{l}6.72_{-4.96}^{+15.06} \\
\end{array}$ & 25.22 & 5.59 & $4.1_{-3.1}^{+7.0}$ & II & -0.74 & -0.37 \\
\hline c2dJ032844.1+312053 & 3015896 & 0.1 & 4.66 & 0.24 & $0.18_{-0.08}^{+0.2}$ & $64.38_{-44.49}^{+32.72}$ & 6.06 & 0.88 & $1.7_{-0.7}^{+5.5}$ & II & -1.04 & -0.54 \\
\hline c2dJ032846.2+311638 & 3005111 & 0.6 & 1.04 & 0.23 & $0.34_{-0.14}^{+0.24}$ & $12.12_{-6.91}^{+11.33}$ & 41.89 & 3.27 & $1.4_{-0.4}^{+2.7}$ & III & -1.31 & -0.81 \\
\hline c2dJ032847.8+311655 & 3017951 & 3.2 & 0.69 & 0.24 & $0.21_{-0.1}^{+0.15}$ & $3.47_{-2.98}^{+8.16}$ & 50.54 & 18.58 & $1.3_{-0.3}^{+9.8}$ & I & -0.77 & -0.37 \\
\hline c2dJ032852.2+312245 & 3016046 & 0.5 & 2.29 & 0.22 & $0.5_{-0.21}^{+0.37}$ & $39.73_{-21.76}^{+43.16}$ & 36.58 & 2.95 & $3.3_{-2.3}^{+9.6}$ & II & -0.83 & -0.45 \\
\hline c2dJ032856.6+311836 & 3019661 & 1.8 & 4.95 & 0.24 & $0.9_{-0.7}^{+1.8}$ & $6.41_{-5}^{+55}$ & 592.67 & 15.96 & $1.1_{-0.1}^{+0.9}$ & II & -0.3 & 0.19 \\
\hline c2dJ032857.0+311622 & 3000710 & 0.6 & 7.89 & 0.24 & $0.77_{-0 .}^{+0}$ & $29.8_{-27.81}^{+50.03}$ & 58.18 & 6.79 & $62.1_{-41.4}^{+43.1}$ & II & 0.43 & 0.97 \\
\hline c2dJ032903.1+312238 & 3008178 & 1.7 & 2.33 & 0.25 & $0.57_{-0.46}^{+1.59}$ & $11.51_{-10.46}^{+57.65}$ & 172.68 & 4.44 & $2.5_{-1.5}^{+8.8}$ & II & -0.21 & 0.26 \\
\hline c2dJ032904.1+305613 & 3008900 & 2.5 & 2.13 & 0.24 & $0.13_{-0.03}^{+0.09}$ & $71.02_{-43.79}^{+27.2}$ & 3.57 & 0.8 & $11.6_{-10.6}^{+17.5}$ & II & -0.37 & 0.29 \\
\hline c2dJ032918.7+312325 & 3013781 & 1.8 & 1.93 & 0.23 & $1.22_{-0.23}^{+1.0}$ & $29.04_{-22.44}^{+64.13}$ & 113.74 & 16.51 & $12.1_{-4.1}^{+6.1}$ & II & -0.08 & 0.09 \\
\hline c2dJ032920.4+311834 & 3010636 & 0.8 & 5.17 & 0.25 & $0.39_{-0.19}^{+0.96}$ & $1.33_{-1.09}^{+2.53}$ & 163.84 & 150.18 & $5.0_{-4.0}^{+13.9}$ & I & 0.03 & 0.15 \\
\hline c2dJ032930.4+311903 & 3006015 & 0.5 & 1.01 & 0.22 & $0.25_{-0.15}^{+0.33}$ & $34.79_{-30.14}^{+44.67}$ & 16.3 & 3.49 & $2.6_{-1.6}^{+6.3}$ & II & -0.63 & -0.22 \\
\hline c2dJ032932.9+312713 & 3010690 & 0.7 & 1.16 & 0.24 & $0.17_{-0.07}^{+0.15}$ & $63.83_{-28.44}^{+32.0}$ & 5.06 & 0.59 & $1.5_{-0.5}^{+4.0}$ & II & -1.16 & -0.6 \\
\hline c2dJ032937.7+312202 & 3008900 & 2.6 & 2.48 & 0.24 & $0.12_{-0.02}^{+0.14}$ & $73.95_{-70.37}^{+23.66}$ & 3.57 & 0.83 & $3.8_{-2.8}^{+38.0}$ & II & -0.56 & 0.18 \\
\hline
\end{tabular}




\begin{tabular}{|c|c|c|c|c|c|c|c|c|c|c|c|c|}
\hline $\mathrm{ID}_{\mathrm{c} 2 \mathrm{~d}}$ & $\mathrm{ID}_{\text {model }}$ & $\chi_{r}^{2}$ & $\overline{A_{V}}$ & $\bar{D}$ & $M_{\star}$ & $\mathrm{Age}_{\star}$ & $L_{\star}$ & $L_{\text {dust }}$ & $R_{\mathrm{in}} / R_{\mathrm{sub}}$ & Stage & $\alpha_{\text {turnoff }}$ & $\alpha_{\text {excess }}$ \\
\hline (1) & (2) & (3) & $\begin{array}{l}(\mathrm{mag}) \\
(4)\end{array}$ & $\begin{array}{l}\text { (kpc) } \\
(5)\end{array}$ & $\begin{array}{l}\left(M_{\odot}\right) \\
(6)\end{array}$ & $\begin{array}{l}\left(10^{5} \mathrm{yr}\right) \\
(7)\end{array}$ & $\begin{array}{l}\left(10^{-2} L_{\odot}\right) \\
(8)\end{array}$ & $\begin{array}{l}\left(10^{-2} L_{\odot}\right) \\
(9)\end{array}$ & (10) & (11) & (12) & (13) \\
\hline c2dJ032954.0+312053 & 3010241 & 1.2 & 3.17 & 0.22 & $0.99_{-0.44}^{+0.42}$ & $64.14_{-48.02}^{+29.03}$ & 44.73 & 9.72 & $2.6_{-1.6}^{+17.3}$ & II & -0.02 & 0.07 \\
\hline c2dJ033024.1+311404 & 3006161 & 1.3 & 0.91 & 0.25 & $0.12_{-0.02}^{+0.13}$ & $63.31_{-34.53}^{+34.13}$ & 6.61 & 0.64 & $5.0_{-4.0}^{+8.7}$ & II & -0.75 & -0.22 \\
\hline c2dJ033110.7+304941 & 3010687 & 1.9 & 3.45 & 0.25 & $0.85_{-0.38}^{+0.63}$ & $59.38_{-43.95}^{+30.1}$ & 40.35 & 5.43 & $4.5_{-3.5}^{+12.1}$ & II & -0.34 & -0.27 \\
\hline c2dJ033430.8+311324 & 3018409 & 0.2 & 2.7 & 0.23 & $0.14_{-0.04}^{+0.26}$ & $26.83_{-22.89}^{+35.33}$ & 7.67 & 1.1 & $2.8_{-1.8}^{+7.7}$ & II & -0.94 & -0.57 \\
\hline c2dJ033449.8+311550 & 3016746 & 0.3 & 1.93 & 0.23 & $0.83_{-0.4}^{+0.71}$ & $25.84_{-16.14}^{+58.6}$ & 48.62 & 4.7 & $1.2_{-0.2}^{+1.3}$ & II & -0.91 & -0.42 \\
\hline c2dJ034001.5+311017 & 3016746 & 0.2 & 5.0 & 0.24 & $0.35_{-0.23}^{+0.63}$ & $59.12_{-49.83}^{+39.29}$ & 48.62 & 1.07 & $1.3_{-0.3}^{+3.5}$ & II & -1.28 & -0.76 \\
\hline c2dJ034201.0+314913 & 3003178 & 0.1 & 6.19 & 0.24 & $0.2_{-0.1}^{+0.28}$ & $58.81_{-43.0}^{+36.99}$ & 7.31 & 1.03 & $1.6_{-0.6}^{+5.0}$ & II & -1.12 & -0.6 \\
\hline c2dJ034204.3+314712 & 3013699 & 0.1 & 5.51 & 0.23 & $0.14_{-0.04}^{+0.46}$ & $19.45_{-16.18}^{+57.42}$ & 15.15 & 3.18 & $1.1_{-0.1}^{+9.7}$ & II & -0.71 & -0.44 \\
\hline c2dJ034220.3+320531 & 3017175 & 0.2 & 3.5 & 0.25 & $0.51_{-0.25}^{+0.32}$ & $67.5_{-36.31}^{+29.65}$ & 20.97 & 2.75 & $1.2_{-0.2}^{+17.7}$ & II & -0.68 & -0.17 \\
\hline c2dJ034232.1+315250 & 3011391 & 0.5 & 3.92 & 0.25 & $0.17_{-0.07}^{+0.26}$ & $73.52_{-52.8}^{+22.22}$ & 1.88 & 0.28 & $2.6_{-1.6}^{+16.7}$ & II & -1.07 & -0.5 \\
\hline c2dJ034249.2+315011 & 3014019 & 0.3 & 4.77 & 0.23 & $0.25_{-0.14}^{+0.59}$ & $24.7_{-21.55}^{+58.42}$ & 19.62 & 2.9 & $1.3_{-0.3}^{+3.8}$ & II & -1.2 & -0.74 \\
\hline c2dJ034313.7+320045 & 3009840 & 1.2 & 7.33 & 0.24 & $0.37_{-0.26}^{+0.71}$ & $51.38_{-46.53}^{+33.56}$ & 52.12 & 1.37 & $8.5_{-7.5}^{+9.5}$ & II & -0.13 & 0.08 \\
\hline c2dJ034323.6+321226 & 3010066 & 0.4 & 2.54 & 0.29 & $0.18_{-0.08}^{+0.22}$ & $61.75_{-42.78}^{+35.72}$ & 5.67 & 0.71 & $1.4_{-0.4}^{+4.4}$ & II & -1.26 & -0.59 \\
\hline c2dJ034329.4+315219 & 3019301 & 1.9 & 3.34 & 0.23 & $0.23_{-0.05}^{+0.66}$ & $1.41_{-1.01}^{+5.52}$ & 351.25 & 19.7 & $20.6_{-3.8}^{+9.7}$ & II & 0.95 & 0.96 \\
\hline c2dJ034345.2+320359 & 3018441 & 0.8 & 4.83 & 0.24 & $0.23_{-0.12}^{+1.2}$ & $0.2_{-0.18}^{+3.35}$ & 228.28 & 130.68 & $7.9_{-6.9}^{+25.0}$ & I & 0.65 & 0.54 \\
\hline c2dJ034348.8+321552 & 3003975 & 0.3 & 1.81 & 0.26 & $0.17_{-0.07}^{+0.37}$ & $32.9_{-28.96}^{+52.01}$ & 8.42 & 1.38 & $5.4_{-4.4}^{+9.8}$ & II & -0.71 & -0.4 \\
\hline c2dJ034355.3+320753 & 3013176 & 0.3 & 2.46 & 0.28 & $0.15_{-0.05}^{+0.2}$ & $50.98_{-38.41}^{+46.11}$ & 8.72 & 1.08 & $3.2_{-2.2}^{+10.3}$ & II & -0.91 & -0.41 \\
\hline c2dJ034359.1+321421 & 3015397 & 1.6 & 3.03 & 0.29 & $1.3_{-0.71}^{+0.57}$ & $\begin{array}{l}21.07_{-15.06}^{+61.01} \\
+61\end{array}$ & 243.39 & 8.35 & $1.2_{-0.2}^{+3.2}$ & II & -0.37 & 0.03 \\
\hline c2dJ034401.6+322359 & 3002465 & 0.3 & 2.42 & 0.24 & $0.18_{-0.08}^{+0.21}$ & $66.82_{-37.32}^{+29.88}$ & 3.66 & 0.68 & $4.2_{-3.2}^{+16.9}$ & II & -0.84 & -0.24 \\
\hline c2dJ034402.9+315228 & 3007428 & 0.0 & 5.23 & 0.25 & $0.18_{-0.07}^{+0.27}$ & $70.73_{-56.06}^{+26.74}$ & 3.53 & 0.42 & $1.9_{-0.9}^{+9.3}$ & II & -1.06 & -0.6 \\
\hline c2dJ034418.2+320457 & 3006383 & 1.4 & 1.87 & 0.3 & $0.39_{-0.22}^{+2.47}$ & $2.04_{-1.55}^{+52.9}$ & 162.9 & 113.41 & $9.2_{-8.2}^{+94.7}$ & I & 0.14 & 1.08 \\
\hline c2dJ034425.5+320617 & 3007450 & 0.3 & 5.49 & 0.28 & $0.72_{-0.39}^{+0.53}$ & $51.71_{-34.51}^{+42.9}$ & 100.38 & 1.99 & $9.6_{-8.6}^{+3.2 .9}$ & II & -0.43 & -0.05 \\
\hline c2dJ034426.0+320430 & 3014489 & 1.2 & 2.79 & 0.28 & $\begin{array}{l}1.09_{-0.48}^{+2.38} \\
\end{array}$ & $4.67_{-4.03}^{+70.23}$ & 1402.83 & 224.54 & $1.7_{-0.7}^{+4.4}$ & I & -0.65 & -0.01 \\
\hline c2dJ034427.9+322719 & 3011356 & 1.5 & 2.8 & 0.23 & $0.18_{-0.08}^{+0.15}$ & $19.7_{-17.94}^{+18.91}$ & 34.0 & 3.89 & $2.1_{-1.1}^{+5.4}$ & II & -0.78 & -0.22 \\
\hline c2dJ034428.5+315954 & 3003640 & 0.2 & 1.36 & 0.25 & $0.22_{-0.11}^{+0.27}$ & $19.28_{-16.15}^{+27.87}$ & 15.25 & 1.74 & $1.5_{-0.5}^{+5.4}$ & II & -1.1 & -0.47 \\
\hline c2dJ034429.8+320055 & 3011338 & 0.4 & 3.58 & 0.29 & $0.16_{-0.06}^{+0.32}$ & $38.8_{-33.65}^{+51.83}$ & 10.85 & 1.7 & $3.0_{-2.0}^{+8.8}$ & II & -0.73 & -0.27 \\
\hline c2dJ034432.0+321144 & 3016117 & 1.3 & 6.25 & 0.33 & $2.42_{-1.78}^{+1.25}$ & $33.81_{-29.25}^{+57.04}$ & 7593.6 & 106.15 & $21.7_{-14.7}^{+23.3}$ & III & -0.03 & -0.17 \\
\hline c2dJ034433.8+315830 & 3006115 & 0.5 & 4.07 & 0.28 & $0.36_{-0.26}^{+0.48}$ & $47.97_{-43.92}^{+42.66}$ & 24.01 & 5.23 & $2.9_{-1.9}^{+26.4}$ & II & -0.59 & -0.02 \\
\hline c2dJ034435.0+321531 & 3015526 & 0.3 & 2.46 & 0.26 & $0.18_{-0.07}^{+0.34}$ & $36.6_{-31.73}^{+53.57}$ & 9.91 & 1.01 & $\begin{array}{l}3.0_{-2.0}^{+7.9} \\
\end{array}$ & II & -0.52 & -0.2 \\
\hline c2dJ034435.5+320856 & 3013517 & 1.6 & 1.92 & 0.29 & $0.2_{-0.09}^{+0.39}$ & $0.31_{-0.29}^{+2.09}$ & 119.01 & 246.57 & $72.9_{-71.9}^{+66.6}$ & I & 1.37 & 2.43 \\
\hline c2dJ034437.0+320645 & 3002420 & 0.3 & 2.34 & 0.22 & $1.82_{-1.29}^{+0.94}$ & $14.61_{-10.0}^{+61.69}$ & 1130.45 & 35.64 & $1.7_{-0.7}^{+2.4}$ & III & -1.45 & -0.82 \\
\hline c2dJ034437.4+320901 & 3002879 & 2.5 & 2.08 & 0.26 & $0.74_{-0.45}^{+1.43}$ & $2.62_{-2.12}^{+45.67}$ & 566.14 & 131.15 & $57.9_{-56.9}^{+55.4}$ & I & 0.47 & 0.77 \\
\hline c2dJ034438.0+320330 & 3008445 & 1.5 & 3.98 & 0.3 & $1.23_{-0.74}^{+0.57}$ & $28.25_{-20.24}^{+66.3}$ & 215.36 & 17.42 & $8.0_{-7.0}^{+10.1}$ & II & -0.19 & 0.16 \\
\hline c2dJ034439.8+321804 & 3003226 & 0.3 & 3.51 & 0.24 & $0.49_{-0.33}^{+0.67}$ & $32.89_{-19.7}^{+56.74}$ & 22.26 & 2.42 & $1.7_{-0.7}^{+8.1}$ & II & -0.82 & -0.24 \\
\hline c2dJ034440.2+320933 & 3019351 & 1.7 & 1.23 & 0.23 & $0.47_{-0.35}^{+0.65}$ & $0.47_{-0.41}^{+0.95}$ & 516.47 & 412.3 & $101.0_{-100.0}^{+54.7}$ & I & 1.53 & 1.73 \\
\hline c2dJ034442.6+321002 & 3005022 & 1.3 & 3.16 & 0.26 & $0.18_{-0.08}^{+1.39}$ & $1.31_{-1.01}^{+83.53}$ & 691.28 & 133.77 & $52.8_{-51.8}^{+55.8}$ & I & 0.57 & 1.42 \\
\hline c2dJ034443.1+313734 & 3016867 & 0.2 & 6.22 & 0.24 & $0.24_{-0.13}^{+0.25}$ & $65.64_{-44.68}^{+31.04}$ & 5.8 & 0.5 & $1.1_{-0.1}^{+36.1}$ & II & -0.75 & -0.28 \\
\hline c2dJ034443.8+321030 & 3014727 & 1.1 & 3.24 & 0.25 & $0.16_{-0.05}^{+0.1}$ & $6.24_{-4.98}^{+11.78^{\circ}}$ & 45.58 & 21.73 & $13.3_{-8.2}^{+14.1}$ & I & -0.04 & 0.44 \\
\hline c2dJ034450.4+315236 & 3007414 & 0.2 & 3.46 & 0.25 & $0.16_{-0.06}^{+0.33}$ & $77.89_{-72.68}^{+19.55}$ & 3.63 & 0.71 & $2.9_{-1.9}^{+13.4}$ & II & -0.82 & -0.18 \\
\hline c2dJ034456.1+320915 & 3011184 & 1.4 & 3.73 & 0.28 & $1.4_{-0.78}^{+1.24}$ & $26.49_{-21}^{+65}$ & 945.42 & 46.81 & $3.2_{-2.2}^{+37.1}$ & II & -0.27 & 0.53 \\
\hline c2dJ034517.8+321206 & 3003343 & 0.5 & 2.31 & 0.28 & $0.18_{-0.08}^{+0.24}$ & $53.74_{-42.93}^{+42.09}$ & 9.66 & 1.24 & $1.6_{-0.6}^{+5.2}$ & II & -1.24 & -0.55 \\
\hline c2dJ034529.7+315920 & 3012232 & 0.2 & 4.46 & 0.25 & $0.18_{-0.07}^{+0.18}$ & $79.08_{-35.38}^{+18.53}$ & 3.63 & 0.54 & $5.6_{-4.6}^{+21.3}$ & II & -0.82 & -0.25 \\
\hline c2dJ034533.5+314555 & 3010281 & 0.1 & 5.97 & 0.25 & $0.16_{-0.06}^{+0.3}$ & $79.23_{-56.63}^{+18.30}$ & 3.49 & 0.41 & $3.4_{-2.4}^{+17.2}$ & II & -0.84 & -0.34 \\
\hline c2dJ034535.6+315954 & 3018513 & 0.1 & 5.1 & 0.24 & $0.24_{-0.13}^{+0.39}$ & $44.58_{-39.23}^{+48.99}$ & 13.93 & 1.08 & $4.4_{-3.4}^{+16.0}$ & II & -0.73 & -0.22 \\
\hline c2dJ034657.4+324917 & 3000062 & 0.1 & 2.52 & 0.24 & $0.19_{-0.08}^{+0.22}$ & $64.34_{-42.38}^{+32.75}$ & 5.7 & 1.0 & $1.5_{-0.5}^{+4.3}$ & II & -1.14 & -0.47 \\
\hline \multicolumn{13}{|c|}{ IR2 band turnoff } \\
\hline c2dJ032851.1+311632 & 3009474 & 0.7 & 1.75 & 0.24 & $0.16_{-0.06}^{+0.15}$ & $54.6_{-31.98}^{+36.54}$ & 7.84 & 0.5 & $1.5_{-0.5}^{+4.1}$ & II & -1.16 & -0.76 \\
\hline c2dJ032852.2+311547 & 3013176 & 0.8 & 2.88 & 0.24 & $0.14_{-0.03}^{+0.11}$ & $37.52_{-3}^{+2}$ & 8.72 & 0.84 & $2.1_{-1.1}^{+7.5}$ & II & -1.04 & -0.82 \\
\hline c2dJ032852.9+311626 & 3019740 & 0.7 & 1.81 & 0.25 & $0.17_{-0.06}^{+0.15}$ & $70.91_{-33.59}^{+26.7}$ & 3.79 & 0.38 & $1.5_{-0.5}^{+4.8}$ & II & -0.43 & 0.22 \\
\hline c2dJ032909.5+312721 & 3006161 & 0.1 & 4.07 & 0.24 & $0.19_{-0.09}^{+0.21}$ & $72.04_{-46.23}^{+25.06}$ & 6.61 & 0.84 & $10.9_{-9.9}^{+10.2}$ & II & -0.54 & -0.34 \\
\hline c2dJ032917.8+311948 & 3014115 & 0.1 & 5.88 & 0.25 & $0.18_{-0.07}^{+0.19}$ & $72.46_{-48.93}^{+25.01}$ & 5.91 & 0.76 & $2.8_{-1.8}^{+13.0}$ & II & -0.71 & -0.38 \\
\hline c2dJ032921.6+312110 & 3018513 & 1.0 & 1.81 & 0.23 & $0.3_{-0.19}^{+0.26}$ & $43.7_{-30.16}^{+48.98}$ & 13.93 & 0.99 & $3.4_{-2.4}^{+15.4}$ & II & -0.76 & -0.25 \\
\hline
\end{tabular}




\begin{tabular}{|c|c|c|c|c|c|c|c|c|c|c|c|c|}
\hline $\mathrm{ID}_{\mathrm{C} 2 \mathrm{~d}}$ & $\mathrm{ID}_{\text {model }}$ & $\chi_{r}^{2}$ & $A_{V}$ & $D$ & $M_{\star}$ & $\mathrm{Age}_{\star}$ & $L_{\star}$ & $L_{\text {dust }}$ & $R_{\mathrm{in}} / R_{\mathrm{sub}}$ & Stage & $\alpha_{\text {turnoff }}$ & $\alpha_{\text {excess }}$ \\
\hline (1) & (2) & (3) & $\begin{array}{l}\text { (mag) } \\
\text { (4) }\end{array}$ & $\begin{array}{l}(\mathrm{kpc}) \\
(5)\end{array}$ & $\begin{array}{l}\left(M_{\odot}\right) \\
(6)\end{array}$ & $\begin{array}{l}\left(10^{5} \mathrm{yr}\right) \\
(7)\end{array}$ & $\begin{array}{l}\left(10^{-2} L_{\odot}\right) \\
(8)\end{array}$ & $\begin{array}{l}\left(10^{-2} L_{\odot}\right) \\
(9)\end{array}$ & (10) & (11) & (12) & (13) \\
\hline $\mathrm{c} 2 \mathrm{dJ} 032923.2+312653$ & 3000123 & 0.6 & 2.21 & 0.24 & $0.14_{-0.04}^{+0.18}$ & $41.76_{-37.62}^{+38.53}$ & 34.54 & 0.8 & $2.2_{-1.2}^{+8.4}$ & II & -0.26 & 0.22 \\
\hline c2dJ032928.9+305842 & 3008430 & 1.3 & 0.62 & 0.25 & $0.13_{-0.03}^{+0.21}$ & $48.8_{-42.55}^{+35.62}$ & 4.97 & 0.66 & $19.1_{-8.2}^{+11.7}$ & II & -0.26 & 0.14 \\
\hline c2dJ032929.8+312103 & 3005493 & 0.3 & 1.91 & 0.23 & $0.14_{-0.04}^{+0.23}$ & $16.81_{-13.88}^{+34.57}$ & 22.63 & 2.38 & $9.2_{-8.2}^{+14.9}$ & II & -0.45 & -0.16 \\
\hline c2dJ032937.6+310249 & 3008677 & 0.2 & 1.69 & 0.26 & $0.1_{-0.0}^{+0.17}$ & $91.2_{-32.9}^{+3.96}$ & 1.5 & 0.14 & $8.3_{-7.3}^{+17.8}$ & II & -0.77 & -0.26 \\
\hline c2dJ033120.1+304918 & 3000664 & 0.4 & 3.05 & 0.25 & $0.12_{-0.02}^{+0.22}$ & $35.59_{-34.47}^{+42.75}$ & 52.32 & 4.94 & $4.1_{-3.1}^{+12.3}$ & I & -0.21 & 0.28 \\
\hline c2dJ033346.9+305350 & 3014115 & 0.5 & 1.83 & 0.25 & $0.16_{-0.06}^{+0.13}$ & $71.56_{-32.6}^{+26.04}$ & 5.91 & 0.72 & $2.8_{-1.8}^{+13.1}$ & II & -0.59 & -0.01 \\
\hline c2dJ034336.0+315009 & 3004647 & 1.3 & 0.7 & 0.22 & $0.3_{-0.07}^{+0.65}$ & $14.73_{-7.96}^{+41.19}$ & 19.78 & 1.32 & $16.8_{-15.8}^{+108.3}$ & II & 0.2 & 0.66 \\
\hline c2dJ034346.5+321106 & 3018345 & 0.2 & 3.4 & 0.33 & $0.14_{-0.04}^{+0.2}$ & $83.29_{-55.22}^{+15.22}$ & 2.63 & 0.19 & $3.2_{-2.2}^{+17.9}$ & II & 0.15 & 0.72 \\
\hline c2dJ034347.6+320903 & 3008677 & 0.4 & 3.21 & 0.32 & $0.13_{-0.03}^{+0.12}$ & $83.55_{-34.86}^{+14.67}$ & 1.5 & 0.16 & $5.3_{-4.3}^{+15.7}$ & II & -0.59 & -0.24 \\
\hline c2dJ034415.8+315937 & 3019728 & 0.2 & 2.9 & 0.28 & $0.17_{-0.07}^{+0.17}$ & $67.5_{-50.52}^{+30.00}$ & 4.18 & 0.49 & $\begin{array}{r}11.2_{-10.2}^{+17.9} \\
\text { +17. }\end{array}$ & II & -0.4 & 0.04 \\
\hline c2dJ034421.3+321156 & 3004577 & 0.5 & 2.71 & 0.26 & $0.48_{-0.26}^{+0.41}$ & $54.12_{-31.05}^{+40.45}$ & 14.56 & 1.23 & $4.5_{-3.5}^{+11.9}$ & II & 0.01 & 0.14 \\
\hline c2dJ034427.2+322029 & 3019764 & 0.3 & 2.28 & 0.29 & $0.18_{-0.08}^{+0.24}$ & $64.37_{-40.1}^{+33.07}$ & 7.72 & 0.68 & $3.5_{-2.5}^{+17.5}$ & II & -0.82 & -0.27 \\
\hline c2dJ034430.8+320956 & 3020088 & 1.1 & 2.38 & 0.33 & $2.16_{-0.33}^{+0.49}$ & $60.88_{-18.89}^{+15.57}$ & 4537.84 & 18.11 & $41.0_{-24.4}^{+20.6}$ & III & 0.02 & 0.01 \\
\hline c2dJ034658.5+324659 & 3002972 & 0.4 & 3.17 & 0.24 & $0.21_{-0.1}^{+0.22}$ & $56.68_{-39.94}^{+39.12}$ & 5.56 & 0.52 & $1.7_{-0.7}^{+5.4}$ & II & -1.16 & -0.65 \\
\hline \multicolumn{13}{|c|}{ IR3 band turnoff } \\
\hline c2dJ032858.1+311804 & 3011544 & 0.3 & 2.56 & 0.25 & $0.48_{-0.17}^{+0.26}$ & $69.2_{-35.51}^{+28.95}$ & 19.21 & 0.2 & $55.8_{-54.8}^{+886.5}$ & III & -1.97 & -1.38 \\
\hline c2dJ032908.0+312251 & 3019657 & 1.0 & 3.08 & 0.23 & $0.44_{-0.3}^{+1.21}$ & $0.7_{-0.65}^{+3.88}$ & 625.91 & 216.22 & $52.4_{-33.3}^{+40.5}$ & I & 1.52 & 1.86 \\
\hline c2dJ032912.9+312329 & 3011599 & 1.9 & 0.48 & 0.23 & $0.19_{-0.06}^{+0.12}$ & $0.81_{-0.14}^{+1.59}$ & 55.98 & 26.53 & $72.1_{-9.4}^{+16.5}$ & I & 1.6 & 2.17 \\
\hline c2dJ032916.8+312325 & 3000622 & 2.3 & 2.16 & 0.23 & $0.78_{-0.62}^{+1.53}$ & $0.15_{-0.13}^{+96.33}$ & 33.61 & 2.46 & $11.4_{-10.4}^{+32.8}$ & II & 0.6 & 1.04 \\
\hline c2dJ032926.8+312648 & 3015385 & 3.5 & 1.64 & 0.22 & $0.59_{-0.11}^{+0.84}$ & $18.13_{-3.32}^{+61.17}$ & 75.58 & 0.75 & $615.6_{-422.5}^{+1618.4}$ & II & -1.06 & -0.02 \\
\hline c2dJ032929.3+311835 & 3015686 & 1.7 & 3.48 & 0.25 & $0.53_{-0.07}^{+0.51}$ & $30.84_{-10.9}^{+68.48}$ & 35.7 & 7.53 & $213.6_{-122.1}^{+129.2}$ & II & 1.16 & 1.26 \\
\hline c2dJ034233.1+315215 & 3014024 & 0.2 & 4.67 & 0.24 & $0.11_{-0.01}^{+0.2}$ & $71.51_{-45.7}^{+25.59}$ & 14.75 & 0.36 & $21.0_{-20.0}^{+32.7}$ & II & -0.02 & 0.45 \\
\hline c2dJ034234.2+315101 & 3003543 & 0.1 & 3.79 & 0.23 & $0.12_{-0.01}^{+0.14}$ & $79.74_{-38.41}^{+20.05}$ & 3.43 & 0.24 & $55.7_{-30.8}^{+52.5}$ & II & 0.27 & 0.85 \\
\hline c2dJ034250.9+314045 & 3005351 & 0.4 & 5.92 & 0.24 & $0.27_{-0.17}^{+0.41}$ & $51.31_{-43.19}^{+46.16}$ & 13.01 & 0.22 & $44.8_{-43.8}^{+265.4}$ & II & -1.14 & 0.47 \\
\hline c2dJ034301.9+314436 & 3016352 & 2.7 & 4.22 & 0.25 & $0.13_{-0.01}^{+0.19}$ & $11.99_{-7.77}^{+14.19}$ & 10.12 & 1.14 & $28.8_{-27.7}^{+93.3}$ & I & 0.75 & 1.55 \\
\hline c2dJ034308.7+315139 & 3002609 & 1.8 & 3.71 & 0.22 & $0.28_{-0.17}^{+0.09}$ & $2.65_{-2.1}^{+6.54}$ & 11.87 & 1.18 & $72.4_{-24.1}^{+69.0}$ & II & 1.53 & 2.11 \\
\hline c2dJ034344.6+320818 & 3018287 & 0.8 & 2.42 & 0.25 & $0.82_{-0.32}^{+0.96}$ & $\begin{array}{l}52.04_{-49.88}^{+27.79} \\
\end{array}$ & 72.61 & 2.56 & $79.9_{-78.2}^{+297.5}$ & II & 0.85 & 1.75 \\
\hline c2dJ034410.1+320405 & 3018669 & 1.5 & 1.5 & 0.32 & $0.16_{-0.06}^{+0.29}$ & $0.1_{-0.07}^{+1.21}$ & 76.01 & 118.91 & $73.8_{-72.0}^{+65.8}$ & I & 1.5 & 1.63 \\
\hline c2dJ034415.2+321942 & 3015752 & 1.4 & 3.47 & 0.31 & $0.2_{-0.09}^{+0.67}$ & $40.65_{-17.14}^{+30.57}$ & 8.3 & 1.98 & $43.2_{-17.7}^{+98.3}$ & II & 0.81 & 1.23 \\
\hline c2dJ034418.2+320959 & 3005606 & 0.5 & 3.73 & 0.26 & $0.24_{-0.13}^{+0.25}$ & $34.14_{-29.1}^{+58.68}$ & 19.59 & 1.02 & $14.3_{-13.3}^{+49.7}$ & II & -0.54 & 0.09 \\
\hline c2dJ034422.3+320543 & 3009294 & 0.7 & 2.09 & 0.21 & $1.0_{-0.51}^{+1.01}$ & $10.57_{-1.17}^{+16.26}$ & 162.02 & 5.49 & $41.5_{-17.5}^{+17.8}$ & II & 0.46 & 0.54 \\
\hline c2dJ034422.6+320154 & 3004647 & 0.7 & 1.96 & 0.25 & $0.25_{-0.14}^{+0.37}$ & $20.3_{-18.27}^{+4.1}$ & 19.78 & 1.92 & $99.5_{-55.7}^{+84.5}$ & II & 0.87 & 1.87 \\
\hline c2dJ034425.7+321549 & 3009409 & 2.0 & 1.1 & 0.28 & $0.15_{-0.04}^{+0.59}$ & $0.93_{-0.9}^{+66.26}$ & 33.92 & 19.34 & $65.7_{-64.7}^{+75.7}$ & I & 1.71 & 2.21 \\
\hline c2dJ034429.2+320116 & 3002365 & 0.4 & 7.04 & 0.28 & $0.34_{-0.24}^{+0.54}$ & $44.01_{-38.97}^{+49.91}$ & 16.92 & 0.4 & $\begin{array}{l}44.4_{-43.4}^{+61.9} \\
\text { +61. }\end{array}$ & II & 0.2 & 1.04 \\
\hline c2dJ034429.7+321040 & 3002351 & 1.5 & 1.42 & 0.29 & $\begin{array}{l}0.59_{-0.08}^{+0.98} \\
\end{array}$ & $5.42_{-0.4}^{+7.40 .91}$ & 198.15 & 11.22 & $27.1_{-4.3}^{+32.2}$ & II & 0.5 & 0.76 \\
\hline c2dJ034434.1+321636 & 3014280 & 0.1 & 3.27 & 0.28 & $0.19_{-0.09}^{+0.4}$ & $63.79_{-52.24}^{+33.36}$ & 16.02 & 0.38 & $3.4_{-2.4}^{+11.3}$ & II & 0.09 & 0.63 \\
\hline c2dJ034434.8+315655 & 3007580 & 1.5 & 2.26 & 0.25 & $0.29_{-0.19}^{+0.29}$ & $42.37_{-37.53}^{+42.76}$ & 19.76 & 0.61 & $1.3_{-0.3}^{+86.6}$ & II & 0.11 & 1.26 \\
\hline c2dJ034437.4+321224 & 3000393 & 0.5 & 6.22 & 0.3 & $0.92_{-0.7}^{+0.57}$ & $42.8_{-31.37}^{+37.03}$ & 189.15 & 5.21 & $52.9_{-26.2}^{+4.32 .3}$ & II & 0.56 & 0.93 \\
\hline c2dJ034438.0+321137 & 3003770 & 0.8 & 0.9 & 0.25 & $0.21_{-0.09}^{+0.06}$ & $4.94_{-3.54}^{+36.18}$ & 40.14 & 4.68 & $46.0_{-22.9}^{+32.6}$ & II & 0.91 & 1.7 \\
\hline c2dJ034439.0+320320 & 3015863 & 4.2 & 1.27 & 0.32 & $0.13_{-0.03}^{+0.15}$ & $0.05_{-0.02}^{+1.24}$ & 64.66 & 31.03 & $57.3_{-4.7}^{+49.0}$ & I & 2.15 & 2.06 \\
\hline c2dJ034439.2+322009 & 3003660 & 1.5 & 2.25 & 0.3 & $0.13_{-0.03}^{+0.09}$ & $5.46_{-4.45}^{+13.76}$ & 18.89 & 8.56 & $61.9_{-22.7}^{+61.0}$ & II & 1.23 & 1.71 \\
\hline c2dJ034441.7+321202 & 3004538 & 1.4 & 4.54 & 0.26 & $0.32_{-0.21}^{+0.92}$ & $29.6_{-27.56}^{+59.14}$ & 26.64 & 14.13 & $94.0_{-68.4}^{+114.1}$ & II & 0.27 & 1.32 \\
\hline c2dJ034442.6+320619 & 3000760 & 1.2 & 1.1 & 0.32 & $0.63_{-0.17}^{+0.62}$ & $68.52_{-67.99}^{+20.32}$ & 24.63 & 5.15 & $79.0_{-35.6}^{+137.0}$ & II & 1.39 & 2.32 \\
\hline c2dJ034443.0+321560 & 3010384 & 1.1 & 1.9 & 0.29 & $0.19_{-0.08}^{+0.06}$ & $1.17_{-0.67}^{+1.22}$ & 68.55 & 23.57 & $38.5_{-0.9}^{+50.2}$ & I & 0.71 & 0.7 \\
\hline c2dJ034444.6+320813 & 3006152 & 1.4 & 0.76 & 0.32 & $0.2_{-0.1}^{+0.23}$ & $0.5_{-0.48}^{+2.69}$ & 199.18 & 127.76 & $32.3_{-31.3}^{+39.8}$ & I & 1.24 & 1.85 \\
\hline c2dJ034457.9+320402 & 3010073 & 0.6 & 1.86 & 0.28 & $0.16_{-0.06}^{+0.15}$ & $50.83_{-41.65}^{+40.66}$ & 7.9 & 0.29 & $2.4_{-1.4}^{+16.0}$ & II & -1.35 & -1.07 \\
\hline c2dJ034460.0+322233 & 3018345 & 0.1 & 2.53 & 0.25 & $0.13_{-0.03}^{+0.21}$ & $87.96_{-39.16}^{+10.55}$ & 2.63 & 0.2 & $7.9_{-6.9}^{+21.2}$ & II & -0.34 & 0.04 \\
\hline c2dJ034501.4+320502 & 3016376 & 1.3 & 0.7 & 0.3 & $1.23_{-0.38}^{+0.62}$ & $43.77_{-32.22}^{+54.38}$ & 145.22 & 2.14 & $6.1_{-5.1}^{+39.2}$ & III & -1.81 & -0.92 \\
\hline c2dJ034504.7+321501 & 3001936 & 0.7 & 4.02 & 0.27 & $0.24_{-0.14}^{+0.62}$ & $33.12_{-30.23}^{+61.54}$ & 8.6 & 0.45 & $106.0_{-70.2}^{+443.6}$ & II & -0.04 & 1.44 \\
\hline c2dJ034513.5+322435 & 3018104 & 0.5 & 3.17 & 0.24 & $0.16_{-0.06}^{+0.35}$ & $70.84_{-66.73}^{+26.59}$ & 10.69 & 0.26 & $1.9_{-0.9}^{+7.1}$ & II & -0.54 & -0.07 \\
\hline \multicolumn{13}{|c|}{ IR4 band turnoff } \\
\hline c2dJ032854.1+311654 & 3012867 & 0.7 & 6.86 & 0.24 & $0.86_{-0.7}^{+1.21}$ & $24.61_{-23.57}^{+70.31}$ & 98.6 & 38.52 & $2.4_{-1.4}^{+4.8}$ & II & -0.72 & 0.38 \\
\hline
\end{tabular}




\begin{tabular}{|c|c|c|c|c|c|c|c|c|c|c|c|c|}
\hline $\mathrm{ID}_{\mathrm{c} 2 \mathrm{~d}}$ & $\mathrm{ID}_{\text {model }}$ & $\chi_{r}^{2}$ & $A_{V}$ & $D$ & $M_{\star}$ & $\mathrm{Age}_{\star}$ & $L_{\star}$ & $L_{\text {dust }}$ & $R_{\mathrm{in}} / R_{\mathrm{sub}}$ & Stage & $\alpha_{\text {turnoff }}$ & $\alpha_{\text {excess }}$ \\
\hline (1) & (2) & (3) & $\begin{array}{l}\text { (mag) } \\
\text { (4) }\end{array}$ & $\begin{array}{l}\text { (kpc) } \\
(5)\end{array}$ & $\begin{array}{l}\left(M_{\odot}\right) \\
(6)\end{array}$ & $\begin{array}{l}\left(10^{5} \mathrm{yr}\right) \\
(7)\end{array}$ & $\begin{array}{l}\left(10^{-2} L_{\odot}\right) \\
(8)\end{array}$ & $\begin{array}{l}\left(10^{-2} L_{\odot}\right) \\
(9)\end{array}$ & (10) & (11) & (12) & (13) \\
\hline c2dJ033027.1+302830 & 3009118 & 1.9 & 1.61 & 0.27 & $0.2_{-0.05}^{+0.98}$ & $1.2_{-0.46}^{+6.0}$ & 339.8 & 31.42 & $126.4_{-125.4}^{+76.6}$ & I & 2.76 & 2.76 \\
\hline c2dJ034227.1+314433 & 3007649 & 0.6 & 6.88 & 0.25 & $0.8_{-0.38}^{+0.28}$ & $64.48_{-48.89}^{+30.97}$ & 47.89 & 1.45 & $267.1_{-116.8}^{+24.8}$ & II & 1.17 & 2.1 \\
\hline c2dJ034254.7+314345 & 3009555 & 0.4 & 1.41 & 0.23 & $0.71_{-0.25}^{+0.51}$ & $55.53_{-37.56}^{+43.05}$ & 27.91 & 0.5 & $67.9_{-33.6}^{+92.3}$ & II & 0.13 & 0.93 \\
\hline c2dJ034306.8+314821 & 3012886 & 0.4 & 2.67 & 0.23 & $0.21_{-0.09}^{+0.27}$ & $17.06_{-14.54}^{+20.52}$ & 24.52 & 0.77 & $157.9_{-121.7}^{+794.5}$ & II & 0.2 & 1.17 \\
\hline c2dJ034419.1+320931 & 3010204 & 1.2 & 0.31 & 0.33 & $1.58_{-0.08}^{+0.09}$ & $66.66_{-13.64}^{+32.79}$ & 211.55 & 0.74 & $6.5_{-5.5}^{+5.2}$ & III & -1.84 & -1.45 \\
\hline c2dJ034421.6+321510 & 3009308 & 2.6 & 0.7 & 0.3 & $0.14_{-0.04}^{+0.14}$ & $33.21_{-29.78}^{+22.18}$ & 10.0 & 2.49 & $175.4_{-147.8}^{+2655.6}$ & II & -0.18 & 1.57 \\
\hline c2dJ034431.5+320845 & 3005300 & 2.0 & 1.63 & 0.29 & $1.27_{-0.55}^{+0.58}$ & $26.79_{-11.74}^{+51.42}$ & 186.0 & 37.17 & $194.2_{-42.8}^{+53.9}$ & II & 2.75 & 2.89 \\
\hline c2dJ034456.8+315411 & 3019546 & 0.5 & 2.29 & 0.25 & $0.14_{-0.03}^{+0.1}$ & $79.92_{-24.53}^{+13.93}$ & 4.16 & 0.1 & $49.2_{-48.2}^{+174.8}$ & II & -0.01 & 0.97 \\
\hline c2dJ034507.6+321028 & 3018977 & 0.9 & 0.34 & 0.29 & $1.31_{-0.29}^{+0.4}$ & $38.55_{-27.0}^{+59.59}$ & 215.43 & 1.21 & $3.3_{-2.3}^{+15.0}$ & III & -2.1 & -1.51 \\
\hline \multicolumn{13}{|c|}{ W3 band turnoff } \\
\hline c2dJ032916.7+311618 & 3015991 & 0.7 & 0.8 & 0.22 & $0.27_{-0.11}^{+0.26}$ & $20.12_{-12.41}^{+18.71}$ & 23.09 & 0.04 & $76.1_{-75.1}^{+2504.9}$ & II & -1.55 & 0.86 \\
\hline c2dJ033026.0+310218 & 3018696 & 0.8 & 2.07 & 0.22 & $1.0_{-0.61}^{+0.78}$ & $18.79_{-9.66}^{+65.97}$ & 63.75 & 0.26 & $7.1_{-6.1}^{+1335.9}$ & III & -1.79 & -0.58 \\
\hline c2dJ033351.1+311228 & 3008112 & 1.1 & 2.71 & 0.22 & $0.24_{-0.07}^{+0.08}$ & $8.24_{-4.11}^{+7.82}$ & 26.78 & 1.09 & $795.5_{-752.3}^{+1649.5}$ & II & -0.83 & 0.75 \\
\hline c2dJ034011.8+315523 & 3010915 & 1.7 & 3.57 & 0.25 & $0.51_{-0.15}^{+0.3}$ & $48.64_{-47.47}^{+48.16}$ & 24.27 & 1.29 & $835.7_{-834.7}^{+61.4}$ & II & 4.14 & 8.04 \\
\hline
\end{tabular}

Notes: (1): c2d ID;

(2): ID of the Best-fit model from Robitaille et al. (2006);

(3): Minimum reduced $\chi^{2}$;

(4): The most probable $V$-band interstellar extinction;

(5): The most probable heliocentric distance;

(6): The most probalbe stellar mass and the $95 \%$ confidence interval;

(7): The most probable stellar age and the $95 \%$ confidence interval;

(8): The best-fit stellar bolometric luminosity;

(9): Dust luminosity from integral of the model SED of disk+envelope that best fits the observed SED;

(10): The disk inner radius, in units of the dust sublimation radius, and the $95 \%$ confidence interval;

(11): Evolutionary stage;

(12): Spectral indices at $\lambda>\lambda_{\text {turnoff }}$ for stellar photosphere-included IR SEDs.

(13): Spectral indices at $\lambda>\lambda_{\text {turnoff }}$ for stellar photosphere-subtracted IR SEDs.

Acknowledgements We thank the anonymous referee for his/her helpful comments that improved this manuscript. We acknowledge the support of the National Natural Science Foundation of China grant \#11390373. HXZ acknowledges support from China Postdoctoral Science Foundation under Grant No. 2013M530008, and CAS-CONICYT Postdoctoral Fellowship, administered by the Chinese Academy of Sciences South America Center for Astronomy (CASSACA). MF acknowledges the NSFC under grant 11203081.

\section{References}

Alexander, R. D., 2008, New Astron. Rev., 52, 60

Alexander, R. D., Clarke, C. J., \& Pringle, J. E. 2006a, MNRAS, 369, 216

Alexander, R. D., Clarke, C. J., \& Pringle, J. E. 2006b, MNRAS, 369, 229

Alexander, R. D., Pascucci, I., Andrews, S., Armitage, P., \& Cieza, L. 2014, Protostar and Planets VI, Henrik

Beuther, Ralf S. Klessen, Cornelis P. Dullemond, and Thomas Henning (eds.), University of Arizona Press,

Tucson, 914 pp., p. 475-496

Allen, L. E., et al. 2004, ApJS, 154, 363

Andersen, M., Meyer, M. R., Greissl, J., \& Aversa, A. 2008, ApJL, 683, 183

André, P., \& Ward-Thompson, D., \& Barsony, M. 1993, ApJ, 406, 122

Andrews, S. M., \& Williams, J. P. 2005, ApJ, 631, 1134

Artymowicz, P., \& Lubow, S. H. 1994, ApJ, 421, 651

Backman, D. E., \& Paresce, F. 1993, in Protostars and Planets III, ed. E. H. Levy \& J. I. Lunine (Tucson: Univ. Arizona Press), 1253 
Bally, J., Walawender, J., Johnstone, D., Kirk, H., \& Goodman, A. A. 2008, in Handbook of Star Forming Regions, Vol. 1, ed. Bo Reipurth, 308

Beckwith, S. V. W., Sargent, A. I., Chini, R., \& Gusten, R. 1990, AJ, 99, 924

Belikov, A. N., Kharchenko, N. V., Piskunov, A. E., Schilbach, E., Scholz, R. D. 2002, A\&A, 387, 117

Baraffe, I., Chabrier, G., Allard, F., \& Hauschildt, P. H. 1998, A\&A, 337, 403

Bernasconi, P. A., Maeder, A. 1996, A\&A, 307, 829

Brown, J. M., Blake, G. A., Dullemond, C. P., et al. 2007, ApJ, 664, 107

Chiang, E. I., \& Goldreich, P. 1997, ApJ, 490, 368

Currie, T., \& Kenyon, S. J. 2009, AJ, 138, 703

Currie, T., \& Sicilia-Aguilar, A. 2011, ApJ, 732, 24

Chen, B. -Q., Liu, X. -W., Yuan, H. -B., et al. 2014, MNRAS, 443, 1192

Chen, B. -Q., Liu, X. -W., Yuan, H. -B., Huang, Y., \& Xiang, M. -S. 2015, MNRAS, 448, 2187

Calvet, N., D’Alessio, P., Hartmann, L., et al. 2002, ApJ, 568, 1008

Calvet, N., Briceño, C., Hernández, J., et al. 2005, AJ, 129, 935

Cieza, L., Padgett, D. L., Stapelfeldt, K. R., et al. 2007, ApJ, 667, 308

Cieza, L. A., Schreiber, M. R., Romero, G. A., et al. 2010, ApJ, 712, 925

Cui, X. -Q., Zhao, Y. -H., Chu, Y. -Q., et al. 2012, RAA, 12, 1197

Dahm, S. E., \& Carpenter, J. M. 2009, AJ, 137, 4024

de Zeeuw, P. T., Hoogerwerf, R., de Bruijne, J. H. J., Brown, A. G. A., Blaauw, A. 1999, AJ, 117, 354

Dullemond, C. P., \& Dominik, C. 2005, A\&A, 434, 971

Dullemond, C. P., Hollenbach, D., Kamp, I., \& D’Alessio, P., et al. 2007 Protostars and Planets V, University of Arizona Press (2007), eds. B. Reipurth, D. Jewitt, K. Keil (arXiv0602619)

D’Antona, F., \& Mazzitelli, I. 1997, in Cool stars in Clusters and Associations, ed. R. Pallavicini, \& G. Micela, Mem. S. A. It., 68, 807

Dotter, A., Chaboer, B., Jevremovic, D., et al. 2008, ApJS, 178, 89

Espaillat, C., Ingleby, L., Furlan, E., et al. 2013, ApJ, 762, 62

Evans, II, N. J. et al. 2003, PASP, 115, 965

Evans, II, N. J., Dunham, M. M., J $\phi$ rgensen, J. K., et al. 2009, ApJS, 181, 321

Enoch, M. L., et al. 2006, ApJ, 638, 293

Eiroa, C., Marshall, J. P., Mora, A., et al. 2013, A\&A, 555, 11

Fang, M., van Boekel, R., Wang, W., et al. 2009, A\&A, 504, 461

Fang, M., van Boekel, R., King, R. R., et al. 2012, A\&A, 539, 119

Fang, M., Kim, J. S., van Boekel, R., Sicilia-Aguilar, A., Henning, T., \& Flaherty, K., 2013, ApJS, 207, 5

Fitzpatrick, E. L. 1999, PASP, 111, 63

Greissl, J., Meyer, M. R., Wilking, B. A., Fanetti, T., Schneider, G., Greene, T. P., \& Young, E. 2007, AJ, 133, 1321

Greene, T. P., Wilking, B. A., André, P., Young, E. T., \& Lada, C. J. 1994, ApJ, 434, 614

Gori, U., \& Hollenbach, D. 2009, ApJ, 690, 1539

Goodman, A. A., Alves, J. F., Arce, H. G., et al. 2005, Bulletin of the American Astronomical Society, Vol. 37, p. 1475

Hartmann, L., Calvet, N., Gullbring, E., D’Alessio, P. 1998, ApJ, 385

Hsieh, T. -H., \& Lai, S. -P., 2013, ApJS, 205, 5 (HL13)

Hatchell, J., Richer, J. S., Fuller, G. A., Qualtrough, C. J., Ladd, E. F., \& Chandler, C. J. 2005, A\&A, 440, 151

Hernández, J., Hartmann, L., Megeath, T., et al. 2007, ApJ, 662, 1067

Hillenbrand, L. A., \& Carpenter, J. M. 2000, ApJ, 540, 236 
Hillenbrand, L. A., Bauermeister, A., \& White, R. J. 2008, in ASP Conf. Ser. 384, 14th Cambridge Workshop on

Cool Stars, Stellar Systems, and the Sun, ed. G. van Belle (San Francisco, CA: ASP), 200

Hillenbrand, L. A., \& Carpenter, J. M. 2000, ApJ, 540, 236

Harvey, P., Merín, B., Huard, T. L., et al. 2007, ApJ, 663, 1149

Henning, T., \& Meeus, G. 2011, Dust Processing and Mineralogy in Protoplanetary Accretion Disks, ed. P. J. V.

Garcia (Chicago: University of Chicago Press), 114

Herbig, G. H. 1998, ApJ, 497, 736

Hernández, J., Calvet, N., Briceño, C., et al. 2007, ApJ, 671, 1784

Hughes, A. M., Andrews, S. M., Wilner, D. J., et al. 2010, AJ, 140, 887

Hollenbach, D., Johnstone, D., Lizano, S., Shu, F. 1994, ApJ, 428, 654

Howard, C. D., Sandell, G., Vacca, W. D., et al. 2013, ApJ, 776, 21

J $\phi$ rgensen, J. K., Johnstone, D., Kirk, H., \& Myers, P. C. 2007, ApJ, 656, 293

Kenyon, S. J, Hartmann, L., 1987, ApJ, 323, 714

Kenyon, S. J., \& Hartmann, L. 1995, ApJS, 101, 117

Kim, K. H., Watson, D. M., Manoj, P., et al. 2009, ApJ, 700, 1017

Kley, W., \& Nelson, R. P. 2012, ARA\&A, 50, 211

Keane, J. T., Pascucci, I., Espaillat, C., et al. 2014, ApJ, 787, 153

Lubow, S. H., \& D’Angelo, G. 2006, ApJ, 641, 526

Lupton, R. 2005, http://www.sdss.org/dr5/algorithms/sdssUBVRITransform.html

Luhman, K. L., Rieke, G. H., Young, E. T., Cotera, A. S., Chen, H., Rieke, M. J., Schneider, G., \& Thompson, R. I. 2000, ApJ, 540, 1016

Luhman, K. L., Briceño, C., Stauffer, J. R., Hartmann, L., Barrado y Navascués, D., \& Caldwell, N. 2003a, ApJ, 590,348

Luhman, K. L., Stauffer, J. R., Muench, A. A. et al. 2003b, ApJ, 593, 1093

Luhman, K. L., Allen, P. R., Espaillat, C., Hartmann, L., \& Calvet, N. 2010, ApJS, 186, 111

Luhman, K. L. 2007, ApJS, 173, 104

Lucas, P. W., Roche, P. F., \& Tamura, M. 2005, MNRAS, 361, 211

Lada, C. J., Muench, A. A., Luhman, K. L., et al. 2006, 131, 1574

Lada, C. J. 1987, in Star Forming Regions, ed. M. Peimbert \& J. Jugaku (Dordrecht: Reidel), 1

Levine, J. L., Steinhauer, A., Elston, R. J., \& Lada, E. A. 2006, ApJ, 646, 1215

Lynden-Bell, D., \& Pringle, J. E. 1974, MNRAS, 168, 603

Liu, X. -W., Yuan, H. -B., Huo, Z. -Y., et al. 2014, Proceedings of the International Astronomical Union, IAU Symposium, 298, 310

Merín, B., J $\phi$ rgensen, J., Spezzi, L. et al. 2008, ApJS, 177, 551

Merín, B., Brown, J. M., Oliveira, I., et al. 2010, ApJ, 718, 1200

Moraux, E., Bouvier, J., Stauffer, J. R., \& Cuillandre, J. -C. 2003, A\&A, 400, 891

Morishima, R. 2012, MNRAS, 420, 2851

Muench, A. A., Lada, E. A., Lada, C. J., \& Alves, J. 2002, ApJ, 573, 366

Muench, A. A., Lada, E. A., Lada, C. J., et al. 2003, AJ, 125, 2029

Muench, A. A., Lada, C. J., Luhman, K. L., Muzerolle, J., \& Young, E. 2007, AJ, 134, 411

Muzerolle, J., Calvet, N., Hartmann, L., \& D’Alessio, P. 2003, ApJ, 597, 149

Muzerolle, J., Adame, L., D’Alessio, P., et al. 2006, ApJ, 643, 1003

Matthews, B. C., Kennedy, G., Sibthorpe, B., et al. 2014, ApJ, 780, 97

Najita, J. R., Strom, S. E., \& Muzerolle, J. 2007, MNRAS, 378, 369

Owen, J. E., Ercolano, B., Clarke, C. J., Alexander, R. D. 2010, MNRAS, 401, 1415 
Owen, J. E., \& Jackson, A. P., 2012, MNRAS, 425, 2931

Palla, F., \& Stahler, S. W. 1999, ApJ, 525, 772

Povich, M. S., Kuhn, M. A., Getman, K. V., et al. 2013, ApJS, 209, 31

Ridge, N. A., Di Francesco, J., Kirk, H., et al. 2006, AJ, 131, 2921

Robitaille, T. P., Whitney, B. A., Indebetouw, R., Wood, K., \& Denzmore, P. 2006, ApJS, 167, 256

Robitaille, T. P., Whitney, B. A., Indebetouw, R., \& Wood, K. 2007, ApJS, 169, 328

Robitaille, T. P., 2008, in Beuther H., Linz H., Henning T., eds, ASPConf. Ser. Vol. 387. Massive Star Formation:

Observations Confront Theory. SED Modeling of Young Massive Stars. ASP, San Francisco, p. 290

Roeser, S., Demleitner, M., Schilbach, E. 2010, AJ, 139, 2440

Rice, W. K. M., Wood, K., Armitage, P. J., et al. 2003, MNRAS, 342, 79

Salpeter, E. E. 1955, ApJ, 121, 161

Shu, F. H. et al. 1993, Icarus, 106, 92

Slesnick, C. L., Hillenbrand, L. A., \& Carpenter, J. M. 2004, ApJ, 610, 1045

Shakura, N. I., \& Sunyaev, R. A. 1973, A\&A, 24, 337

Scholz, A., Geers, V., Jayawardhana, R., Fissel, L., Lee, E., Lafreniere, D., \& Tamura, M. 2009, ApJ, 702, 805

Surace, J. A., Sanders, D. B., Mazzarella, J. M. 2004, AJ, 127, 3235

Strom, S. E., Strom, K. A., Carrasco, L. 1974, PASP, 86, 798

Swenson, F. J., Faulkner, J., Rogers, F. J., \& Iglesias, C. A., 1994, ApJ, 425, 286

Siess, L., Dufour, E., \& Forestini, M. 2000, A\&A, 358, 593

Sicilia-Aguilar, A., Hartmann, L. W., Calvet, N., et al. 2006, ApJ, 638, 897

Sicilia-Aguilar, A., Roccatagliata, V., Getman, K., et al. 2015, A\&A, 573, 19

Strom, K. M., Strom, S. E., Edwards, S., Cabrit, S., \& Skrutskie, M. F. et al. 1989, AJ, 97, 1451

Skrutskie, M. F., Dutkevitch, D., Strom, S. E., et al. 1990, AJ, 99, 1187

Su, K. Y. L., Rieke, G. H., Stansberry, J. A., et al. 2006, ApJ, 653, 675

Tanaka, H., Himeno, Y., \& Ida, S. 2005, ApJ, 625, 414

Trilling, D. E., Bryden, G., Beichman, C. A., et al. 2008, ApJ, 674, 1086

Weights, D. J., Lucas, P. W., Roche, P. F., Pinfield, D. J., \& Riddick, F. 2009, MNRAS, 392, 817

Wilking, B. A., Meyer, M. R., Greene, T. P., Mikhail, A., \& Carlson, G. 2004, AJ, 127, 1131

Winston, E., Megeath, S. T., Wolk, S. J., et al. 2009, AJ, 137, 4777

Winston, E., Megeath, S. T., Wolk, S. J., et al. 2010, AJ, 140, 266

Williams, J. P., \& Cieza, L. A. 2011, ARA\&A, 49, 67

Yi, S., Kim, Y. -C., \& Demarque, P. 2003, ApJS, 144, 259

Yuan, H. -B., et al. 2015, in preparation

Zhang, H.-H., Liu, X.-W., Yuan, H.-B., et al., 2013, Research in Astronomy and Astrophysics, 13, 490

Zhang, H.-H., Liu, X.-W., Yuan, H.-B., et al. 2014, Research in Astronomy and Astrophysics, 14, 456

Zhu, Z., Nelson, R. P., Dong, R. et al. 2012, ApJ, 755, 6

This paper was prepared with the RAA LATEX macro v1.2. 